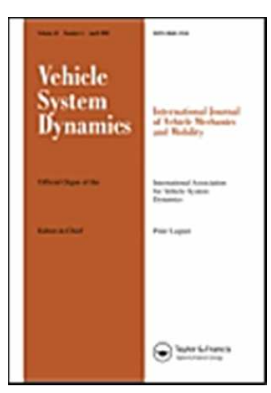

\title{
Automated Driving and Autonomous Functions on Road Vehicles
}

\begin{tabular}{|r|l|}
\hline Journal: & Vehicle System Dynamics \\
\hline Manuscript ID: & NVSD-2015-0054.R1 \\
\hline Manuscript Type: & Special Issue Paper \\
\hline Date Submitted by the Author: & n/a \\
\hline Complete List of Authors: & $\begin{array}{l}\text { Gordon, Timothy; University of Lincoln, } \\
\text { Lidberg, Mathias; Chalmers University of Technology, Applied Mechanics }\end{array}$ \\
\hline Keywords: & Active safety, Autonomous vehicles, Control \\
\hline & \\
\hline
\end{tabular}

\section{SCHOLARONE ${ }^{\text {Im }}$}

Manuscripts 
To appear in Vehicle System Dynamics Vol. 00, No. 00, Month 20XX, 1-38

\title{
Automated Driving and Autonomous Functions on Road Vehicles
}

\author{
T.J. Gordon ${ }^{a *}$, M. Lidberg ${ }^{b}$ \\ ${ }^{\mathrm{a}}$ School of Engineering, University of Lincoln, LN6 7TS, Lincoln, UK; ${ }^{\mathrm{b}}$ Department of Applied \\ Mechanics, Chalmers University of Technology, SE-412 96, Gothenburg, Sweden \\ (Received day-month-year.)
}

\begin{abstract}
In recent years, road vehicle automation has become an important and popular topic for research and development in both academic and industrial spheres. New developments received extensive coverage in the popular press, and it may be said that the topic has captured the public imagination. Indeed, the topic has generated interest across a wide range of academic, industry and governmental communities, well beyond vehicle engineering; these include computer science, transportation, urban planning, legal, social science and psychology. While this follows a similar surge of interest - and subsequent hiatus - of Automated Highway Systems in the 1990's, the current level of interest is substantially greater, and current expectations are high. It is common to frame the new technologies under the banner of "self-driving cars" - robotic systems potentially taking over the entire role of the human driver, a capability that does not fully exist at present. However, this single vision leads one to ignore the existing range of automated systems that are both feasible and useful. Recent developments are underpinned by substantial and long-term trends in "computerisation" of the automobile, with developments in sensors, actuators and control technologies to spur the new developments in both industry and academia. In this paper we review the evolution of the intelligent vehicle and the supporting technologies with a focus on the progress and key challenges for vehicle system dynamics. A number of relevant themes around driving automation are explored in this article, with special focus on those most relevant to the underlying vehicle system dynamics. One conclusion is that increased precision is needed in sensing and controlling vehicle motions, a trend that can mimic that of the aerospace industry, and similarly benefit from increased use of redundant by-wire actuators.
\end{abstract}

Keywords: self-driving cars, vehicle automation, intelligent control, active safety, collision avoidance, vehicle sensors, driver-vehicle interaction.

\section{Introduction}

In the past, cars and trucks could be considered as complex mechanical systems, but since the 1980's they have become increasingly sophisticated mechatronic systems, fitted with many actuators, sensors and electronic control units. From early beginnings with fuel control, the level and authority of electronic control systems on vehicles has become increasingly sophisticated, including automated braking systems which operate independently of the driver to avoid collisions, automated steering for parking (capable of manoeuvring into smaller parking spaces than the average human driver), and automated driving (speed and steering) systems for use at low speed in traffic jams.

\footnotetext{
*Corresponding author. Email: tgordon@lincoln.ac.uk
} 
Several companies including Google, Volvo, Tesla and Daimler have been demonstrating fully self-driving vehicles that use computers rather than humans to do the driving. For safety reasons, these driverless or autonomous vehicles have test engineers closely supervising their progress. There are, however, intentions to make self-driving cars available to the general public. The US states of Nevada and California have introduced legislation to allow testing of self-driving cars on their highways, and the UK and German government recently announced similar initiatives.

Motivation for these developments are centred around a broad "technological fix" agenda - expanding human mobility, increasing travel efficiency in terms of time and fuel, freeing up the connected traveller from the demands of routine driving, and potentially improving safety. It is common to read statements like "over $90 \%$ of highway accidents occur due to driver-related human errors" [1], but we should not fall into the trap of concluding that automation implies a safety benefit - in the future we could be saying "over $90 \%$ of highway accidents are due to computer error".

Changing the way we drive road vehicles in a fundamental way offers many opportunities, and in this paper we explore the intersection of recent developments in this field with the fundamental engineering perspectives of vehicle system dynamics. In some scenarios the whole framework of road vehicle dynamics may change, so in the following we will evaluate these developments in the context of a long line of technological advances, much of which has arisen from or been influenced by the research outputs of the International Association of Vehicle System Dynamics.

Ceding complete control of cars to computers is just one end of a spectrum of automation concepts for road vehicles; there exists a growing portfolio of more limited but useful capabilities, particularly in areas of driver assistance and active safety.

An early example of the technologies needed for self-driving vehicles is the antilock braking system (ABS) [2]. Developed in the 1980s, ABS technology monitors wheel speeds and controls brake pressures to prevent wheels from locking up under hard braking. ABS may not be considered particularly smart these days, but its sensors and actuators provided a gateway towards more sophisticated systems. For example, in electronic stability control (ESC) [3], [4], an electronic control unit intervenes by using the brakes to correct for loss of stability, a significant risk on wet or icy roads. Compared with ABS, ESC interacts with the driver more conspicuously, correcting the way the car moves via feedback control: ESC compares actual with expected vehicle motions, and prevents the car from spinning dangerously or slipping sideways by the application of correcting yaw moments. Taken together, ESC and electronic engine control make it relatively simple and affordable to use engine and brakes to control a vehicle's speed, which is a suitable starting point for at least partially automating the driving process.

The above "ABS perspective" represents a particular 'entry route' to vehicle automation, focused around the traditional automotive companies and suppliers, building generation-by-generation on new actuators, sensors and interfaces. An alternative route has emerged from two competitive challenges, organized by the Defense Advanced Research Projects Agency (DARPA) in the United States during 2004-2007 [5],[6],[7]. The DARPA Grand Challenge involved autonomous driving across desert tracks (car vs. terrain) while the subsequent DARPA Urban Challenge introduced traffic conflicts via a simulated city scenario. While sensing and mapping capabilities in these challenges were far from what is economically feasible with current production cars, they do illuminate what is possible with current technologies when production costs are ignored. And, unlike the ABS/ESC developments, the focus was on computation and information processing, more than on the underlying vehicle dynamics and control systems.

Hence, from this 'DARPA perspective', the interesting challenges are mostly related to 


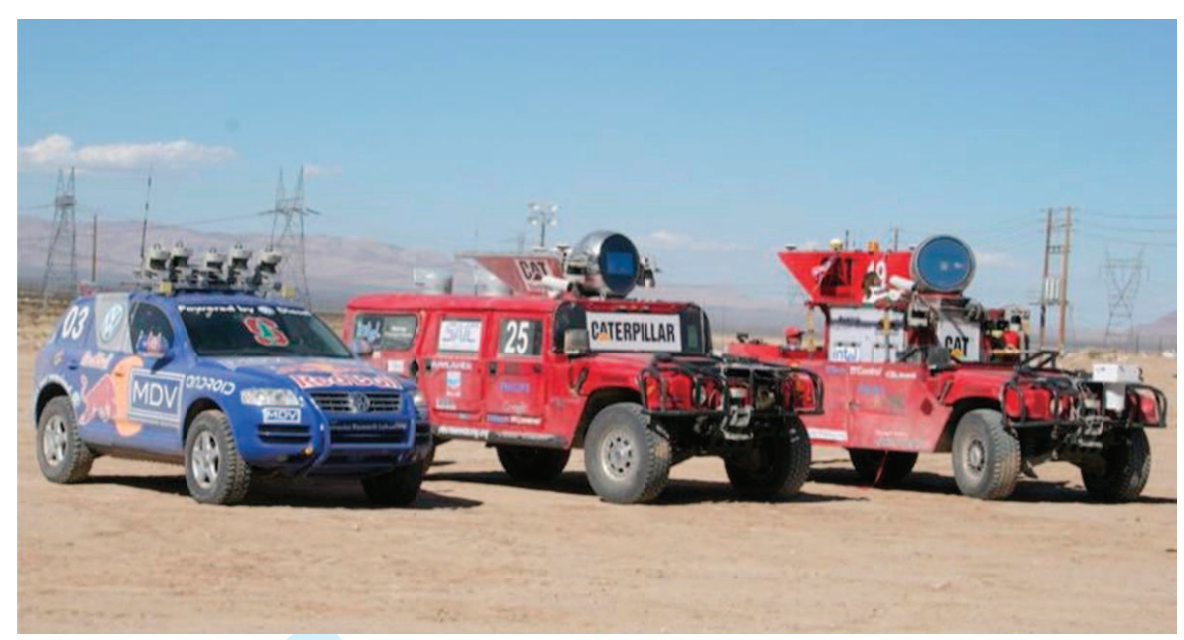

Figure 1. The top three finishers in the 2005 DARPA Grand Challenge; vehicles from Stanford University (left), and Carnegie Mellon University (centre and right). Reproduced from [7].

sophisticated optical systems and image processing, high powered computation, precision location and mapping, data fusion and (artificial) intelligence. These capabilities, so clearly an existing part of what the human driver achieves, occupy the "tactical space" of driving. This is part of a well-established conceptual model of human driving that is worthy of closer inspection given the new trends in vehicle automation. The well-accepted conceptual model of Michon [8] states that there are three levels of human activity in traditional manual driving: strategic (planning), tactical (maneuvering and shortterm decision making) and control (actuation of steering etc. typically using localised feedback).

In Figure 2, we formulate that the Strategic, Tactical and Control processes may involve automated systems, in full or in part. Note that there are different timescales of operation between these levels, ranging from minutes/hours/days at the strategic level, to a few seconds for maneuvering, and then down to a few tens of milliseconds at the control level [8]. These timings are inherited from the needs of vehicle motion control itself, so there is little reason to expect them to change significantly as a result of automation.

At the upper level, strategic decisions affect the general planning stage of a trip, including goals, route, and possibly choice of transportation mode, based on preferences, costs and perceived risks. General considerations about transport and mobility may influence these decisions, as indeed may information gathered before or during a trip. In the modern "connected" world, with near-universal access to real-time information, it is common for general plans to evolve over time. In Figure 2 the Environmental Inputs are assumed to be directly perceived (e.g. weather conditions, visually) while External processes are considered to be the result of separate agencies, e.g. public information services. Note: we use the term driver throughout, meaning the person in the vehicle most likely to carry out actions in response to some event. For discussion, we exclude the case of a vehicle without any human occupant.

The Tactical Level is where the driver and/or automated systems plan manoeuvres within traffic, decide to change lanes, slow for a curve, prepare to take a motorway exit lane etc. In Figure 2 these actions - plus supporting information processing - are represented by Tactical Level block. Again, directly relevant information comes from the left (road geometry, traffic movements etc.) while agency-sourced information comes from the right. According to [8] the actions are in the form of "controlled action patterns" from 


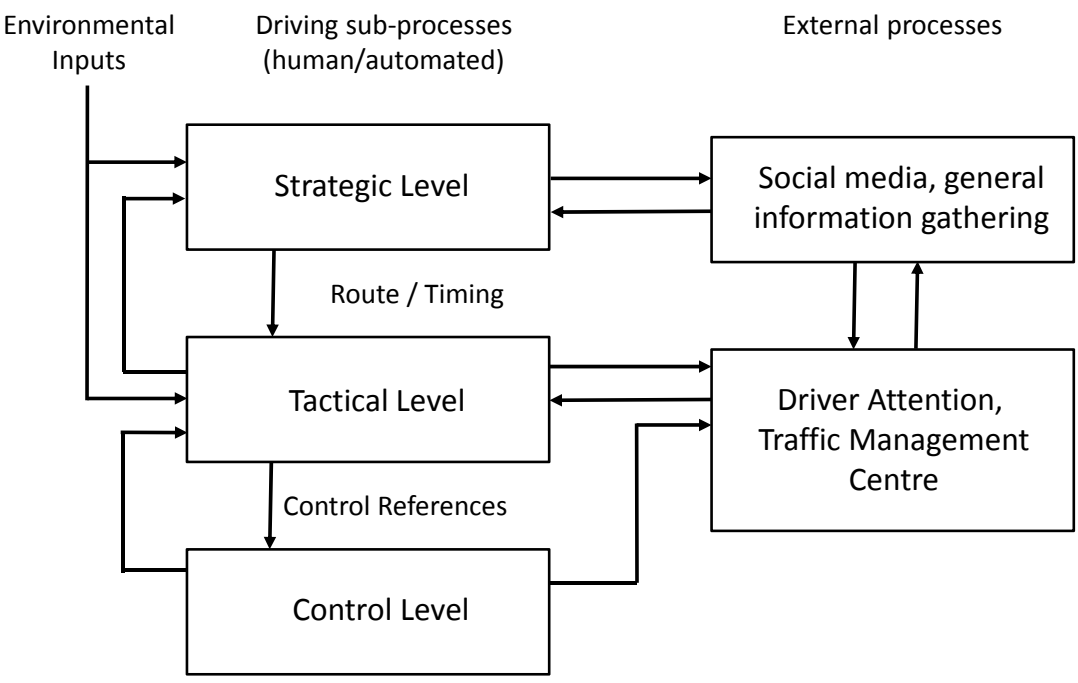

Figure 2. Hierarchical structure of the driving task including attention switching (after Michon [8]).

the driver, implying that conscious decisions or plans are made based on the evolving road and traffic situation, or that previous experience is used to decide the timing and amplitude of lower level control actions. For (partially or fully) automated systems, this layer may for example determine feasible or desirable vehicle trajectories that can then be passed to the control level.

Here we assume that the tactical level block is purely concerned with actual driving, so to represent the common behaviour of attention switching to non-driving activities (a typical and serious safety concern [9], [10], [11]) this is shown as a secondary process of attention control - visual or cognitive or both. While such attention switching is already considered important in the safety of normal manual driving, it is worth further emphasising its role in the case of partial or fully automated driving, as it does influence the availability of the driver to participate in driving actions.

As well as human driver attention, we allow for the activities of external agencies, for example a traffic management centre which monitors traffic conditions and provides driving-relevant information to the human driver and/or any automated systems. And it may be that such information directly influences the reference information being passed down to the control level (e.g. ice warnings influence the action threshold for a collision avoidance system). As mentioned, from the "DARPA perspective" the major developments have been rooted in the tactical level.

The control level responds to reference information coming from the tactical block, so rather than "seeing" lane markers the control layer would "see" the need to increase yaw rate or decrease speed according to some objective reference information. The dividing line between the tactical and control levels is not universal and may depend on the algorithms used; for example an Adaptive Cruise Control system (ACC: tactical level) might determine a target speed as reference for a simple classical feedback controller, while in collision avoidance the tactical level system may define a set of motion boundaries for a Model Predictive Control (MPC: control level) to use in the integrated control of steering and brakes. The separation is clearest when looking at the information processing demands; the tactical level must sense and interpret the driving environment, while 
the control level is required only to give best available performance based on objective information supplied. The upward arrow, from control to tactical (also from tactical to strategic) are included to indicate pathways for adaptation. Of course there may be other information flows, for example if a brake controller directly sends friction information to a traffic control centre, but Figure 2 is intended to show the major pathways. Note that from the "ABS perspective" mentioned above, most significant developments have emerged from the control level, albeit with significant intrusions into the tactical level.

The focus of this paper is to frame concepts and review progress and challenges in the area of vehicle and driving automation which have particular implications for the community of vehicle dynamics and control engineers. This is also intended to move discussion away from an existing narrow and perhaps naive view that to replace the driver in a vehicle simply requires the removal all human elements from the three levels in Figure 2, achieving this via expanded sensor sets and information processing by artificial intelligence algorithms. It is born of the belief that what is already known about tyre mechanics, vehicle stability, direct yaw moment control, friction estimation, driveline control, brake system performance, driver modelling etc. etc. are important to the development of the new integrated technologies and will crucially influence their performance and safety.

This defines a convenient starting point for the main thrust of this review paper, which is organized as follows. In Section 2 we review some key developments that have led to the current generation of autonomous functions on road vehicles, with focus on those systems which first ventured into the tactical level of driving. Then in section Section 3 we review another categorization of "levels" of driving automation, and based on this we consider the recent progress made at both the lower and higher levels. For intelligent vehicles to seriously enter the tactical driving domain requires high degree precision sensing of the environment; in Section 4 the issues of sensing and localization relative to maps - or directly relative to road boundaries or threats etc. - is considered. In Section 5 we review progress in establishing the safety of automated driving systems, noting that some raise safety concerns via possible unintended consequences, while others are specifically designed to protect drivers and others, e.g. via collision warning and prevention. Automation offer potential for precise motion control, and in Section 6 we review progress and opportunities for using optimization and optimal control techniques for benefits in energy, transport efficiency, individual time-saving (mainly at the large-scale). Another design concept, away from optimal driving, is naturalistic driving aimed at creating automated driving systems that are 'less robotic' and perhaps more acceptable to people - Section 7. Then in Section 8 the focus is on the automation of limit handling, with applications for collision avoidance and other near-crash active safety systems. Finally, in Section 9 we focus on what is feasible and desirable for increased automation at all levels, including migrating towards full driving automation in the future. In particular we focus on which opportunities and challenges are most significant for researchers in vehicle dynamics and control.

\section{Evolution of the Intelligent Vehicle}

As described above, an initial electronic control evolution took place in the area of ABS and ESC for chassis systems; prior to this was the development of Electronic Engine Control (electronic fuel and mixture control, replacing the mechanical carburetor) which led to the further development of vehicle speed regulation in conventional cruise control. However, with no relevant environmental sensing in these systems, they all sit at the lower 
control level of driving of Figure 2. To contribute to the tactical driving the "Intelligent Vehicle" is directly aware of the driving environment and responds directly to it.

\subsection{Automated Speed Control}

Conventional Cruise Control (CCC) are popular features on cars, particular in countries where the roads are longer and straighter and destinations are farther apart, such as Australia and North America. CCC allows drivers to set a maximum speed at which they want to travel and the system controls the throttle and maintains the speed of the vehicles even on steep gradients. Despite the popularity of CCC there are relatively few studies that have examined the safety benefit/disbenefits and acceptance by drivers [12], [13]. By adding a forward-pointing radar, a vehicle gains sufficient environmental information - range and range-rate of the preceding vehicle [14] - to adapt to traffic by reducing speed when there is a slower vehicle in front, otherwise tracking the driverselected set speed. This provided an early step into automating aspects of tactical driving in the form of adaptive cruise control systems (ACC). ACC was introduced in the market in 1995 by Mitsubishi in Japan [15] and rapidly gained acceptance. Today a growing list of car manufacturers offer ACC, including Cadillac, Jaguar, Honda, BMW and Volvo. For a more detailed description of ACC, including many references, see [16] and [17]. A number of studies have shown the trend of ACC driving to reduce tailgating as well as reducing workload for the driver. Early research was conducted at the University of Michigan and reported in [18] and similar findings were reported in [19]. On the other hand there is the potential for over-reliance on the automated ACC system, some authors have suggested potential pitfalls, for example due to misunderstanding of the ACC functionality leading to an increased collision risk [20], and also due to disengagement from the driving task leading to an increased response time [21],[13] as illustrated in Figure 3.
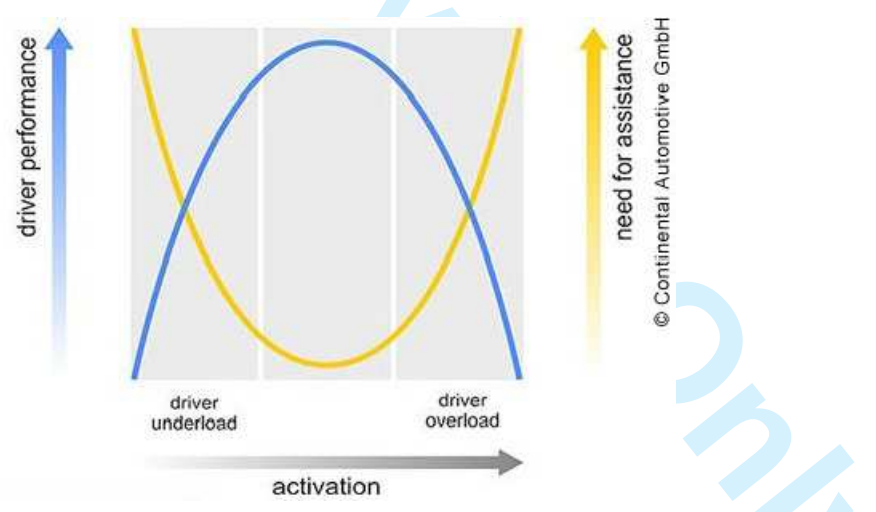

Figure 3. Driver performance and need for assistance depends on the activation of the driver (Picture: HAVEit project, Continental Automotive $\mathrm{GmbH}$ ).

While ACC is a comfort system, designed to assist the driver and reduce workload, it does directly relate to a form of conflict and crash i.e. forward collision. It is not directly designed to deal with such conflicts; if the car in front brakes suddenly and beyond the authority of the ACC, there is a warning issued and the driver is expected to take over speed control. Hence there exists a corresponding opportunity for accident prevention or mitigation in the form of Autonomous (or Automatic) Emergency Braking (AEB) $([22],[23])$ Here the sensor and actuator requirements are essentially the same as for ACC, but the control algorithms are very different and control authority is increased: system 
activation occurs without any driver command, and high levels of deceleration result from that activation. Thus, as a result of the technical opportunity, collision avoidance and crash mitigation systems came onto the market as further developments of automatic speed control. In these systems, the car, or lorry, automatically applies its brakes to avoid a crash or reduce the severity of an anticipated impact. This is another example of existing actuators and sensors from ABS and ACC being co-opted for a new purpose, with the vehicles computer given authority to apply the brakes even when there is no command from the driver. The systems role is elevated from reducing the drivers workload to preventing crashes ([24]). In fact, it is part of the evolving new generation of autonomous safety functions in passenger cars and heavy vehicles.

\subsection{Automated and Shared Steering Control}

There is equal opportunity for automating directional control. Usually directional control comes from turning the steering wheel. Typical demonstrations of self-driving show the steering wheel moving by itself. As with speed automation, the key requirement is to sense the cars motion relative to the road, and/or relative to other road users, and then to apply path corrections to reduce or remove errors between the actual and intended paths. Since it is usual though not universal to have a direct mechanical connection between the steering wheel and the direction of the front wheels, there is a design challenge to best assist the driver with steering. One option is to use an Electric Power Assistance System (EPAS) to adjust the steering. This is already common in the power steering of more recent vehicles, where an electric motor replaces the hydraulics. When used in a more interactive or intelligent manner, the car will appear to want to steer of its own accord. Clearly, with hands on the wheel, the driver will perceive the car as having its own intention. Provided drivers are sympathetic to that intention, they will likely allow the steering action. EPAS is already used in self-parking systems where the steering action is fully automated - drivers remove their hands and simply control brake and accelerator during parallel parking. On the other hand, should unexpected steering torque be experienced during regular driving, this could disturb the driver and cause them to resist, which could have dangerous consequences.

There are other options for the car to control its direction, with different implications for the interaction with the driver. For example, with active front steer, an electric motor controls an offset between the steering wheel and the front road wheels [25]. This corrects the steering on the road without creating a corresponding movement of the steering wheel.

In another approach to automated steering, steer-by-wire removes the mechanical connection between the steering wheel and road wheels and so offers further scope to modify the interaction with the driver [26] (Figure 4). This type of electronic control can be used to steer the rear wheels as well as the front, giving more freedom to designers. Yet another option for directional control is to create a left/right bias in the brakes or driveline [27], [28], [29]; for example, by driving the right wheels harder than the left, or braking the left wheels more than the right, the net effect is to turn the vehicle to the left. All the approaches mentioned have their merits and implications in terms of cost, complexity, effect on the vehicles motion and, perhaps most importantly, their synergy with the human driver.

Whatever the mechanism, it is clear that sensors and actuators can provide an automated steering function. As with speed control, the system can function in a self-steering mode only if there is a reference direction/path to follow. This direction can be determined either by the vehicle alone, acting in autonomous mode, or, working in sharedcontrol mode, as an adjustment to what the driver does with the steering wheel. In either 


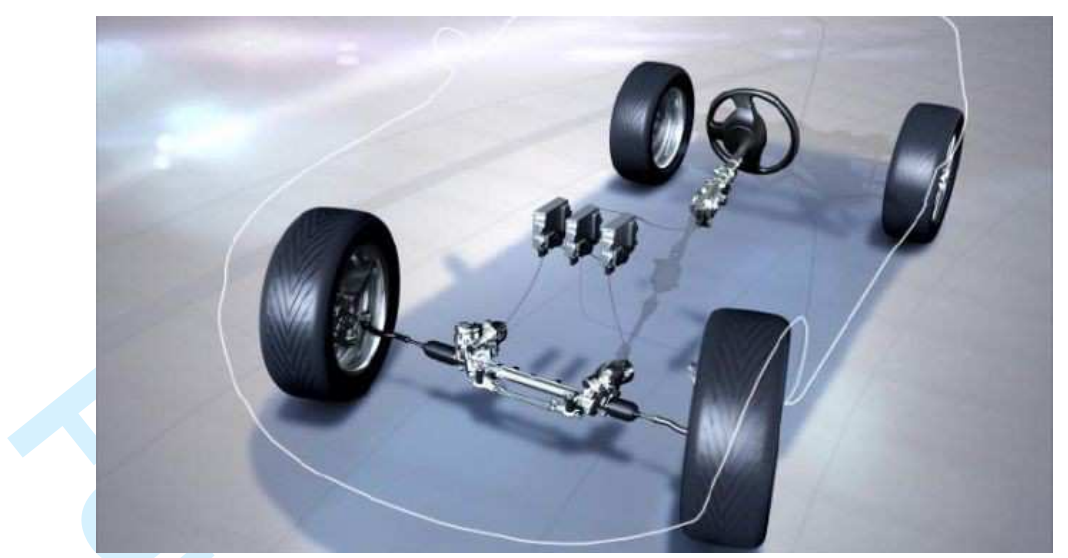

Figure 4. Steer-by-wire system with redundant electronic control units, a backup clutch system that would connect the steering wheel to the tires mechanically if a power failure occur (Picture: Nissan Motors).

case, the vehicle needs to know its position and motion relative to some map of the road and potentially to obstacles, including other vehicles.

In one form of shared steering control, lane-keeping assist or lane-keeping aid (LKA), now available in cars such as the Volvo V60, a camera determines the cars position relative to the lane markings. The electronic steering function then keeps the car in the lane [30]. The steering control is gentle enough to allow the driver to override the system for lane changing or to avoid an obstacle, in much the same way that ACC will defer to the driver for braking or acceleration [31]. In both cases, the responsibility for safety and legal liability stays with the driver.

LKA may also monitor whether the driver is holding the steering wheel. If the drivers remove their hands for more than a short time, the system will give a warning and then disconnect itself. Automated steering can also be a safety enhancement. For example, side-pointing radar can detect another vehicle in the blind spot in an adjacent lane so that the system will resist the drivers steering action if the driver starts to make an unsafe lane change. It is currently difficult to predict the actual safety benefits of such systems [32], even using so-called field operational tests.

\subsection{Semi-Autonomous Driving Systems}

We have seen how the intelligent vehicle with ACC and LKA has emerged from the initial electronic evolution of chassis and powertrain systems. A combination of ACC and LKA could potentially give us an autonomous driving system. However, both systems have limited capabilities: neither is designed to take full authority from the driver. It follows that we still require the driver to supervise: feet off the pedals, hands off the wheel, but eyes firmly on the road. And, importantly, the driver/supervisor should be mentally alert and primed and ready to take control at any instant. Several companies are developing such semi-autonomous systems with the motivation of further reducing the drivers workload. However, the shortfalls of boredom and distraction during a lowlevel supervision task are well known which is why lifeguards at swimming pools are rotated after a short time of supervision.

When the driver has to remain vigilant one doubts there is much genuine workload reduction. On the positive side, the driver can relax their arms and let the steering wheel move by itself. On the negative side, there is the new task of supervising and monitoring 
the automated system, and deciding if and when to take back control. The gain is a reduced physical demand, while the new burden is largely cognitive. Crucially, the semiautonomous vehicle does not alleviate the normal task of anticipating traffic hazards. Added to this are the dangers of overreliance on the system and being distracted by other tasks, potentially leading to new crash risks. This acts as an incentive for creating forever more ingenious systems which reduce crash risk, while at the same time allowing eyes off the road driving.

\section{Levels of Automation}

The large interest in on-road vehicle automation from a wide range of academic, industry and governmental communities has raised the need for a common terminology with a taxonomy and definitions. The new standard J3016 from SAE International simplify communication and facilitate collaboration within technical and policy domains [33]. According to the standard, the levels of driving automation can be divided into Conditional, High and Full Automation, see Figure 5. The standard does not provide complete definitions applicable to lower levels of automation (No Automation, Assisted, or Partial Automation). Active safety and driver assistance system that intervene to avoid and/or mitigate an emergency situation and then immediately disengage are also not included for the various levels of automation.

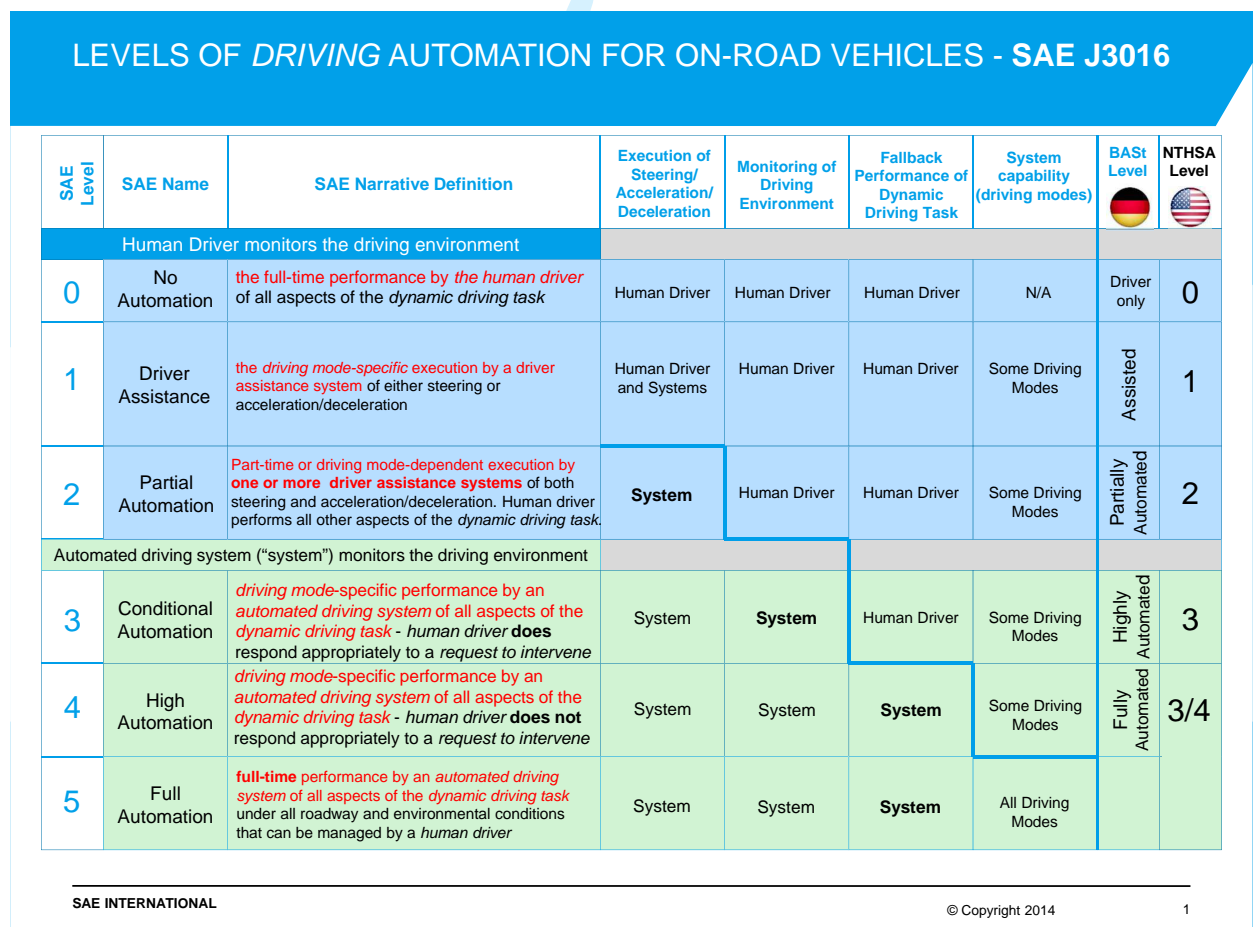

Figure 5. Summary of levels of driving automation for on-road vehicles (after SAE International [33]). 
Considering the level of driving, the human driver continues to perform tactical observation and planning for lower levels of automation. For example, using ACC, the driver is assisted with automated speed control but with steering still under the control of the driver, he or she needs to monitor all aspects of traffic dynamics and road geometry, including being alert to hazards. Going from Partial to Conditional Automation a transition time of several seconds is assumed, during which control can be handed back to the human driver; therefore aspects of tactical driving are shifted from the driver to the automated driving systems. For High Automation the capability is assumed for the automated systems to transition to a minimal risk condition in all situations, so the driver is no longer involved in tactical driving for modes that are automated in this way.

For Conditional Automation (Level 3) the system is expected to perform all modespecific aspects of the dynamic driving task with the expectation that the human driver will respond appropriately to a request to intervene. In cases where immediate release of the driving by the automated driving system could compromise vehicle performance, the system would be expected to delay its release - i.e. gradually relinquish control to ensure a smooth transition. For example, if the vehicle is engaged in a tight turning maneuver, the automated driving system should not release steering control instantaneously, but instead do so gradually as the driver indicates through steering input that he or she is fully re-engaged [33]. Needless to say, this puts new and high requirements on the driver interface e.g. the steering system, as well as requiring sufficient machine intelligence to manage safety critical situations for several seconds at a time. As pointed out earlier, it cannot be assumed that reducing the human drivers involvement in the dynamic driving tasks will necessarily reduce workload. This depends on the level of confidence the driver gives the system, and whether is always works seamlessly and reliably. The system also needs confirmation that the driver is sufficiently alert to recover control, so there may be a workload overhead for the driver in supervising, and an extra demand on the system to monitor and possibly alert the driver to stay engaged.

In the following we focus mainly on demands and current progress around Highly Automated Driving. However we also briefly consider how conventional aspects of vehicle control, even at Level 0, make an effective contribution to the state of the art for driving automation.

\subsection{Conventional Automation}

A review of the conventional control problems of road vehicles - in particular the optimal control of vehicles and systems - was carried out by Sharp and Peng [34]. The major areas were deemed to be as follows, although the authors did state it may not be complete: (a) active and semi-active suspensions; (b) worst-case manoeuvring; (c) minimum time manoeuvring; (d) driver control modeling; (e) state estimation; (f) obstacle and collision avoidance; (g) integrated motion control; (h) spark-ignition engine control and (i) transmission clutch torque control.

Even with the present focus on automated driving, it should be clear that all are relevant to some extent. Of least immediate relevance are suspension control (a), powertrain control (h), (i) and perhaps (b) - worst case manoeuvring aimed at finding worst cases for vehicle systems to be tested under; for automated driving such cases are the more complex driving scenarios, for which a simple "worst case" analysis may not be credible. Prior research into minimum time manoeuvring (lap time optimization for race cars) is perhaps relevant, as it concerns "optimal driving" under friction limited conditions, so the research methods may be applicable in part in areas such as collision avoidance. Without going deeper into the specific areas, the conclusion is that most of 'basic' control 
methods of vehicle system dynamics are relevant to the automation of driving systems - we should beware of creating artificial separation between 'modern' and 'traditional' problems of automation and control.

We should also be wary of somewhat artificial boundaries between tactical and control layers: improving the precision of even basic manual control functions can have an effect on the way drivers interact with vehicles. A relevant example here is the Electrical Power Assisted Steering (EPAS), which has been available in cars for some time. This has recently emerged as Electro-Hydraulic Power Assisted Steering(EHPAS) for heavy trucks [35]. EPAS and EHPAS can not only improve the precision of vehicle control and the comfort for the driver, but also meet the higher requirements put on the steering system from Partial Automation and Conditional Automation. This comes from the substantial improvements in force feedback provided to the driver ([36], [37]). Basically, with the same philosophy, the improved precision of braking (electro-hydraulic, electro-mechanical, full electric control of torque), traction and torque vectoring systems [38] can also contribute in enabling control level functions to migrate vehicle capabilities up to the tactical level. Or, in other terms:, without adequate precision at the control level, such migration towards 'dynamic intelligence' on the vehicle may be difficult or impossible to achieve.

There is another, perhaps unexpected, link between control-level technology and high level automation. For High and Full Automation the need for mechanical connections between driver and actuators (e.g. steering) becomes less relevant; in Level 4 (high) automation, there is no expectation for the driver to take over control in an emergency, so why impose the constraint of retaining mechanical connections? This immediately opens up the scope for a full by-wire automated car control similar to the by-wire approach of modern aircraft. As for an aircraft, the by-wire technology is designed to handle the major control functions using electronics only. The research and development challenge is to make use of system-level redundancy to prevent serious control degradation in the event of any single point failure. Certainly there are considerable safety implications associated with by-wire controls [39], but these are somewhat dwarfed by the other safety and reliability challenges introduced by Level 4 driving automation.

\subsection{Highly Automated Driving}

As mentioned, currently there are several commercial and academic groups engaged in developing highly automated research and development platforms, some expected to achieve Level 4 capability. There have also been a number of larger-scale demonstration systems and there are even some niche commercial applications already in service. All of the systems which expand beyond a single vehicle concept make an attempt to reduce the demands of tactical driving, and in many cases do this by simplifying the driving environment and hence reducing the demands on the intelligent vehicle. In August 1997, a large research and development project ended with a live proof-of-concept demonstration of a prototype automated highway system, with 20 self-driving cars operating over a four-day trial period on dedicated lanes of Interstate 15 in San Diego, California [40],[1]. With speed and directional control both automated, the system used permanent magnets embedded in the road acting as a virtual guide-rail. Of course, manually driven vehicles were excluded from these dedicated lanes, and combined with the magnets we immediately see that the everyday demands of tactical driving are mostly removed.

Because platoons of vehicles are to be moving together, there is the possibility of an instability occurring; when a lead vehicle accelerates for example, the following vehicles may need to accelerate progressively harder to catch up, leading to a slinky effect or string instability. This can be unnerving, and could lead to collisions whenever the lead 
vehicle brakes suddenly. Interestingly, the 1997 demonstration showed that automated vehicles can control platooning more precisely than human drivers. With close spacing achieved at highway speeds, the demonstration showed potential advantages for increased traffic flow via reduced aerodynamic drag among the following vehicles.

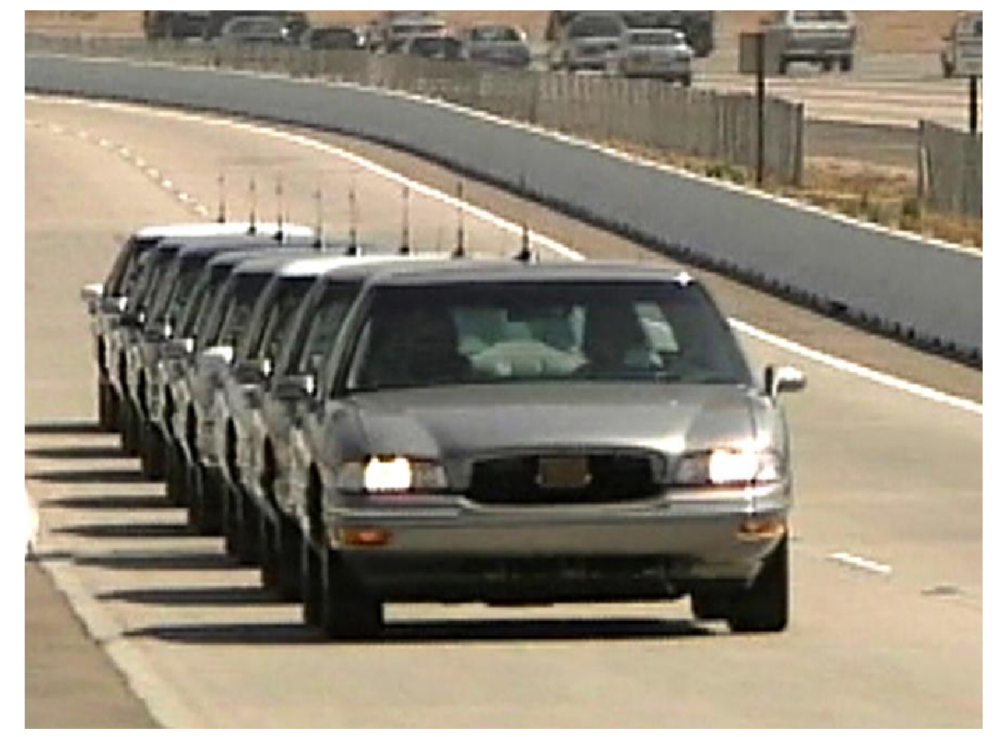

Figure 6. Live proof-of-concept demonstration of a prototype automated highway system, with self-driving cars operating over a four-day trial period on dedicated lanes of Interstate 15 in San Diego, California 1997 (Photo: California PATH Program).

Again, in a recent EU project, Safe Road Trains for the Environment (SARTRE) [41], led by Ricardo UK, a platoon approach was used. In this system, a heavy truck with a human driver at the wheel acts as platoon leader was followed by suitably equipped and highly automated passenger vehicles. With highway speeds and close spacing, this mother-duck/baby ducks scenario allowed drivers of following vehicles to enjoy eyes-offthe-road time. The approach is broadly feasible on existing highways as it simplifies the driving environment for each baby duck, mainly at the level of tactical driving. SARTRE is an interesting example of using a novel approach to deal with the complexity of the normal traffic environment.

A third and very different example of controlling the driving environment goes beyond the demonstrations of concept and further highlights the role of ensuring safety via a highly controlled infrastructure. Personal Rapid Transit (PRT), or podcar, is a public transport system featuring small automated vehicles operating on a network of specially built guide ways. Since 2011, PRT pods have ferried passengers between Heathrow Airports Terminal 5 and a nearby parking area [42]. With speeds up to $25 \mathrm{mph}$, self-driving electric cars run on dedicated tracks, with boundary fences, concrete barriers that separate adjacent lanes, CCTV and other real-time monitoring, plus the safety net of an independent collision avoidance system within the pods. The system, akin to a flexible rail system, is a long way from the freedom of general purpose self-driving cars, but it demonstrates real value to customers and is already economically viable. PRT systems require dedicated infrastructure which is both costly and challenging [43]. A different pod system is planned to ferry passengers between the rail station in Milton Keynes and nearby shopping centres [44]. Fully autonomous pods will be trialled in Milton Keynes in 2015 by the UK Transport Systems Catapult. Ultimately ferrying passengers between 


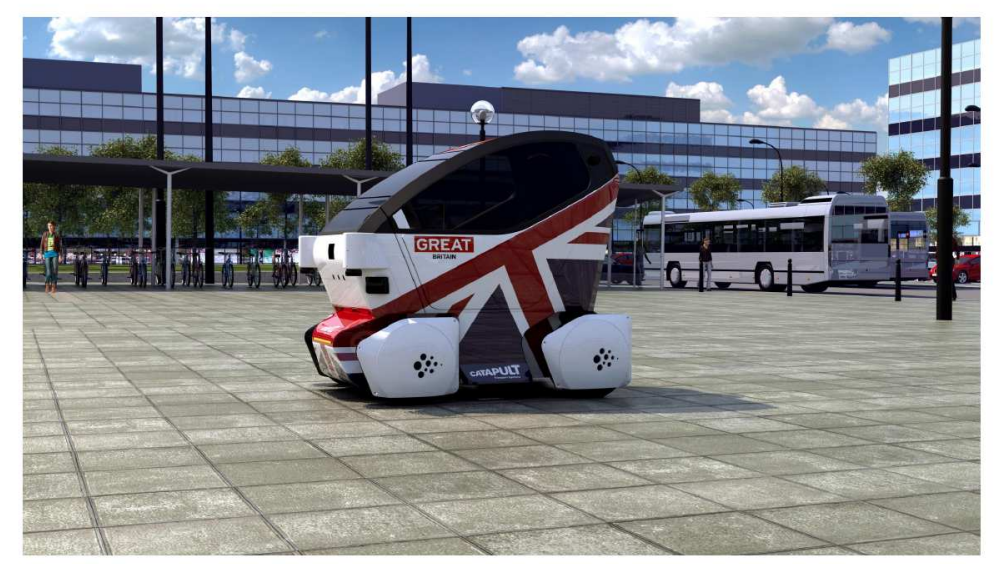

the railway station and nearby shopping centres, the pods will run at up to $24 \mathrm{kph}$ and be tested on a pavement route agreed with Milton Keynes Council to see how they interact with the public.

Figure 8. Milton Keynes Pods on pavement (Picture: The Transport Systems Catapult UK).

Whether in platoons, niche commercial rail-like systems, or at the level of an individual highly-automated vehicle, there is a need for a consistent control architecture. A simplified architecture for a 'typical' autonomous vehicle guidance system is shown in Figure 9. The guidance system is mainly composed of four modules: a trajectory/mode generator, a trajectory/mode replanning, a low-level control system, and finally the vehicle and the real-time environmental model. In this approach an external control reference is used 
alongside a path-following algorithm, splitting the motion control into two parts. This is a common approach but it is not without its possible drawbacks - see in Section 8. In any case, the trajectory generator and trajectory/mode replanning roughly equates to the tactical driving elements of Figure 2, while the low-level control block is obviously equivalent to the control level of Figure 2. For integrated systems of the type mentioned above there are additional layers required for the coordination and integration. And, as with the rail-like systems mentioned above, there is a further need for one or more layers of collision protection. Of course, the systems for both automatic driving and collision protection have an absolute need to directly sense and interpret the environment, and the collision protection system requires a threat evaluation function. We now consider environmental sensors and their use.

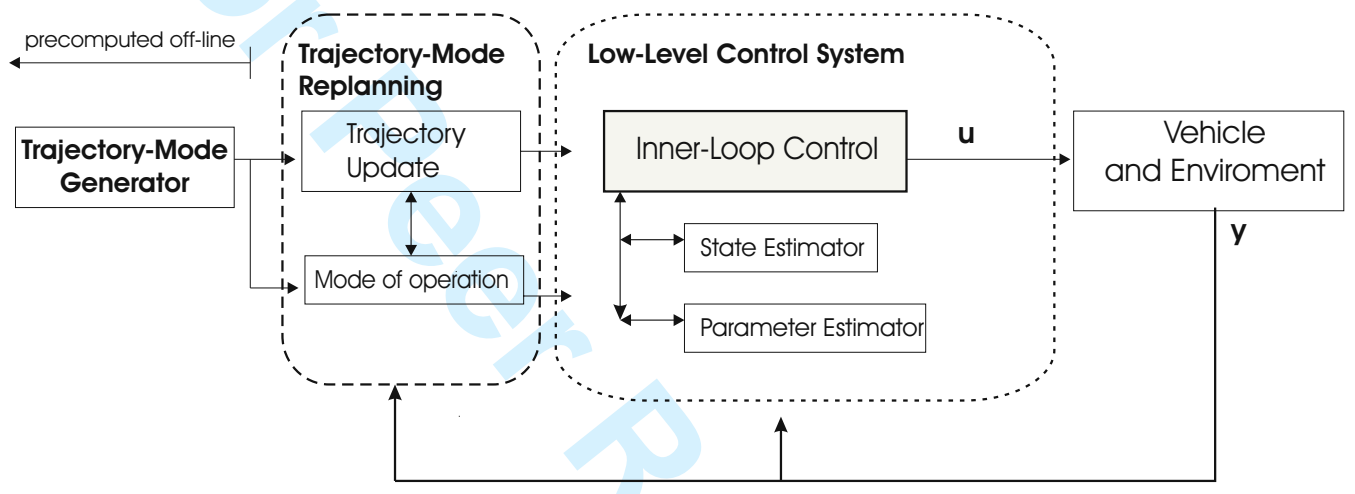

Figure 9. Simplified architecture for fully autonomous vehicle guidance system. (from Falcone [45]).

\section{Environment Sensing and Motion Tracking}

At the most basic level an intelligent vehicle with automated driving capabilities needs to know where it is in the world, using some local coordinate system. It also needs to know the geometry of stationary features such as road boundaries and intersections, i.e. a local map. The map may be ad-hoc - created in real-time from sensors - as for example when an LKA system uses a suitably calibrated forward pointing camera to determine the locations of lane boundaries relative to the vehicle. Or a stored map may be used. In this case the precision of both the map and the vehicle location relative to the map are critical for motion planning and control. Currently there is a lack of publicly available maps of sufficient quality for direct use by automated driving systems, perhaps since the requirement for intelligent vehicle control is relatively new. This fact may have an important influence on the development of automated driving systems.

\subsection{Mapping and Localisation}

\subsubsection{Ad-hoc Mapping}

As a simple example, consider the position and mapping requirements of ACC. In the 'world of ACC', the ego vehicle drives in a single lane with limited curvature; the relevant 
elements of its environment are the lane markings and the vehicle in front (which may or may not be within range of the forward radar). Ego vehicle speed is estimated from the driveline, range and range-rate are deduced from a forward pointing radar, and road geometry is used to determine if a particular radar reflection comes from a vehicle in the same lane [46]; machine vision may also be used as part of this confirmation process. In this way a local map is created in an ad-hoc manner, and in particular it is not stored for later use. Although ACC (and LKA likewise) provide only a low level of driving automation, they share a number of the mapping and positioning requirements of other more highly automated systems. In particular, the control algorithms are based on a limited concept of the surroundings, in this case the world to the left or right of the current driving lane is conceptually outside the domain of ACC control and vehicles that 'cut in' from the left or right are recognized as new objects that appear into the ACC world. Of course the capability to pre-empt and accommodate cut-ins exists as a functional extension for ACC (hence expanding the relevant environment of the system).

Another example of ad-hoc local mapping comes from the MIT team that competed in the DARPA Urban Challenge [47]. As shown in Figure 10, a large number of sensors were used, in addition to the given 'route network definition file' (RNDF) and mission data file' (MDF) used in route planning. These comprise odometry, inertial, GPS, lidar, radar and vision. Distance measurement from the vehicle (onboard odometry) can be from wheel or transmission speed; however this accumulates errors under even mild acceleration or braking and must be carefully calibrated for rolling radius, taking account of any changes in inflation pressure and vehicle loading. Two different GPS systems were used (one with an integrated inertial navigation system, INS), and three different ranging or imaging systems were also employed for tracking lanes and other vehicles as well as detecting drivable surfaces and obstacles. When the local map is connected to the world data from the RNDF file the result is an enhanced map, though again it appears that the map is not stored for subsequent missions; for the 'real world' of public highways it clearly makes sense to store and possibly share these kinds of data for reuse on future trips. It is worth noting that GPS was used as a reference to build local maps, but not directly for guidance, due to accuracy and availability concerns - see below.

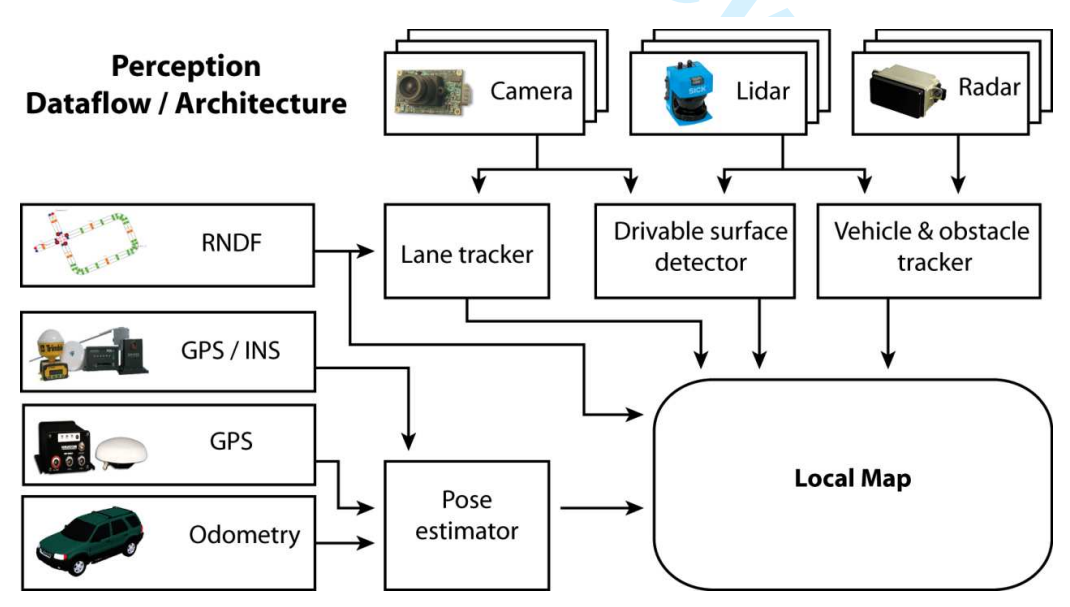

Figure 10. Hierarchical structure of the driving task including attention switching (from Leonard et.al. [47]).

From work by Paul Newman and his group in Oxford, it has been shown possible to use computer vision for building a map without any prior information [48]. Although 
this may have limited direct application to enhanced mapping of highways (accuracy levels are quoted as several metres extracted from a round trip of several kilometers) the power of the method is indeed worth considering for specialist mapping applications. The system uses stereo vision only and manages to 'glue together' successive locations of the vehicle into a comprehensive map. The system relies on static objects in the world being recognised in successive images, so that the change of pose (position and orientation) of the vehicle is found from the vision system and hence integrated into a time history of successive poses. And since the objects used for connecting the poses are an inherent part of the process, they can be fixed in the world. The system reduced drift by having the vehicle return to the start location and use loop closure as a constraint. It might be that this type of approach would be more accurate if it used a limited number of surveyed waypoints that could be recognized easily by the vision system. This would then represent a special type of map enhancement with the waypoints existing on a pre-defined low-resolution (but high accuracy) map.

Satellite positioning (GPS, or more generically and correctly Global Navigation Satellite Systems, GNSS) is ubiquitous but not always adequate for ad-hoc mapping nor for vehicle guidance. As mentioned, the MIT team from the DARPA Urban Challenge [47] did not rely on GPS for guidance, due to its relatively low accuracy and poor reliability of service, especially in an urban environment, where signals are blocked or suffer path reflections. While GPS is normally adequate for navigation systems, common experience shows that errors can easily exceed $100 \mathrm{~m}$. There are ways in which this baseline accuracy of GPS can be improved; for example Satellite-Based Augmentation Systems; Geostationary Navigation Overlay Service (EGNOS) or the Wide Area Augmentation System (WAAS) may be used to improve both the accuracy and integrity monitoring of GPS. However, even with such enhancement, absolute positioning accuracy is $\lesssim 3 \mathrm{~m}$ Assuming a sufficient number of satellites are visible, and in the absence of excessive multi-path errors (reflections from buildings etc.) RTK (real-time kinematic) differential corrections can be made to reduce errors to a few centimetres. But these systems are expensive and, while routinely used in professional mapping and surveying, they are not viable for commercial use on intelligent vehicles.

\subsubsection{Enhanced Digital Maps}

Humans don't need to learn the local geography in order to drive, and people can successfully drive in unfamiliar environments; but for real-world driving automation there are overwhelming advantages in using detailed stored digital maps to supplement what is available from published sources. Eventually such enhanced digital maps may be published, though a great deal of effort is required to establish this across the majority of the road network. Clearly it is wasteful and inefficient to build a new map every time the vehicle passes through a road segment, unless perhaps the level of detail is very low. Indeed, for the DARPA Grand Challenge, a critical success factor was the extent to which teams created enhanced maps prior to the actual drive [7], [49].

The requirement for map accuracy derives from the application, and in the case of automated driving functions the 'standard' requirement is lane-level accuracy. This is needed to support any plausible tactical capability, to determine which lane the ego vehicle is in (absolute error $\lesssim 1 \mathrm{~m}$ ) and hence plan for lane changes or take account of lane-specific traffic rules (e.g. right-turn only). This level of accuracy is also sufficient for other aspects of tactical driving, e.g. to plan deceleration on the approach to an intersection. Of course $1 \mathrm{~m}$ accuracy is not sufficient for the control level of driving; for example machine vision is still required to support lane-keeping systems, giving positional 
accuracy in the lane within a few centimeters.

Current publicly available road maps do not have this required accuracy; standard digital highway maps have an accuracy of only around 5 to 20 metres [50]. Furthermore they do not record the level of detail required - even to specify the number of lanes and whether the road has a central median is beyond the scope of existing digital maps. Hence some form of enhancement is needed via additional data gathering.

It is possible to automatically enhance existing low-resolution maps using data from dedicated test vehicles. An example is described in [51] using a survey vehicle and making use of publicly available maps from OpenStreetMap (OSM) [52]. At the core of this work is a commercial 'survey-quality' GPS system (Applanix POS-LV 220 inertial GPS navigation system) used to define the pose of the survey vehicle. The system incorporates two GPS receivers, inertial guidance and a rotary encoder for one of the non-driven road wheels. With additional sensors to capture road markings (camera and LIDAR) is was shown possible to produce map data with an error range $\pm 0.22 \mathrm{~m}$ and with mean absolute errors in the order of a few centimeters. This compares with a more standard survey system in [53] using the same type of GPS navigation; here - without any prior mapping - the stereo-vision system is capable of discerning features to within around 0.3 $\mathrm{m}$ (RMS) horizontally and $0.5 \mathrm{~m}$ vertically. Thus lane-level accuracy is available using only survey-grade GPS and optical/LIDAR imaging to create the required maps, without needing any fixed base station, and this remains true even when GPS coverage is limited [53].

A basic but important requirement for future use of stored enhanced maps is that they are maintained (reflecting updates to roads and lane layouts) and made available in a seamless way. Even outside of transportation applications, new mapping, data management and search techniques - in the form of Geographical Information Systems (GIS) has started to revolutionise the way maps are assembled [54].

Thus current maps are woefully inadequate for driving automation. Existing GIS data sets represent roads as a coarse series of nodes and shape points, which are connected by segments, and with no other salient features. In the future it should be expected that a great deal more data can be recorded and verified in available GIS data sets, including lane number, land widths, median type and dimension, type and size of shoulder, quality of lane marking, presence and type of crash barrier, presence of rumble strip etc., all of which are relevant to driving automation.

\subsubsection{Localisation}

The position and orientation (pose) of an intelligent vehicle is needed in real-time (i.e. with limited and preferably constant time delay in processing). While the 'obvious' way to determine the position of an intelligent vehicle is via satellite navigation systems, we saw above that accuracy and availability are insufficient. If an accurate map exists, GPS corrections may be applied based on the assumption that the vehicle is on the road (map matching) though best-case positioning accuracy is no better than around $5 \mathrm{~m}$. Further improvement can obtained from onboard odometry (distance measurements based on speed over ground or rotation of the road wheels) but lateral positioning relative to the road centre is not greatly improved. Another opportunity for real-time enhancement of positioning accuracy is to register fixed beacons or markers in the environment. These might be permanent magnets, visual markers readable by cameras, or they could be RFID (radio frequency identification) transponders. In [55] it is shown to be potentially feasible to achieve lane-level positioning using passive RFID tags in the road combined with electronic readers attached to the enabled vehicles. Position errors less than $1 \mathrm{~m}$ 
are predicted, though the study is limited to simulation.

It was mentioned previously [48] that stereo vision may be used to identify relative positions of fixed objects from frame to frame in an onboard visions system, hence identifying change of pose; but when there is considerable initial uncertainty in the reference object's location there is no guarantee that vehicle pose can be reliably estimated. It may be that a combination of lane markers, machine readable reference points, plus enhanced maps could provide the necessary reference for a vision system, but it seems clear that further research is needed on whether machine vision is sufficiently robust for the necessary localisation problem.

Another approach is to use DSRC (Dedicated Short-Range Communication, a secure form of wifi for highway applications) to enhance positioning. In [56] the DSRC service is used to broadcast a differential correction. In their experiments the additional service did indeed provide adequate enhancement but the reliance on GPS service remains troublesome. In [57] a rather novel approach is considered without GPS (other than to provide ground truth for evaluation). The system uses two fixed roadside DSRC base-stations within range of a DSRC-equipped vehicle (Figure 11). The base-stations broadcast their respective positions and the lane geometry as digital data. The wireless carrier frequencies differ based on doppler shift and vehicle location relative to the beacons. Using vehicle odometry to determine speed, the location of the vehicle can be determined. Success seems to depend on the distance between the base stations and their location relative to the vehicle, but the authors report accuracy that increases with vehicle speed and typically with RMS errors less than $0.5 \mathrm{~m}$ above very low speeds.

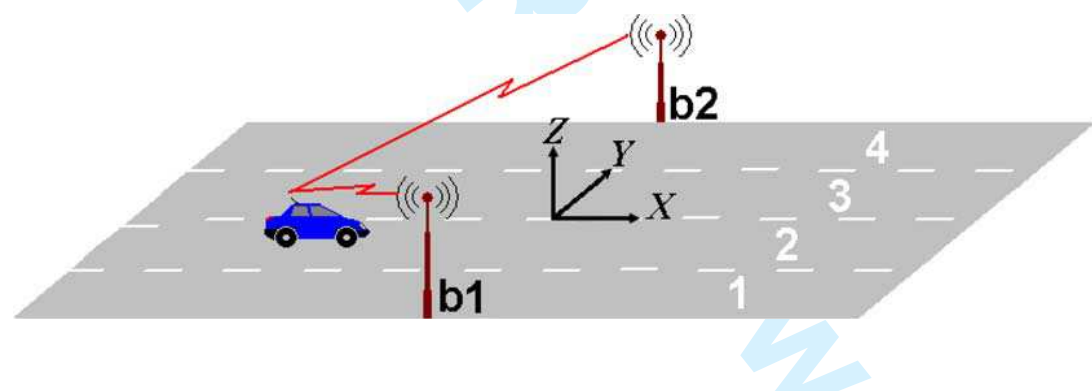

Figure 11. Lane-level localisation using DSRC beacons (from Alam et. al [57]).

Much greater precision is possible using regularly spaced permanent magnets fixed in the centreline of the road [58]. In the work of the California PATH Program at the University of California vehicles sensed lateral position relative to these closely spaced magnetic markers, for which lateral offset directly fed into the steering control algorithm. Experiments showed that lateral tracking errors remained less than $10 \mathrm{~cm}$ under most conditions, and generally less than $15 \mathrm{~cm}$. Compared to other current approaches summarised above there are both advantages (high precision and availability of signal) and disadvantages (inflexible, does not support continuous localisation away from the chosen lane). Perhaps the clearest point of difference is that these markers were used directly for lateral control, rather than for lane-level positioning to support tactical aspects of driving automation. Current approaches rely much more heavily on GPS (when available) and vision systems to enhance the maps and also provide control level feedback (as in lane tracking).

It is worth mentioning that, just as GPS availability is a serious concern, so is the availability of other positioning systems. For a 'fleet' of embedded magnets, these would 
need to be maintained in a routine way if used on the highway; similarly, existing lane markings need to be repaired or replaced to support tracking by machine vision cameras. This is not always the case - for example, on certain road types and in adverse weather conditions, availability may be as low as $20 \%$ [32] though for highways it is closer to 90 $\%$, see Figure 13. Clearly, wet roads at night are a particular problem for machine vision systems.

\begin{tabular}{|l|l|r|r|r|r|}
\hline \multicolumn{2}{|c|}{} & \multicolumn{2}{l|}{ day } & \multicolumn{2}{l|}{ night } \\
\cline { 3 - 7 } \multicolumn{2}{|l|}{} & \multicolumn{1}{l}{ clear } & wet & \multicolumn{1}{l|}{ clear } & \multicolumn{1}{l|}{ wet } \\
\hline Type A & rural & 90 & 90 & 90 & 20 \\
\hline & urban & 90 & 90 & 90 & 20 \\
\hline Type B & rural & 85 & 85 & 90 & na \\
\hline & urban & 70 & 70 & 65 & na \\
\hline Type C & rural & 50 & 50 & 50 & 20 \\
\hline & urban & 40 & 40 & 40 & 20 \\
\hline
\end{tabular}

Figure 12. Percentage availability of lane departure warning camera system used in South-East Michigan; Type A: Multi-lane divided, Type B: Multi-lane undivided, Type C: Two-lane undivided] (from Gordon et. al [32]).

Overall the tasks of mapping, map enhancement and localization of the ego vehicle relative to a local coordinate system remains a challenging area. Accurate information of this type is critical to all forms of automated driving control, though of course it is not sufficient. As noted, humans are capable of driving in the absence of such maps, particularly helped by familiarity with common patterns of road design. Of course accurate mapping and localisation is only sufficient for automated control if there are no other objects sharing the road surface along the planned path.

\subsection{Object Detection and Threat Assessment}

Sensor types for object detection and collision avoidance overlap with those used for guidance in driving automation. Additional types include short-range proximity radar and ultrasonic sensors, also for short range proximity detection. Unlike vision, LIDAR and scanning ACC radar, these proximity sensors have no way to recognize or classify objects, other than by context (e.g. because driver has switched on a parking aid, reflections indicate barriers or other vehicles etc.).

Autonomous systems for collision protection are being developed irrespective of any wider agenda to develop highly automated vehicles. An example is the Volvo City Safety [59]; the system monitors threat vehicles ahead via a laser sensor and brakes without driver intervention when an imminent crash situation is detected. This particular system operates specifically at short range and low speeds, and is from the general class of Automatic Emergency Braking (AEB) systems - Section 2.1.

There are clear overlapping demands between vehicle guidance and obstacle detection in the longitudinal direction. For normal driving, ACC radar or LIDAR detect vehicles in front, with the same or similar requirement as for AEB [22]. In both cases there is a need for a threat assessment (and intervention) algorithm, based on speeds and range, but the details will be different, particularly for the threshold levels to trigger a response. Thresholds for AEB activation are based on metrics similar to Time To Collision (TTC) or Last Time To Brake (LTTB) [60]. TTC uses range $R$ and range-rate $\dot{R}$, and is defined 


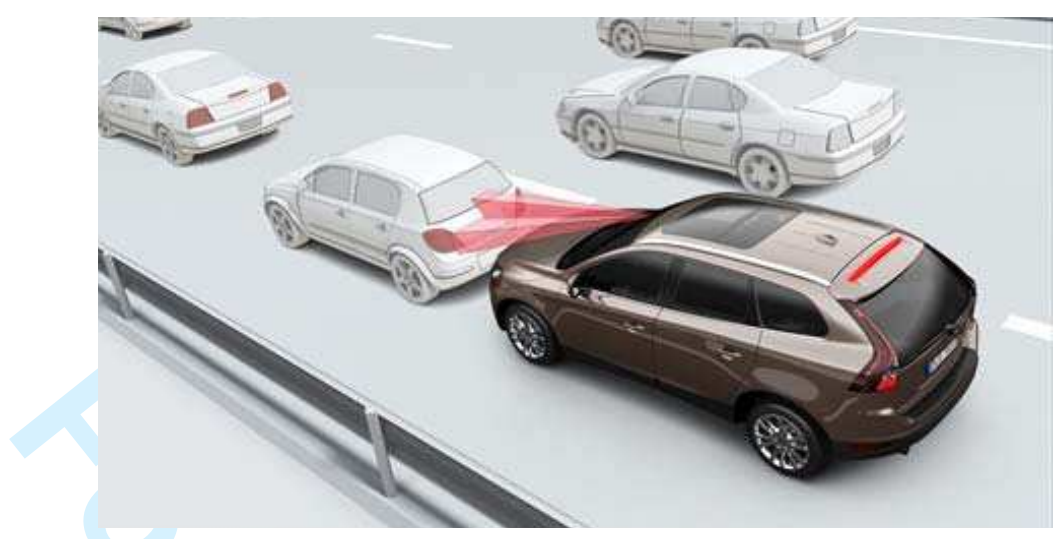

Figure 13. Automatic braking to avoid low-speed frontal crashes (Volvo City Safety system - from Distner et. al [59]).

as the time to collision assuming constant speed of both vehicles, i.e. $T T C=R / \dot{R}$. LTTB uses more detailed information, including estimated surface friction, but in either case there will be uncertainty over any deceleration of the lead vehicle and AEB may not be expected to always prevent collision; but in all cases the relative speed at impact will be reduced by the intervention.

More capable collision protection systems require more sophisticated sensing and sensor interpretation, including multi-sensor fusion algorithms. For example the Volvo "Collision Warning with Full Auto Brake and Pedestrian Detection" (CWAB-PD) uses radar for increased range and monovision cameras for validating the type of object [61], [60] and hence can deal with a wider range of forward collision situations.

Threat assessment from the lateral direction is more specific to collision protection. A number of 'blind spot warning' systems have been on the market for some time; when there is another vehicle nearby in the adjacent lane a warning light illuminates to alert the driver that a lane change would not be safe. Alternatively a haptic signal may be used via the steering wheel [62]. These systems are activate by radar or cameras, which may also be used to trigger active steering resistance or path correction in case of an unsafe lane departure.

There are various other collision prevention systems for specific crash types, for example warning and intervention to avoid hitting a pedestrian when reversing a vehicle. In [63] a number of these systems, all using ultrasonic sensors, were evaluated. In some cases their effectiveness questioned, mainly due to short range limitations, and especially those using sensors developed as parking aid systems; these detection systems sacrificed detection distance and height in order to suppress false or nuisance alarms. Again, while guidance and protection involve similar functionalities, their precise requirements are different and the systems should normally be developed independently.

Collision protection is often based on assumptions of deterministic intent of the threat object (e.g. constant speed or deceleration of a lead vehicle). However this is not necessarily reasonable or useful in the area of preventing car-to-vulnerable road user (VRU) accidents. In [64] there is an attempt to characterise, in a non-deterministic model, the likelihood of a pedestrian entering the road from based on various factors: car speed and its distance to the point of potential collision, pedestrian distance to the road, plus pedestrian speed and head orientation. Such a predictive algorithm could be worthwhile in reducing crash risk by alerting the driver or any AEB system. On the other hand it is not likely that a Level 4 automated vehicle would entirely rely on such a system, and yet 
a deterministic model would clearly be inadequate. This and similar crash modes may in the future define the environmental limits on the safe use of highly automated vehicles.

\section{Safety and the Role of the Driver}

Safety is perhaps the biggest single concern when considering the automation of driving. Each level of Figure 5 brings its own safety concerns, and in particular the question of 'what happens if something goes wrong'. It is hard to believe that a mother would send her child to school in a Level 5 self-driving car without severe trepidation, unless there were a trusted human being as part of a 'backup plan'. And the driver of a Level 4 platoon vehicle would surely be sensitive to whether the lead vehicle has a human driver (as in SARTRE) or not. It appears that the topic of risk and safety of highly automated vehicles is wide-open, given that predictive safety evaluation of road vehicles is at a very early stage, and there is little real-world experience to draw on.

At Level 0 there is of course a lot of experience, and it is easy to underestimate the safety performance of manually driven vehicles. According to US traffic statistics in 2012 [65], out of roughly three trillion vehicle miles travelled, there were a total of 5.6 million police-reported traffic accidents, 30,800 of them involving a fatality. This equates to roughly one crash per 500,000 miles and one fatal crash per 100 million miles. This compares to the currently quoted million-plus miles of accident-free (supervised) travel for Googles self-driving cars, where the development team selects roads and timing, intervenes when necessary, and presumably does so with high attention levels. For humans, tactical driving requires experience and attention. It is also the level that is most demanding for highly automated cars. It seems infeasible to replicate all aspects of human driving at the tactical level, at least not without artificially intelligent computers that are beyond anything currently proven. We might formulate a Turing driving test on this topic: if a self-driving car behaves in all traffic conditions such that other road users cannot tell any difference from a human driver, then we might assume that we have a safe and acceptable system. Of course the traffic conditions should include complex and dynamic scenarios.

Example complex scenarios are considered in [66] - see Figure 14. That work relates to highly-capable driver assistance systems: the driver support function (so-called 'codriver' in this case) needs to anticipate the intended manoeuvre and cannot do so unless the driver's goal is known (in this case either $\mathrm{a}-\mathrm{b}, \mathrm{a}-\mathrm{c}$, or $\mathrm{d}$ ). Each goal/manoeuvre has risks associated with it, and the co-driver must correctly anticipate in order to monitor traffic and reduce risk. This is an immensely difficult problem for which the support system could potentially increase risk due to unpredictable human-machine interactions.

This example illustrates that risk and safety prediction is highly challenging and not just for highly automated vehicles. The HAVEit project [67] also considers the concept of driver support from a co-driver, including aspects of adaptation to risk and flexible levels of automation (see also Figure 3). But again the work does not provide predictive measures of crash risk.

For active safety systems there have been a number of studies assessing the effects on crash risk. When modalities are relatively simple it is possible to assess crash benefits by fusing a wide range of data sources, combining these via Monte-Carlo simulation - e.g. for Lane Departure Warning systems [32]. Real-world statistical analysis can be used for systems that operate very close to potential crashes. For AEB systems, it was reported in [68] that there is a significant reduction in crash numbers for the relevant 'striking rear end' crash type; in $50 \mathrm{~km} / \mathrm{h}$ speed areas the reduction was $54-57 \%$, while over all 


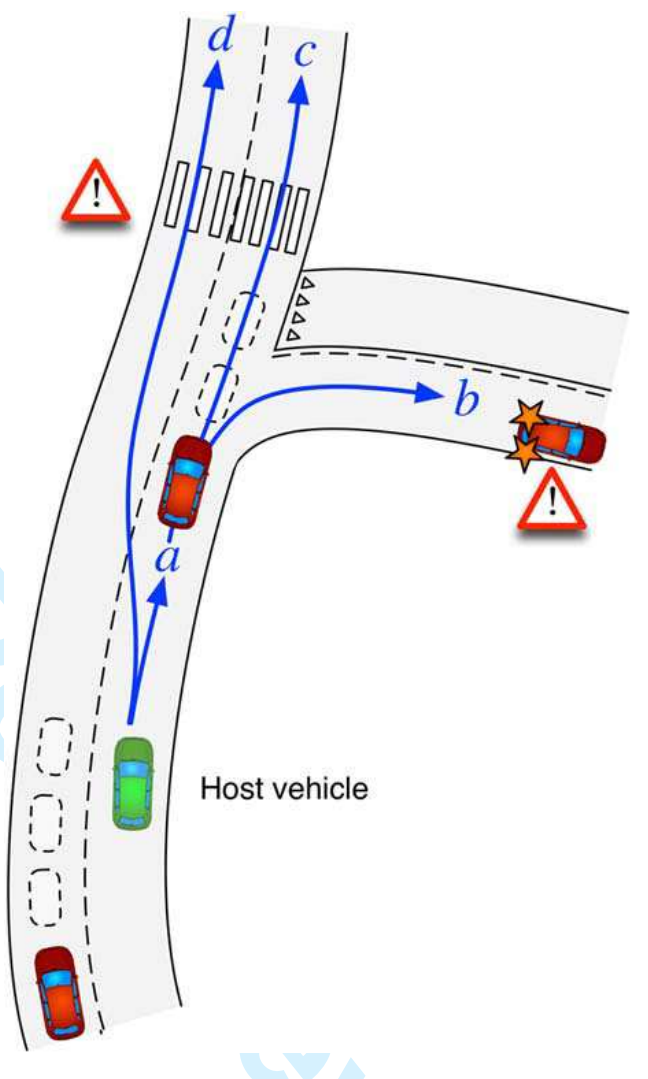

Figure 14. Complex highway scenario with multiple feasible outcomes (from Da et. al [66]).

speed limits the reduction was $35-41 \%$. The work was based on Swedish police-reported injury crashes between 2010 and 2014. A similar trend was reported in [69] for the Volvo City Safety system: the number of claims for property damage liability were $16 \%$ less for (equipped) S60s than for other (non-equipped) mid-size luxury cars.

Given that we cannot yet put human-like driving intelligence into automated driving systems, there are two complementary ways forward towards achieving high levels of automation. First, we can make best use of the available safety net of collision prevention systems, such as AEB. Over time, using crash data and improved scientific analysis, we can further evaluate and verify the safety benefits of such systems. These 'safety nets', developed to protect from errors by human drivers, can also provide protection during various levels of automated driving; thus driving safety benefits can be extended and validated in real-world studies. Secondly, we can simplify and regulate the driving environment to the point where crash risk can be evaluated in an objective way ahead of deployment.

\section{Optimal Driving Control}

Human driving may be far from optimal [70] - for example control time-delays are long compared with automated systems, and drivers' attention may be intermittently 
switched away from the driving task. Steering control typically has low bandwidth and maintaining lateral lane position is not necessarily a challenging control task, whether for a human or a machine. Under normal conditions, human driving is effective, and one might question whether automated driving systems should aim for optimality in any particular sense, even though a number of studies into synthetic driving systems (driver models) use optimal control methods at their core [34]. One might go further and seek to design automated speed and steering control systems that perform in naturalistic ways - sufficiently akin to human driving so as to be acceptable to vehicle occupants and to other road users. This is contrary to the idea of imposing a rigorous control regime positioning the vehicle as precisely in the lane centre as possible and maintaining precise headway - where such behavior may appear alien and distracting to other road users.

This concern suggests that research may be worthwhile into understanding how automated driving control should be capable of mimicking human behavior, at least in certain driving scenarios. But research is also needed to explore the benefits of precision and optimality when automating different aspects of the driving process - to improve safety during normal driving, to provide effective protection in safety-critical situations, and more generally to improve "softer" aspects of vehicle system dynamics, such as comfort and fuel economy, as well as reduced carbon emissions. Travel-time efficiency is also worth considering in this context.

The very concept of highly automated (self-driving) vehicles implies time saving benefits to drivers - being able to focus on other activities while automated systems perform all tactical driving and control functions on the vehicle. This is true, whether or not the stated aim is for safety improvement, fuel saving etc. Further, during normal driving scenarios, these systems have the potential to fuse real-time environmental information (e.g. traffic and topography) with data about the vehicle status (e.g. state of charge of the battery) to implement efficient control strategies that are beyond the interest and capability of human drivers.

One example of automated driving optimisation that requires such data fusion is the "pulse and glide" strategy for longitudinal control, aimed at minimising fuel consumption [71]. This expands on the simple idea that running an internal combustion engine close to its most efficient operating point will improve fuel economy. In the absence of a continuously variable transmission, a way to achieve this is to run the engine intermittently at a higher power in order to reach the efficient operating point. Even allowing for transient reductions in fuel economy, the pulse and glide strategy may be capable of producing around $20 \%$ improvement in fuel economy. But this is only possible if the intermittent acceleration and deceleration does not disturb traffic flow or cause other drivers to react. While the control concept may well suffer from obvious problems, particularly the discomfort of passengers [71], the fact that local vehicle optimal control can benefit from being sensitive to the interaction with the vehicle environment is interesting and probably quite general. The example can be described as having a 'weak' connection to the vehicle environment. Similar weak connections exist around traffic signal control and cooperative ACC (CACC - see below) which aim to smooth traffic flow, increase highway capacity and reduce transportation energy consumption with particular emphasis on vehicle communications technologies, either between vehicles $-\mathrm{V} 2 \mathrm{~V}$, or between vehicles and infrastructure - V2I [72].

In other concepts, the traffic and physical infrastructure may require a much stronger connection. 


\subsection{The Automated Highway}

Optimization of strategic driving in response to prevailing traffic conditions (real-time route planning) is a well-known problem in traffic network analysis [73] and since this problem is very much aimed at the upper level in Figure 2, there is little relevance to the optimal automation of driving processes, except for any resulting interactions between network level "control" and individual vehicle control. Referring again to Figure 2, until now we have considered the functions to be localized within the host vehicle, even though contributions from both human driver automated driving systems may be involved. From the alternative perspective of "Automated Highway Systems" (AHS) or "Intelligent Transportation Systems" (ITS), there may be scope for the highway system to take over both tactical and strategic functions. Of course we always expect the low level control of vehicle systems to be handled locally.

Within infrastructure-centric control, common applications relate to traffic flow improvement via static infrastructure components, especially traffic signals [74], [75], [76]. Recent interest in highly automated vehicles has led naturally to traffic flow optimization studies that assume direct control of automated vehicles, and this requires a switch towards granting responsibility for tactical driving to a centralised transportation control system.

A conceptual example of such centralized control is the Integrated Roadway/Adaptive Cruise Control System (IRAC) [77] which assumes infrastructure-based controls for speed distributions along highway lanes as well as ramp-metering, i.e. traffic signal control of vehicles entering the highway. This study was based on traffic simulation, assuming the use of DSRC communication, and predicts improvements to traffic flow. More extreme example of centralized traffic control are presented in [78] where the authors propose a coordinated traffic control scenarios, where lane-changes are executed in the path of cooperating oncoming vehicles. Such scenarios are somewhat futuristic in terms of the level and scope of control that is required and raise questions over the effect of any singlepoint of failure - when one vehicle fails to cooperate there is near-certain collision. The optimization problem is limited to a local group of vehicles (around 40) and is applied at the level of discrete decision making. While the full idea is somewhat implausible (e.g. the number of collisions predicted are not vanishingly small) they do raise the potential for a new approach where a "smart infrastructure" provides an additional layer of tactical support, reducing the tactical driving demands on the individual vehicles.

\subsection{Platoon Control}

Travel efficiency was also the primary motivation behind the use of precisely controlled platoons as developed and demonstrated by the California PATH program described in Section 3.2 [40], [1]. Platoons are clusters of vehicles that synchronise their motion to form a closely-moving pack, acting as a single multi-vehicle 'object' on the highway. In the PATH project the assumption was that such a platoon will move on a dedicated lane within a tightly controlled AHS environment.

Several concepts from platoon dynamics, particularly the need to use wireless communication to suppress longitudinal disturbances (string instability), have led to research and development in the area of Cooperative ACC (CACC) where it is assumed that lateral control comes from the human driver but a self-organizing platoon emerges from local headway control (as for ACC) with the addition of cooperative elements which are mediated by wireless communication. One may think of CACC as a type of 'optimised' ACC system, even though optimal control methods are not necessarily used in the controller 


\section{Vehicle System Dynamics}

design. Experiments with this type of system took place during the 'Grand Cooperative Driving Challenge' (GCDC) where several vehicles were expected to cooperate in order to perform cooperative adaptive cruise control (CACC), [79]. While a number of practical and technical problems were revealed in this work, the basic feasibility of CACC is not in doubt. In [80] the authors summarise recent studies in CACC, noting that in most systems the control laws are based either on classical control theory or Model Predictive Control. Their own experiments made use of four Nissan vehicles which were equipped with LIDAR-based ACC plus a 5.9-GHz DSRC communication system; in their case the control algorithm was designed using standard classical control methods. Figure 15 shows the effect of braking from the lead vehicle, which decelerates at $0.1 \mathrm{~g}$. In the upper plot (standard ACC) there are clear response delays as the vehicles further behind successively respond to the braking event from the front vehicle. There is a visible amplification in the acceleration response of vehicles following further behind, and this is the characteristic symptom of 'string instability'; indeed the fourth car bakes at $0.3 \mathrm{~g}$. For CACC (lower plot) the acceleration is no longer amplified - there is improved gap control and the system appears to be string-stable (at least in and around the test conditions). While such results have been known for a long time [58], the results clearly demonstrate the practical advantage of using wireless communication when ad-hoc platooning occurs among several ACC vehicles.
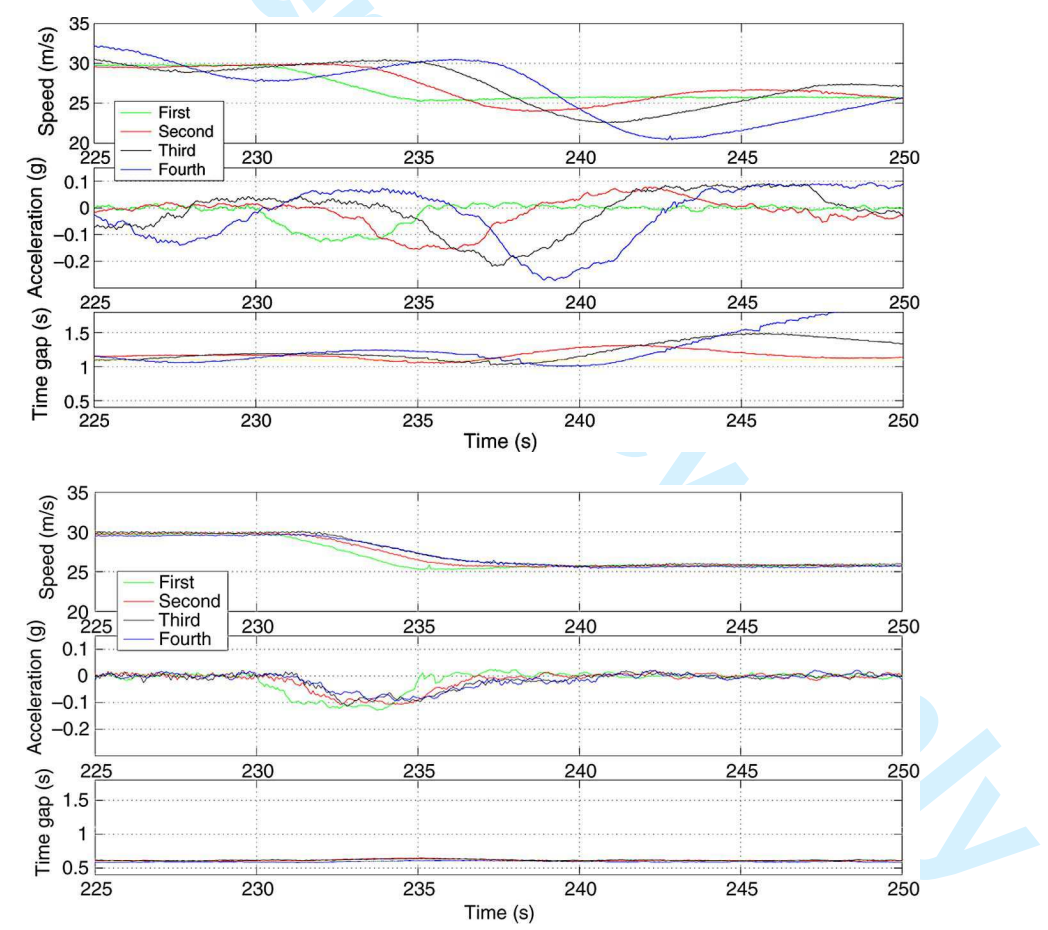

Figure 15. Real-world comparison of ACC (upper plot) and CACC (lower plot) responses due to braking of the lead vehicle. For $\mathrm{CACC}$ wireless communication enables a coordinated and string-stable response (from Milanes et. al $[80])$.

Further research will be needed however to show how similar performance can be achieved using distributed control among heterogeneous groups of ACC vehicles. One possible approach to address this challenge is to adopt a control architecture that makes 
use of infrastructure components to provide a coordinating function. We discuss this further in Subsection 6.3.

Convoys have also been proposed for fuel and carbon saving. The German national project KONVOI focused from 2005 to 2009 on the topic platoons of heavy trucks, in which vehicles follow a lead truck fully automatically with small gaps to improve aerodynamics. At the same time the small gaps and a digital transmission of driving parameter via $\mathrm{V} 2 \mathrm{~V}$ communication enable a reduction of needed road area and an improvement of traffic flow. Since the European projects PROMOTE CHAUFFEURI+II were the first research projects in the EU with this topic and these focused mainly on the technical feasibility, the project KONVOI studied the impact (driver acceptance, traffic flow and environment) as well as the legal and economic implication of platoons [81]. The KONVOI system was the first platoon system worldwide, which was tested in real traffic. The trucks were equipped with a V2V and V2I communication system, a mono camera as well as lidar and radar sensors. On the basis of the real traffic drives it could be shown, that a safe operation of platoons is possible. Researchers claimed that the trucks on the test track achieved some fuel-consumption savings even when they were driving at the 10-m gap between trucks; however, there was no fuel-consumption savings in the tests on the public highway because the trucks had to vary their speeds to respond to traffic conditions and other vehicles on the road [81]. As previously mentioned, the European project SARTRE [41] explored vehicle platooning with a view to reducing fuel economy, with a manually driven truck in the lead and trucks or light vehicles following under automated control. The study included tests on public highways [82] and results show fuel saving results up to $15 \%$, see Figure 16 .

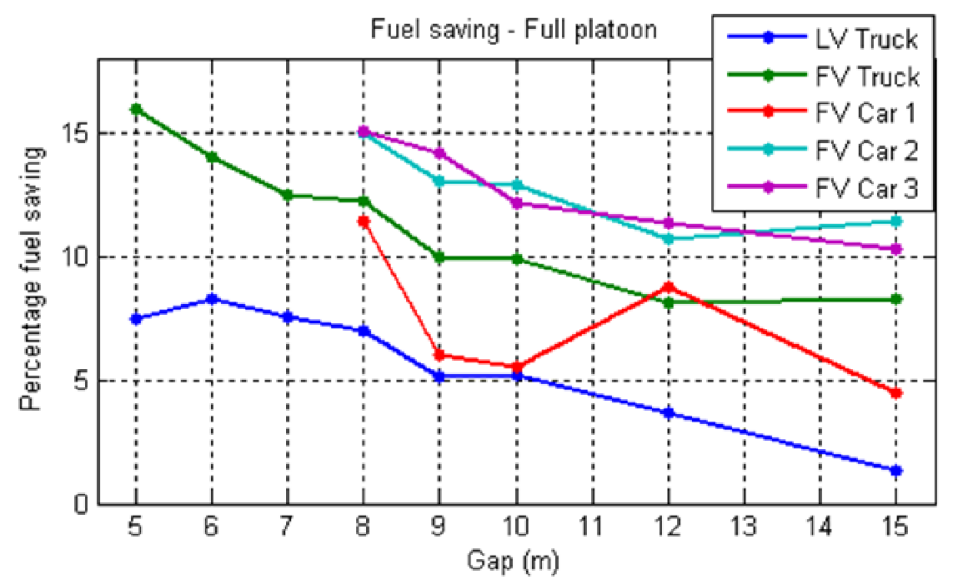

Figure 16. Fuel saving results from the SARTRE project: effect of inter-vehicle gap on fuel consumption reduction (from Chan $[82]$ ).

\subsection{Tactical Support from Smart Infrastructure}

In the above it is seen to be important to reduce the demands on tactical driving to enable higher levels of vehicle driving automation. Information from maps and from real-time infrastructure communication systems can improve efficiency and safety. For example, road preview can be useful when controlling hybrid powertrains. In [83] using 5 to 15 seconds of preview control for traffic conditions can lead to useful fuel saving of 1-2\%, 
which is also similar to the topographic preview analysis of [84]. While these may be only modest savings, in city driving or at higher levels of automation, it can be expected that infrastructure support would have greater benefits on journey times and fuel efficiency.

In Figure 17 we propose the concept of tactical support from the infrastructure. One example for this is connectivity to traffic light controller which adjust their patterns to upstream traffic flows, and communicate this to DSRC equipped vehicles [85]. Another case relates to Level 3 driving where infrastructure surveillance (e.g. on motorways) could help guarantee greater time horizons for safe automated driving - to confirm that the traffic flow ahead is stable for some distance and that there are no intrusions by animals or other hazards. The tactical support function might operate as a kind of 'ground traffic control' in communication with the automated driving system but not (as conceived here) with the human driver. According to this concept, a smart infrastructure provides tactical support to reduce the required intelligence for tactical driving, reducing the demands for on-board AI systems.

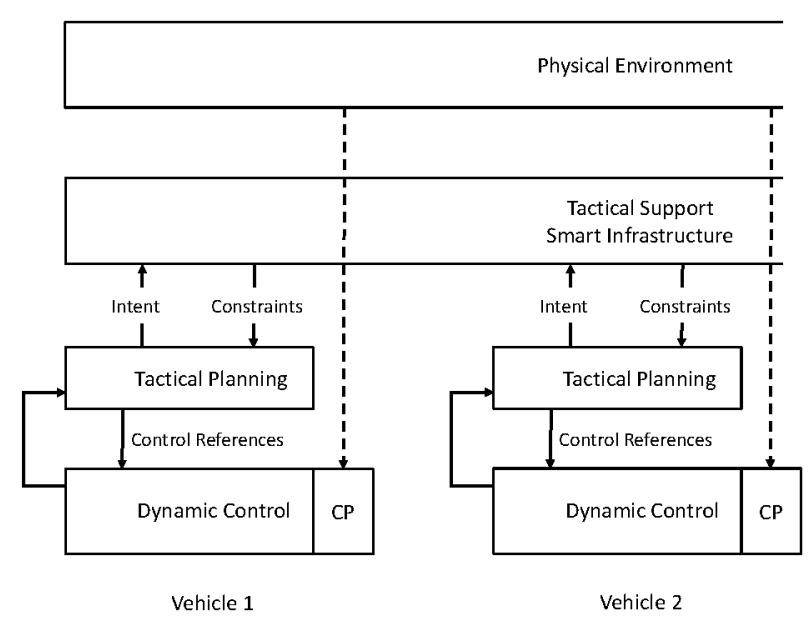

Figure 17. Tactical support from the infrastructure: an enhanced control architecture designed to support automated driving functions at all levels of automation.. CP denotes Collision Protection, responding directly to the physical environment

\subsection{Lateral control optimization}

For lane-keeping and lane changing the control demands are modest provided there is sufficient positioning accuracy within the lane(s) and that lane-level map positioning is achieved. As mentioned in Section 4, vision systems may be used to determine vehicle position relative to lane boundaries; in particular the previewed path error and local yaw angle error are needed; this is then sufficient to enable satisfactory lane tracking [86].

An alternative approach, avoiding the use of vision systems, comes from the California Partners for Advanced Transit and Highways (PATH) program which adopted a magnetic road reference system (permanent magnetic markers - Section 4). Without preview the (linear) control scheme is prone to instability, since with the "look down" characteristics 
of a front-bumper mounted magnetometer, the sensor-to-response dynamics have poorly damped open-loop poles and zeros, which worsen at higher speeds - hence even a small feedback gain generates instability in the root-locus plot [1]; in more colloquial terms, it is not possible to drive at speed by looking at the road beneath the car. To overcome this problem it was found sufficient to include a second rear-bumper mounted magnetometer, and use the combination of sensor measurements (plus vehicle inertial sensors) to estimate path curvature. This allows the lateral controller to provide "virtual preview" and hence a more acceptable controller. In this approach, preview is only effective up to a few car-lengths due to sensor noise and variations in path curvature [1].

In [87] another enhancement is included, this time to use tracking of a vehicle ahead to supplement one of the magnetometers if the second one is lost. LIDAR was assumed in this work, but a vision system could also be used.

Optimal control methods can also be used for lane-keeping and simple manoeuvres such as lane-changing. In either case it is standard to assume a target path and then track towards that path. The control problem is not difficult, at least as long as the relevant vehicle positioning and map data (or equivalent) is available to compute previewed path errors. Again, one may treat the control problem as one of "synthetic driver modeling" and many optimal and operational methods are available [88],[70],[89].

The challenge of defining a reference path - as well as the motivation to avoid this step - is even greater in the case of collision avoidance relative to a moving vehicle [90] In this work the scenario is relatively simpe - a lane change - and the motion of other vehicles are presumed to be fully known. It does however include cases where the other vehicle is accelerating or moving in a curved path, so the approach is both general and informative. The optimization uses a cost function in the form of a weighted sum of squares $J=\frac{1}{2} \int_{0}^{t_{f}}\left(y^{2}+w \dot{\delta}^{2}\right) d t$ which penalizes control action (steering angle velocity $\dot{\delta}$ ) and deviation from the pre-defined reference path $y$. The authors use formal optimal control (Pontryagin Minimum Principle) and a linear bicycle model. While this provides a reference for comparison of more implementable control algorithms, the path definition is outside of the optimization and the use of a linear vehicle model is not always plausible.

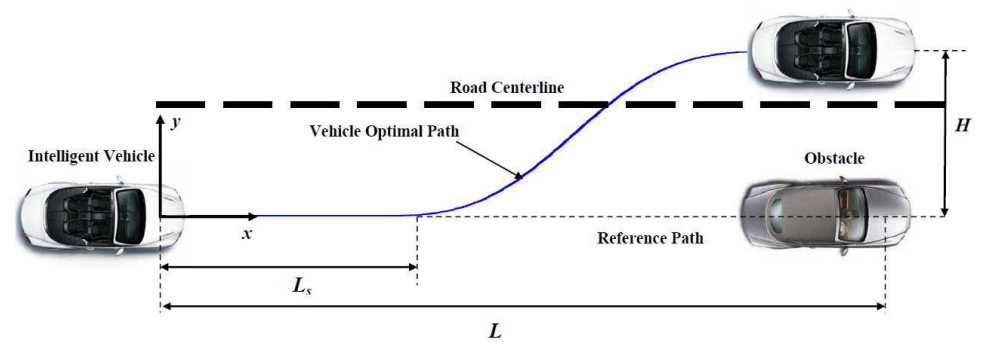

Figure 18. Path planning for collision avoidance relative to a moving obstacle (from Mashadi [90]).

\section{Naturalistic Driving Control}

There is a possible need for automated driving to exhibit naturalistic properties, e.g. to be 'comfortable', 'smooth' and 'natural'. Certainly, drivers and occupants are aware of the behaviour of automated driving systems and sensitive to sudden changes to behaviour. 
From an early driving studies with ACC [18] it was reported that drivers are sensitive to small and sudden deceleration events when speed is automated - the tendency is to look forward to see the source of the event. Of course, for collision protection systems, there is no expectation to have naturalistic and smooth dynamic behaviour.

Ride comfort in road vehicles is normally associated with vertical road inputs in the form of road unevenness transmitted through the suspension [91]. Accelerations arising from cornering and braking are normally under the control of a human driver, and hence any resulting discomfort is not normally seen as part of the vehicle dynamics. But in cases where no human driver is immediately responsible for speed and/or steering control it may be that ride comfort will become a more important consideration in the longitudinal and lateral degrees of freedom. Existing experience from ACC and LKA should inform the levels of acceleration and jerk that are normally acceptable, but it will be interesting to know whether passengers are indeed less forgiving of more fully automated driving systems.

If the design direction for automated driving systems is to emulate certain types of human driving, then there is much existing research to draw upon. The main characteristics of human driving were reviewed by Macadam in [70]; there are many limitations in human drivers! Firstly, human driver suffer from time delays: reaction times for visualbased response are at least around $200 \mathrm{~ms}$, sometimes considerably more; auditory and tactile response times may be shorter, at around $140 \mathrm{~ms}$, though increasing as the stimulus reduces to near detection thresholds. Secondly there are visual and other sensitivity limits; according to several authors (see in [70]) driving is "90\% visual"; though vague, the statement broadly implies that, although other sources of information are relevant, the most critical information comes from human vision. For a "naturalistic driving machine" this does not of course imply using cameras and machine vision as the primary input for control - rather it emphasizes the need that humans have for preview information - to compensate for their inherent response delays and provide a safety margin for collision avoidance.

Adequate preview is also needed to achieve smooth steering control [70] irrespective of time delay. The dynamic response of human drivers can be characterised by bandwidth or, more specifically, by the crossover frequency. It has been shown that within some frequency range, the forward-path transfer function for steering approximates to the form $C(s) G(s)=\frac{\omega_{c} e^{-s T}}{s}$. Here the input is the error in lateral lane deviation and the output is vehicle lane position (in response to steering control from the driver). The crossover frequency is defined by the condition that the forward-path transfer function has unit gain; with the 'crossover model' form shown, the amplitude reduces at a rate of $20 \mathrm{~dB}$ per decade, helping to ensure the relative stability of the closed-loop system. A typical crossover frequency for an attentive driver is around $\omega_{c} \simeq 4$ radians $/ \mathrm{s}$, a little over $0.5 \mathrm{~Hz}$ [70]. One would expect that an acceptable lane keeping controller should have a similar frequency bandwidth, even though the stability margins can be greater, give a reduced time delay.

The literature has many examples which attempt to model human steering and speed control behaviour [70]. A common approach is to formulate a control model (for lateral or longitudinal control as appropriate) and perform parameter estimation to represent actual driving behaviour [89]. Commonly used control formulations are classical control [86], linear preview optimal control [92] , [93] and Model Predictive Control [94], [95].

Data for parameter estimation and model validation is normally recorded from driving simulators or vehicle driving studies. More recently such research performed using data from naturalistic driving studies [96],[97]; in [97] a longitudinal driver model is formulated to include a range of noise signals and time delays designed to represent functional errors 
arising from the complexity of human behaviour - even though such complex behaviours are not directly represented in the model. One interesting aspect of this work is that the model can provide basic information about the effects of driver behaviour on crash outcomes; in [97] this was done using Monte-Carlo simulation, with random processes represented via a pseudo-random number generator.

A somewhat different approach is to look for detailed patterns in human driving, again as observed in naturalistic driving studies; here the drivers use instrumented vehicles to perform normal driving tasks, typically driving the same car for several weeks (or months or even longer). In [98] the authors propose a candidate error function that may be relevant to lateral control in highway driving, particularly for low-workload conditions and when there is no special reason for the driver to adhere to the lane center. This approach allows for driver models to be created based on real-world patterns of behaviour extracted from driving data. Validation is then needed, but it is a more stringent approach and has already yielded some interesting results. In [99] the authors determine that for steering control in lane-keeping, the driver tends to apply short correcting pulses that can be related to visually perceived tracking errors. A candidate model is presented and some validation and verification is made. However the authors show that there is much future work to be done in this area. Interestingly in [100] a similar pulse extraction is found, although the authors did not implement a computational model based on the findings.

As new generations of vehicles enjoy more sophisticated interfaces and control-level driver support (Section 3.1), there may be a need for further research into what becomes naturalistic with these new technical features.

\section{Automatic Control at the Limits of Friction}

In Section 6 the focus was on optimised and optimal automatic control for normal driving. Under more dynamic conditions, such as in collision avoidance, the need for optimal control is even more obvious - obtaining the best possible outcome given the limits of friction and available actuators. Vehicle control is challenging at the limits of handling but automated systems have the potential to safely negotiate difficult driving situations, where the human driver has neither the skills nor the same full range of actuators at his or her disposal.

In a similar but further developed approach for shared steering control a mediator is included as an internal part of the control algorithm that explicitly considers vehicle stability and boundary constraints as it attempts to track a precomputed trajectory; such a mediator can choose to violate short term stability constraints in order to avoid a collision [101], [102] and [103]. In [103] experiments are conducted with X1, a rearwheel drive electric vehicle, shown in Figure 19. An integrated GPS-INS system provides vehicle states in real-time, and a ruggedized i7 computer outputs controller commands at $100 \mathrm{~Hz}$ to the vehicles drive-by-wire system.

There is something of a conundrum regarding path planning (followed by path tracking). If a planned path is updated at each major computational time step, it cannot have any fundamental significance other than to simplify the control optimization problem to encode and represent the local constraints or objectives, for example to avoid collisions based on current vehicle states. On the other hand, if the planned path is not updated at each major time step then it surely has some fundamental significance - even given a set of possible initial vehicle states (including position) it is always the preferred path of motion for this initial set, even if when it is not even feasible! Except where there is 


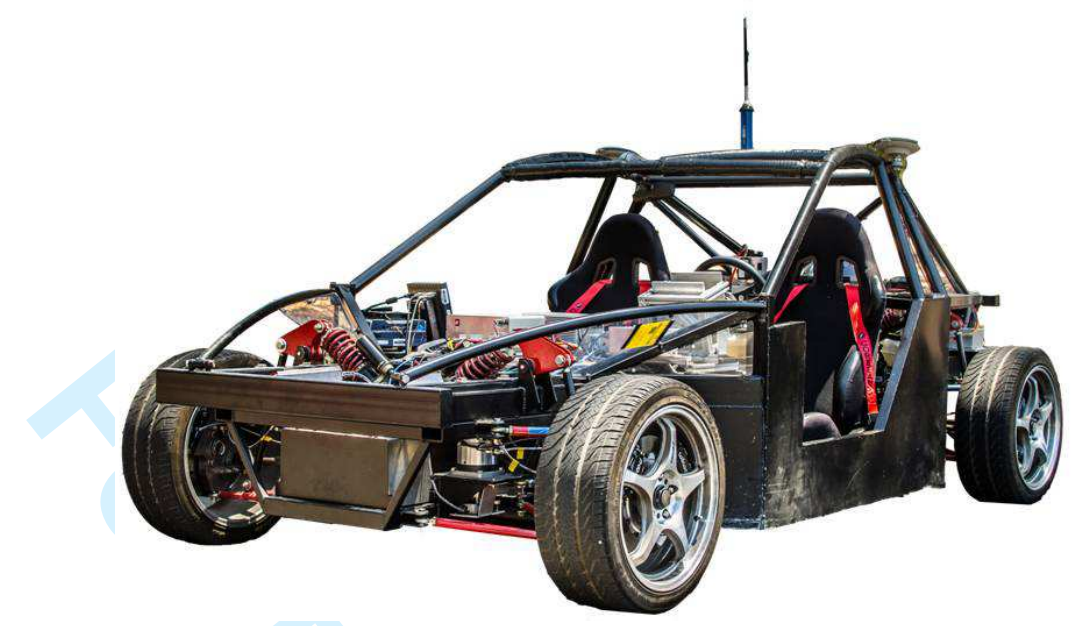

Figure 19. Steer-by-wire test vehicle X1 from Dynamic Design Lab, Stanford. (from Funke [103]).

a physical or other penalty for deviating from that path then surely this is incorrect a more fundamental optimal control solution would not generate such a path at all. Of course it is common to make simplifying assumptions when solving complex problems, but especially when friction limits are approached, the wasteful nature of tracking a somewhat arbitrary reference path should be clear. And in either of the above cases the fundamental need for a pre-planned path is not at all clear.

In [104] a completely different approach was taken - a control strategy was developed around the pre-definition of a vector field: a spatially distributed set of target velocities defined in the plane of the road, this being used as a reference for desired CG motion. In Figure 20 such a field is shown in the form of its integral curves (the field is tangent to the flow lines shown). In this case there is no reference path, but there is a stable trajectory that a (particle) would follow, if for example the road looped around in a closed circuit. And another path (in this case the center-line) was used in the construction of the vector field (a previewed point on the centreline was used to define the direction of the field). The method was applied to a double lane-change manoeuvre [105] in which the vector field was pre-optimised for the road geometry (but not any vehicle states), and results compared well with a full application of general nonlinear optimal control methods (Pontryagin Minimum Principle).

Further recent work has shown that a path reference is unnecessary for friction-limited optimal control problems, e.g. for wundersteer compensation using independent fourwheel braking [106] and for path control during a potential multiple event accident using automated braking and steering [107]. In the first case a mass centre acceleration vector reference is used; in the second case the two-point boundary value problem was simplified using Quasi-Linear Optimal Control (QLOC), and it was found that optimal path control is feasible even while a high degree of yaw instability persists after an initial collision. Another example, where particle-based optimisation was used in preference to a target path, is in a collision avoidance scenario [108].

This is not to say that path planning is never necessary - for example in [109] a discrete optimization process is invoked to decide the optimum (discretised) path - but this type of problem is more relevant to robots moving in unstructured environments than to vehicles moving on highways. Some discrete decision making is necessary (and is not discussed here), and for low-speed maneuvering it is perhaps always sensible to consider 
path planning as an important part of the optimal motion problem. But for frictionlimited handling dynamics it appears there are often superior approaches available.

\section{Conclusion and Future Developments}

The motivation for this review paper was to understand new challenges for vehicle system dynamics in the era when there are unparalleled increases in the variety and level of automation in road vehicles, particularly the automation of driving itself. We have seen two perspectives in the march towards increasing automation: the 'ABS perspective' with automation gradually evolving on the vehicle; and the 'DARPA perspective' which employs large arrays of sensors, combined with artificial intelligence, to replace the human driver and replicate the widest possible range of human driving activities. The reality for highway vehicles is likely to be a combination of the two, building new 'intelligent vehicle' capabilities on a foundation of well-established vehicle dynamic control practice.

One clear idea emerging from the foregoing is the need to simplify the driving environment and hence reduce the tactical driving requirements for highly automated driving modes, e.g. Level 4 where the driver is not required to recover control in the event of some fault or disruption.

Another idea that emerges is that full automation (Level 5) implies a system that performs more like a personalized rapid transit system - i.e. more like a flexible rail transport system but without the physical rails. This seems to imply the introduction of a centralised command and control centre to operate and monitor the system.

Level 3 driving automation (the driver is required to recover control) requires a time window for the human to recover situation awareness and control capability, and again a structured and predictable environment is needed to guarantee reasonable time windows 
- at least 2-3 seconds, preferably longer. In fact the separation between Levels 3 and 4 hinges on whether the event "unresponsive driver" is handled deterministically by the system (level 4 has a fully operational fail-safe mode) or stochastically (Level 3 would employ a risk reduction technique such as gradual stopping with hazard lights showing).

All levels of driving automation benefit from collision protection, and lessons learned from supporting human drivers (Level $0+$ active safety) will naturally support the evolution of driving automation. And with Level 4 automation being seriously contemplated (no human needed for fail-safe operation) the need to handle on-board system failures (e.g. steering motor faulty) must be fully handled without using driver mechanical controls, at least during the available automated driving modes. Hence electrical and electronic system redundancy should be sufficient to handle such conditions, and in that case why insist on retaining mechanical connections to the driver at all? The same system-level redundancy for control can operate when there is a fault during manual driving; the need to retain a mechanical link between the driver and the road wheels (or brake cylinders) is no longer clear. Hence it might be that the push towards high levels of automation both enables and is enabled by a move toward a fully drive-by-wire vehicle design.

Another, somewhat surprising, conclusion from this review is that mapping and localization, conceptually very simple problems, remain a challenge for driving automation. In the absence of a 'magic fix' in the near future, the emphasis is likely to be on developing high levels of automation in limited geographical areas, where enhanced maps can be created and maintained in an adequate manner. Enhanced infrastructure ("smart roads") would then develop hand-in-hand with this, likely including highway and traffic monitoring to reduce risk and exclude unexpected hazards.

In summary, the grand challenges for artificial intelligence - entirely replacing the human driver - is likely too great to be solved in the next few decades, except where the immediate driving environment can be simplified. This may be a fully revamped infrastructure (in local regions) or it could be achieved by novel means (like the SARTRE road train concept). And the overriding challenge will be to ensure human safety for the new systems, knowing that most existing highway safety evaluation is based on retrospective studies.

\section{References}

[1] Rajamani R, Tan HS, Law BK, Zhang WB. Demonstration of integrated longitudinal and lateral control for the operation of automated vehicles in platoons. Control Systems Technology, IEEE Transactions on. 2000;8(4):695-708.

[2] Kiencke U, Nielsen L. Automotive Control Systems. Springer; 2005.

[3] van Zanten A. Bosch ESP systems: 5 years of experience. SAE 2000-01-1633. 2000;.

[4] van Zanten A. Evolution of electronic control systems for improving the vehicle dynamic behavior. In: Proceedings of the 6th International Symposium on Advanced Vehicle Control; 2002. p. 1-9.

[5] Thrun S, Montemerlo M, Dahlkamp H, Stavens D, Aron A, Diebel J, Fong P, Gale J, Halpenny M, Hoffmann G, Lau K, Oakley C, Palatucci M, Pratt V, Stang P, Strohband S, Dupont C, Jendrossek LE, Koelen C, Markey C, Rummel C, Niekerk J, Jensen E, Alessandrini P, Bradski G, Davies B, Ettinger S, Kaehler A, Nefian A, Mahoney P. Stanley: The Robot That Won the DARPA Grand Challenge. The 2005 DARPA Grand Challenge, Springer Tracts in Advanced Robotics. 2007; $36: 1$.

[6] Buehler M, Iagnemma K, Singh S. The darpa urban challenge: autonomous vehicles in city traffic. Vol. 56. Springer Science \& Business Media; 2009.

[7] National Academy of Engineering. Frontiers of Engineering: Reports on Leading-Edge Engineering from the 2014 Symposium. Washington, DC: The National Academies Press; 2015. 
[8] Michon JA. A critical view of driver behavior models: what do we know, what should we do? Springer; 1986.

[9] Shinar D, Tractinsky N, Compton R. Effects of practice, age, and task demands, on interference from a phone task while driving. Accident Analysis \& Prevention. 2005;37(2):315-326.

[10] Young K, Regan M, Hammer M. Driver distraction: A review of the literature. Distracted driving Sydney, NSW: Australasian College of Road Safety. 2007;:379-405.

[11] Wilson FA, Stimpson JP. Trends in fatalities from distracted driving in the united states, 1999 to 2008. American Journal of Public Health. 2010;100(11):2213-2219.

[12] Regan M, Young K. Use of Manual Speed Alerting and Cruise Control Devices by Drivers in New South Wales. Accident Research Centre, Monash University; 2004. Report no.:.

[13] Vollrath M, Schleicher S, Gelau C. The influence of Cruise Control and Adaptive Cruise Control on driving behaviour-A driving simulator study. Accident Analysis \& Prevention. 2011;43(3).

[14] Fancher P, Bareket Z. Evaluating Headway Control Using Range Versus Range-Rate Relationships. Vehicle System Dynamics. 1994;23(1):575-596.

[15] Watanabe T, Kishimoto N, Hayafune K, Yamada K. Development of an Intelligent Cruise Control System. In: Proceedings of the World Congress on Intelligent Transport Systems; 1995. p. 12291235.

[16] Rajamani R. Vehicle Dynamics and Control. Springer; 2006.

[17] Mastinu G, Ploechl M, editors. Road and Off-Road Vehicle System Dynamics Handbook. CRC Press; 2014.

[18] Fancher P, Ervin R, Sayer J, Hagan M, Bogard S, Bareket Z, Mefford M, Haugen J. Intelligent cruise control field operational test. Final report. University of Michigan Transportation Research Institute Report; 1997. Report No.: UMTRI-97-11.

[19] Rakha H, Hankey J, Patterson A, Van Aerde M. Field Evaluation of Safety Impacts of Adaptive Cruise Control. ITS Journal. 2001;6:225-259.

[20] Inagaki T. Adaptive Automation: Sharing and Trading of Control; 2003. Chapter Chapter 8 of the Handbook of Cognitive Task Design; p. 147-169.

[21] Seppelt B, Lees M, Lee J. Driver Distraction and Reliance: Adaptive Cruise Control in the Context of Sensor Reliability and Algorithm Limits. In: Proceedings of the 3rd International Driving Symposium on Human Factors in Driver Assessment, Training, and Vehicle Design.; June 27-30. Rockport, Maine; 2005.

[22] Grover C, Knight I, Okoro F, Simmons I, Couper G, Massie P, Smith B. Automated Emergency Braking Systems: Technical requirements, costs and benefits. EC; 2008. Report no.:

[23] Distner M, Bengtsson M, Broberg T, Jakobsson L. City Safety - A System Addressing Rear-End Collisions at Low Speeds. In: ESV 2009; 2009.

[24] Isaksson-Hellman I, Lindman M. The Effect of a Low-Speed Automatic Brake System Estimated From Real Life Data. In: ???; 2012.

[25] Ackermann J, Bunte T, Odenthal D. Advantages Of Active Steering For Vehicle Dynamics Control. 1999;.

[26] Chai Y, Kimura T, Igarashi K. Contribution for Vehicle Dynamics with a Steering System which Controls Tire Angles and Steering Force Independently. In: 12th International Symposium on Advanced Vehicle Control (AVED'14); September 22-26; 2014.

[27] Shibahata Y, Shimada K, Tomari T. Improvement of Vehicle Maneuverability by Direct Yaw Moment Control. Vehicle System Dynamics. 1993;.

[28] Hancock MJ, Williams RA, Fina E, Best MC. Yaw motion control via active differentials. Trans Inst Meas Control. 2007 Jun;29(2):137-158.

[29] Tomari T, Mori A, Shibahata Y. Development of SH-AWD Based on DYC (Direct Yaw Control) Concept. In: The 8th International Symposium on Advanced Vehicle Control; 2006. p. 611-616.

[30] Pohl J, Ekmark J. Development of a haptic intervention system for unintended lane departure. $2003 ;$

[31] Katzourakis D, Olsson C, Nedad L, Lidberg M. Driver Steering Override Strategies for Steering Based Active Safety Systems. In: FAST-zero 13, Second International Symposium on Future Active Safety Technology Toward zero traffic accidents. Nagoya, Japan; 2013.

[32] Gordon T, Sardar H, Blower D, Ljung Aust M, Bareket Z, Barnes M, Blankespoor A, IsakssonHellman I, Ivarsson J, Juhas B, et al. Advanced crash avoidance technologies (acat) program-final report of the volvo-ford-umtri project: safety impact methodology for lane departure warningmethod development and estimation of benefits; 2010. Report no.:

[33] Taxonomy and Definitions for Terms Related to On-Road Motor Vehicle Automated Driving Systems. 2014 January. 
[34] Sharp RS, Peng H. Vehicle dynamics applications of optimal control theory. Vehicle System Dynamics. 2011;49(7):1073-1111.

[35] Tagesson K. Truck Steering System and Driver Interaction: Dissertation for Licentiate [dissertation]. Chalmers University of Technology; 2014.

[36] Pfeffer P. Interaction of Vehicle and Steering System Regarding On-Centre Handling [dissertation]. University of Bath; 2006.

[37] Ciarla V, Cahouet V, Canudas de Wit C, Quaine F. Genesis of booster curves in electric power assistance steering systems. In: The 15th International IEEE Conference on Intelligent Transportation Systems; 2012. p. 1345-1350.

[38] Klomp M. DRIVE MECHANISM FOR SELECTIVELY SWITCHING A DRIVE BETWEEN PROPULSION AND TORQUE VECTORING. 2011.

[39] Stanton NA, Marsden P. From fly-by-wire to drive-by-wire: Safety implications of automation in vehicles. Safety Science. 1996;24(1):35 - 49.

[40] Shladover S, Desoer C, Hedrick K, Tomizuka M, Walrand J, Zhang WB, McMahon D, Peng H, Sheikholeslam S, McKeown N. Automatic Vehicle Control Developments in the PATH Program. IEEE Transactions on Vehicular Technology. 1991 February;40(1):114-130.

[41] Bergenhem C, Huang Q, Benmimoun A, Robinson T. Challenges of Platooning on Public Motorways. In: 17th World Congress and Exhibition on Intelligent Transport Systems and Services (ITS 2010); 2010. p. 1-12.

[42] Dodson S. Welcome to the transport of tomorrow. The Guardian. 2007 October 10;.

[43] Why Has Masdar Personal Rapid Transit (PRT) Been Scaled Back? In: Conference on Personal Rapid Transit; September 21-23rd; 2010.

[44] Vincent J. Milton keynes introducing driverless public transport pods by 2017. The Independent. 2013 November 6;.

[45] Falcone P, Borrelli F, Tseng E, Hrovat D. On Low Complexity Predictive Approaches to Control of Autonomous Vehicles. Springer-Verlag; 2010. Chapter Chapter 13; p. 195-210.

[46] Tsang SH, Hall PS, Hoare EG, Clarke NJ. Advance path measurement for automotive radar applications. Intelligent Transportation Systems, IEEE Transactions on. 2006;7(3):273-281.

[47] Leonard J, Barrett D, How J, Teller S, Antone M, Campbell S, Epstein A, Fiore G, Fletcher L, Frazzoli E, et al. Team mit urban challenge technical report. 2007;

[48] Mei C, Sibley G, Cummins M, Newman P, Reid I. Rslam: A system for large-scale mapping in constant-time using stereo. International journal of computer vision. 2011;94(2):198-214.

[49] Urmson C, Ragusa C, Ray D, Anhalt J, Bartz D, Galatali T, Gutierrez A, Johnston J, Harbaugh S, Messner W, et al. A robust approach to high-speed navigation for unrehearsed desert terrain. Journal of Field Robotics. 2006;23(8):467-508.

[50] Toledo-Moreo R, Bétaille D, Peyret F. Lane-level integrity provision for navigation and map matching with gnss, dead reckoning, and enhanced maps. Intelligent Transportation Systems, IEEE Transactions on. 2010;11(1):100-112.

[51] Joshi A, James M. Generation of accurate lane-level maps from coarse prior maps and lidar. Intelligent Transportation Systems Magazine, IEEE. 2015;7(1):19-29.

[52] Haklay M, Weber P. Openstreetmap: User-generated street maps. Pervasive Computing, IEEE. 2008;7(4):12-18.

[53] Graefe G, Caspary W, Heister H, Klemm J, Sever M. The road data acquisition system mosesdetermination and accuracy of trajectory data gained with the applanix pos/lv. In: Proceedings, The Third International Mobile Mapping Symposium, Cairo, Egypt, January. Citeseer; 2001. p. $3-5$.

[54] Krygier J, Wood D. Making maps, second edition: A visual guide to map design for gis. Guilford Publications; 2011; Available from: https://books.google.co.uk/books?id=jr2fZEitOnkC.

[55] Cheng W, Cheng X, Song M, Chen B, Zhao WW. On the design and deployment of rfid assisted navigation systems for vanets. Parallel and Distributed Systems, IEEE Transactions on. 2012; 23(7):1267-1274.

[56] Misener JA, Sengupta R, Ahern K, Datta Gupta S, Dickey S, Kuhn T, Lian T, Manasseh C, Nelson D, Rezai S, et al. Vii california: Development and deployment proof of concept and groupenabled mobility and safety (gems); 2010. Report no..:

[57] Alam N, Balaei AT, Dempster AG. An instantaneous lane-level positioning using dsrc carrier frequency offset. Intelligent Transportation Systems, IEEE Transactions on. 2012;13(4):1566-1575.

[58] Shladover S. Review of the state of the development of development of advanced vehicle control systems (AVCS). Vehicle System Dynamics. 1995;(24):551-595.

[59] Distner M, Bengtsson M, Broberg T, Jakobsson L. City safetya system addressing rear-end colli- 
sions at low speeds. In: Proc. 21st International Technical Conference on the Enhanced Safety of Vehicles; 09-0371; 2009.

[60] Hamdane H, Serre T, Anderson R, Masson C, Yerpez J. Description of pedestrian crashes in accordance with characteristics of active safety systems. In: IRCOBI Conference Proceedings; 2014.

[61] Coelingh E, Eidehall A, Bengtsson M. Collision warning with full auto brake and pedestrian detection-a practical example of automatic emergency braking. In: Intelligent Transportation Systems (ITSC), 2010 13th International IEEE Conference on. IEEE; 2010. p. 155-160.

[62] Chun J, Lee I, Park G, Seo J, Choi S, Han SH. Efficacy of haptic blind spot warnings applied through a steering wheel or a seatbelt. Transportation Research Part F: Traffic Psychology and Behaviour. 2013;21:231-241.

[63] Glazduri V. An investigation of the potential safety benefits of vehicle backup proximity sensors. In: Proceedings of the International Technical Conference on Enhanced Safety Vehicles; Vol. 1; 2005. p. 2.

[64] Habibovic A. Analyzing real-world data to promote development of active safety systems that reduce car-to-vulnerable road user accidents. Chalmers University of Technology; 2012.

[65] Dept of Transportation (US) NHTSAN. Traffic Safety Facts 2012.; 2012. Report no.:.

[66] Da Lio M, Biral F, Bertolazzi E, Galvani M, Bosetti P, Windridge D, Saroldi A, Tango F. Artificial co-drivers as a universal enabling technology for future intelligent vehicles and transportation systems. IEEE Transactions on Intelligent Transportation Systems. 2015;16(1):244-263.

[67] Flemisch F, Nashashibi F, Rauch N, Schieben A, Glaser S, Temme G, Resende P, Vanholme B, Löper C, Thomaidis G, et al. Towards highly automated driving: Intermediate report on the haveitjoint system. In: 3rd European Road Transport Research Arena, TRA 2010; 2010.

[68] Rizzi M, Kullgren A, Tingvall C. The injury crash reduction of low-speed autonomous emergency braking (aeb) on passenger cars. In: IRCOBI Conference Proceedings; 2014.

[69] More good news about crash avoidance: Volvo city safety reduces crashes. ????;

[70] Macadam CC. Understanding and modeling the human driver. Vehicle System Dynamics. 2003; 40(1-3):101-134.

[71] Li SE, Peng H, Li K, Wang J. Minimum fuel control strategy in automated car-following scenarios. Vehicular Technology, IEEE Transactions on. 2012;61(3):998-1007.

[72] Suthaputchakun C, Sun Z, Dianati M. Applications of vehicular communications for reducing fuel consumption and co 2 emission: the state of the art and research challenges. Communications Magazine, IEEE. 2012;50(12):108-115.

[73] Guzolek J, Koch E. Real-time route planning in road networks. In: Vehicle Navigation and Information Systems Conference, 1989. Conference Record. IEEE; 1989. p. 165-169.

[74] Brockfeld E, Barlovic R, Schadschneider A, Schreckenberg M. Optimizing traffic lights in a cellular automaton model for city traffic. Physical Review E. 2001;64(5):056132.

[75] Xu Y, Zhang Y, Liu M. Multiagent based decentralized traffic light control for large urban transportation system. Mathematical Problems in Engineering. 2014;2014.

[76] Garcia-Nieto J, Olivera AC, Alba E. Optimal cycle program of traffic lights with particle swarm optimization. Evolutionary Computation, IEEE Transactions on. 2013;17(6):823-839.

[77] Ioannou P, Wang Y, Chang H. Integrated roadway/adaptive cruise control system: Safety, performance, environmental and near term deployment considerations. California PATH Program, Institute of Transportation Studies, University of California at Berkeley; 2007.

[78] Reghelin R, Arruda L. Optimizing travel time of intelligent vehicles in any highway configuration. Advances in Transportation Studies. 2014;(32).

[79] Pérez J, Milanés V, Godoy J, Villagra J, Onieva E. Cooperative controllers for highways based on human experience. Expert Systems with Applications. 2013;40(4):1024-1033.

[80] Milanés V, Shladover SE, Spring J, Nowakowski C, Kawazoe H, Nakamura M. Cooperative adaptive cruise control in real traffic situations. Intelligent Transportation Systems, IEEE Transactions on. 2014;15(1):296-305.

[81] US Department of Transportation. Recent International Activity in Cooperative VehicleHighway Automation Systems. 2012;

[82] Chan E. Sartre automated platooning vehicles. In: Transport Research Arena (TRA) 5th Conference: Transport Solutions from Research to Deployment; 2014.

[83] Kim TS, Manzie C, Sharma R. Model predictive control of velocity and torque split in a parallel hybrid vehicle. In: Systems, Man and Cybernetics, 2009. SMC 2009. IEEE International Conference on. IEEE; 2009. p. 2014-2019.

[84] Johannesson L, Egardt B. A novel algorithm for predictive control of parallel hybrid powertrains based on dynamic programming. In: Advances in Automotive Control; Vol. 5; 2007. p. 343-350. 
[85] Ferreira M, Fernandes R, Conceição H, Viriyasitavat W, Tonguz OK. Self-organized traffic control. In: Proceedings of the seventh ACM international workshop on VehiculAr InterNETworking. ACM; 2010. p. 85-90.

[86] Weir DH, McRuer DT. Dynamics of driver vehicle steering control. Automatica. 1970;6(1):87-98.

[87] Lu G, Tomizuka M. Vehicle following as backup control schemes for magnet-magnetometer-based lateral guidance. Control Systems Technology, IEEE Transactions on. 2005;13(2):274-285.

[88] Guo K, Guan H. Modelling of driver/vehicle directional control system. Vehicle System Dynamics. 1993;22(3-4):141-184.

[89] Plöchl M, Edelmann J. Driver models in automobile dynamics application. Vehicle System Dynamics. 2007;45(7-8):699-741.

[90] Mashadi B, Majidi M. Global optimal path planning of an autonomous vehicle for overtaking a moving obstacle. Latin American Journal of Solids and Structures. 2014;11(14):2555-2572.

[91] Griffin MJ. Discomfort from feeling vehicle vibration. Vehicle System Dynamics. 2007;45(7-8):679698.

[92] MacAdam CC. An optimal preview control for linear systems. Journal of dynamic systems, measurement, and control. 1980;102(3):188-190.

[93] Odhams A, Cole D. Application of linear preview control to modelling human steering control. Proceedings of the Institution of Mechanical Engineers, Part D: Journal of Automobile Engineering. 2009;223(7):835-853.

[94] Prokop G. Modeling human vehicle driving by model predictive online optimization. Vehicle System Dynamics. 2001;35(1):19-53.

[95] Keen SD, Cole DJ. Steering control using model predictive control and multiple internal models. In: Proceedings of the 8th International Symposium on Automotive Control (AVEC 2006); 2006. p. 599-604.

[96] Lee K, Peng H. Identification and verification of a longitudinal human driving model for collision warning and avoidance systems. International journal of vehicle autonomous systems. 2004;2(1):317.

[97] Yang HH, Peng H. Development and evaluation of collision warning/collision avoidance algorithms using an errable driver model. Vehicle System Dynamics. 2010;48(S1):525-535.

[98] Gordon T, Blankespoor A, Barnes B, Blower D, Green P, Kostyniuk L. Yaw rate error-a dynamic measure of lane keeping control performance for the retrospective analysis of naturalistic driving data. In: 21st International Technical Conference on the Enhanced Safety of Vehicles, Stuttgart, Germany; 2009. p. 09-0326.

[99] Gordon T, Srinivasan K. Modeling human lane keeping control in highway driving with validation by naturalistic data. In: Systems, Man and Cybernetics (SMC), 2014 IEEE International Conference on. IEEE; 2014. p. 2507-2512.

[100] Benderius O, Markkula G. Evidence for a fundamental property of steering. In: Proceedings of the Human Factors and Ergonomics Society Annual Meeting; Vol. 58. SAGE Publications; 2014. p. $884-888$.

[101] Beal C. Applications of Model Predictive Control to Vehicle Dynamics for Active Safety and Stability [dissertation]. Stanford University; 2011.

[102] Erlien S, Susumu F, Gerdes C. Safe Driving Envelopes for Shared Control of Ground Vehicles. In: 7th IFAC Symposium on Advances in Automotive Control; 2010.

[103] Funke J, Brown M, Erlien S, Gerdes J. Prioritizing Collision Avoidance and Vehicle Stabilization for Autonomous Vehicles. To be submitted. 2015;

[104] Gordon T, Best MC, Dixon P. An automated driver based on convergent vector fields. Proceedings of the Institution of Mechanical Engineers, Part D: Journal of Automobile Engineering. 2002; 216(4):329-347.

[105] Gordon T, Best MC. On the synthesis of driver inputs for the simulation of closed-loop handling manoeuvres. International Journal of Vehicle Design. 2006;40(1):52-76.

[106] Klomp M, Lidberg M, Gordon T. On optimal recovery from terminal understeer. Proceedings of the Institution of Mechanical Engineers, Part D: Journal of Automobile Engineering. 2014; .

[107] Yang D, Gordon TJ, Jacobson B, Jonasson M. Quasi-Linear Optimal Path Controller Applied to Post Impact Vehicle Dynamics. submitted for publication in IEEE Transactions on Intelligent Transportation Systems. 2011;

[108] Moshchuk N, Chen SK, Zagorski C, Chatterjee A. Optimal braking and steering control for active safety. In: Intelligent Transportation Systems (ITSC), 2012 15th International IEEE Conference on. IEEE; 2012. p. 1741-1746.

[109] Rashid AT, Ali AA, Frasca M, Fortuna L. Path planning with obstacle avoidance based on visibility 
1

2

3

4

5

6

7

8

9

10

11

12

13

14

15

16

17

18

19

20

21

22

23

24

25

26

27

28

29

30

31

32

33

34

35

36

37

38

39

40

41

42

43

44

45

46

47

48

49

50

51

52

53

54

55

56

57

58

59

60

binary tree algorithm. Robotics and Autonomous Systems. 2013;61(12):1440-1449. 
To appear in Vehicle System Dynamics Vol. 00, No. 00, Month 20XX, 1-38

\title{
Automated Driving and Autonomous Functions on Road Vehicles
}

\author{
T.J. Gordon ${ }^{\mathrm{a} *}$, M. Lidberg $^{\mathrm{b}}$ \\ ${ }^{\mathrm{a}}$ School of Engineering, University of Lincoln, LN6 7TS, Lincoln, UK; ${ }^{\mathrm{b}}$ Department of Applied \\ Mechanics, Chalmers University of Technology, SE-412 96, Gothenburg, Sweden \\ (Received day-month-year.)
}

\begin{abstract}
In recent years, road vehicle automation has become an important and popular topic for research and development in both academic and industrial spheres. New developments have received extensive coverage in the popular press, and it may be said that the topic has captured the public imagination. Indeed, the topic has generated interest across a wide range of academic, industry and governmental communities, well beyond vehicle engineering; these include computer science, transportation, urban planning, legal, social science and psychology. While this follows a similar surge of interest - and subsequent hiatus - of Automated Highway Systems in the 1990's, the current level of interest is substantially greater, and current expectations are high. It is common to frame the new technologies under the banner of "self-driving cars" - robotic systems potentially taking over the entire role of the human driver, a capability that does not fully exist at present. However, this single vision leads one to ignore the existing range of automated systems that are both feasible and useful. Recent developments are underpinned by substantial and long-term trends in "computerisation" of the automobile, with developments in sensors, actuators and control technologies to spur the new developments in both industry and academia. In this paper we review the evolution of the intelligent vehicle and the supporting technologies with a focus on the progress and key challenges for vehicle system dynamics. A number of relevant themes around driving automation are explored in this article, with special focus on those most relevant to the underlying vehicle system dynamics. One conclusion is that increased precision is needed in sensing and controlling vehicle motions, a trend that can mimic that of the aerospace industry, and similarly benefit from increased use of redundant by-wire actuators.
\end{abstract}

Keywords: self-driving cars, vehicle automation, intelligent control, active safety, collision avoidance, vehicle sensors, driver-vehicle interaction.

\section{Introduction}

In the past, cars and trucks could be considered as complex mechanical systems, but since the 1980's they have become increasingly sophisticated mechatronic systems, fitted with many actuators, sensors and electronic control units. From early beginnings with fuel control, the level and authority of electronic control systems on vehicles has become increasingly sophisticated, including automated braking systems which operate independently of the driver to avoid collisions, automated steering for parking (capable of manoeuvring into smaller parking spaces than the average human driver), and automated driving (speed and steering) systems for use at low speed in traffic jams.

\footnotetext{
*Corresponding author. Email: tgordon@lincoln.ac.uk
} 
Several companies including Google, Volvo, Tesla and Daimler have been demonstrating fully self-driving vehicles that use computers rather than humans to do the driving. For safety reasons, these driverless or autonomous vehicles have test engineers closely supervising their progress. There are, however, intentions to make self-driving cars available to the general public. The US states of Nevada and California have introduced legislation to allow testing of self-driving cars on their highways, and the UK and German government recently announced similar initiatives.

Motivation for these developments are centred around a broad "technological fix" agenda - expanding human mobility, increasing travel efficiency in terms of time and fuel, freeing up the connected traveller from the demands of routine driving, and potentially improving safety. It is common to read statements like "over $90 \%$ of highway accidents occur due to driver-related human errors" [1], but we should not fall into the trap of concluding that automation implies a safety benefit - in the future we could be saying "over $90 \%$ of highway accidents are due to computer error".

Changing the way we drive road vehicles in a fundamental way offers many opportunities, and in this paper we explore the intersection of recent developments in this field with the fundamental engineering perspectives of vehicle system dynamics. In some scenarios the whole framework of road vehicle dynamics may change, so in the following we will evaluate these developments in the context of a long line of technological advances, much of which has arisen from or been influenced by the research outputs of the International Association of Vehicle System Dynamics.

Ceding complete control of cars to computers is just one end of a spectrum of automation concepts for road vehicles; there exists a growing portfolio of more limited but useful capabilities, particularly in areas of driver assistance and active safety.

An early example of the technologies needed for self-driving vehicles is the antilock braking system (ABS) [2]. Developed in the 1980s, ABS technology monitors wheel speeds and controls brake pressures to prevent wheels from locking up under hard braking. ABS may not be considered particularly smart these days, but its sensors and actuators provided a gateway towards more sophisticated systems. For example, in electronic stability control (ESC) [3], [4], an electronic control unit intervenes by using the brakes to correct for loss of stability, a significant risk on wet or icy roads. Compared with ABS, ESC interacts with the driver more conspicuously, correcting the way the car moves via feedback control: ESC compares actual with expected vehicle motions, and prevents the car from spinning dangerously or slipping sideways by the application of correcting yaw moments. Taken together, ESC and electronic engine control make it relatively simple and affordable to use engine and brakes to control a vehicle's speed, which is a suitable starting point for at least partially automating the driving process.

The above "ABS perspective" represents a particular 'entry route' to vehicle automation, focused around the traditional automotive companies and suppliers, building generation-by-generation on new actuators, sensors and interfaces. An alternative route has emerged from two competitive challenges, organized by the Defense Advanced Research Projects Agency (DARPA) in the United States during 2004-2007 [5], [6], [7]. The DARPA Grand Challenge involved autonomous driving across desert tracks (car vs. terrain) while the subsequent DARPA Urban Challenge introduced traffic conflicts via a simulated city scenario. While sensing and mapping capabilities in these challenges were far from what is economically feasible with current production cars, they do illuminate what is possible with current technologies when production costs are ignored. And, unlike the ABS/ESC developments, the focus was on computation and information processing, more than on the underlying vehicle dynamics and control systems.

Hence, from this 'DARPA perspective', the interesting challenges are mostly related to 


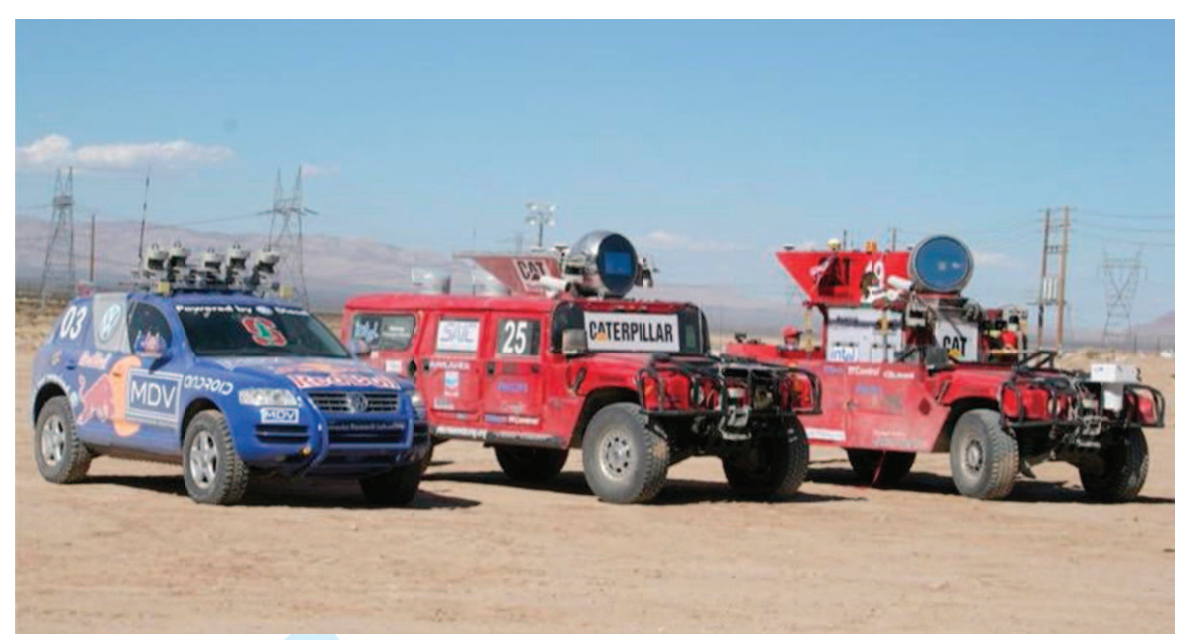

Figure 1. The top three finishers in the 2005 DARPA Grand Challenge; vehicles from Stanford University (left), and Carnegie Mellon University (centre and right). Reproduced from [7].

sophisticated optical systems and image processing, high powered computation, precision location and mapping, data fusion and (artificial) intelligence. These capabilities, so clearly an existing part of what the human driver achieves, occupy the "tactical space" of driving. This is part of a well-established conceptual model of human driving that is worthy of closer inspection given the new trends in vehicle automation. The well-accepted conceptual model of Michon [8] states that there are three levels of human activity in traditional manual driving: strategic (planning), tactical (manoeuvring and shortterm decision making) and control (actuation of steering etc. typically using localised feedback).

In Figure 2, we formulate that the Strategic, Tactical and Control driving processes may involve automated systems, in full or in part. Note that there are different timescales of operation between these levels, ranging from minutes/hours/days at the strategic level, to a few seconds for manoeuvring, and then down to a few tens of milliseconds at the control level [8]. These timings are inherited from the needs of vehicle motion control itself, so there is little reason to expect them to change significantly as a result of automation.

At the upper level, strategic decisions affect the general planning stage of a trip, including goals, route, and possibly choice of transportation mode, based on preferences, costs and perceived risks. General considerations about transport and mobility may influence these decisions, as indeed may information gathered before or during a trip. In the modern "connected" world, with near-universal access to real-time information, it is common for general plans to evolve over time. In Figure 2 the Environmental Inputs are assumed to be directly perceived (e.g. weather conditions, visually) while External processes are considered to be the result of separate agencies, e.g. public information services. Note: we use the term driver throughout, meaning the person in the vehicle most likely to carry out actions in response to some event. For discussion, we exclude the case of a vehicle without any human occupant.

The Tactical Level is where the driver and/or automated systems plan manoeuvres within traffic, decide to change lanes, slow for a curve, prepare to take a motorway exit lane etc. In Figure 2 these actions - plus supporting information processing - are represented by Tactical Level block. Again, directly relevant information comes from the left (road geometry, traffic movements etc.) while agency-sourced information comes from the right. According to [8] the actions are in the form of "controlled action patterns" from 
External processes

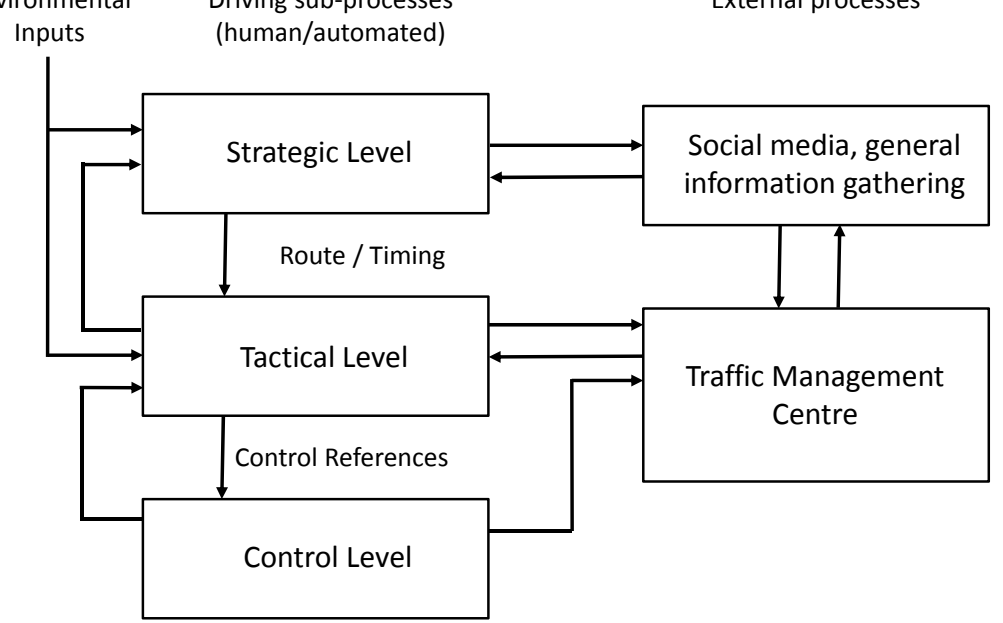

Figure 2. Hierarchical structure of the driving task including attention switching (after Michon [8]).

the driver, implying that conscious decisions or plans are made based on the evolving road and traffic situation, or that previous experience is used to decide the timing and amplitude of lower level control actions. For (partially or fully) automated systems, this layer may for example determine feasible or desirable vehicle trajectories that can then be passed to the control level.

Here we assume that the tactical level block is purely concerned with the core driving process; so, to represent the common behaviour of attention switching to non-driving activities (a typical and serious safety concern) [9], [10], [11] this is shown as a secondary process of attention control - visual, cognitive or both. While such attention switching is already considered important in the safety of manual driving, it is worth further emphasising its role in the case of partial or fully automated driving, as it strongly influences the availability of the driver to participate in control actions.

As well as human driver attention, we allow for the activities of external agencies, for example a traffic management centre which monitors traffic conditions and provides driving-relevant information to the human driver and/or any automated systems. And it may be that such information influences the reference information being passed down to the control level (e.g. ice warnings influence the action threshold for a collision avoidance system). As mentioned, from the "DARPA perspective" the major developments have been rooted in the tactical level.

The control level responds to reference information coming from the tactical block, so rather than "seeing" lane markers the control layer would "see" the need to increase yaw rate or decrease speed according to some objective reference information. The dividing line between the tactical and control levels is not universal and may depend on the algorithms used; for example an Adaptive Cruise Control system (ACC: tactical level) might determine a target speed as reference for a simple classical feedback controller, while in collision avoidance the tactical level system may define a set of motion boundaries for a Model Predictive Control (MPC: control level) to use in the integrated control of steering and brakes. The separation is clearest when looking at the information processing demands; the tactical level must sense and interpret the driving environment, while 
the control level is required only to give best available performance based on objective information supplied. The upward arrow, from control to tactical (also from tactical to strategic) is included to indicate pathways for adaptation. Of course there may be other information flows, for example if a brake controller directly sends friction information to a traffic control centre, but Figure 2 is intended to show the major pathways. Note that from the "ABS perspective" mentioned above, most significant developments have emerged from the control level, albeit with significant intrusions into the tactical level.

The focus of this paper is to frame concepts and review progress and challenges in the area of vehicle and driving automation which have particular implications for the community of researchers and engineers in vehicle dynamics and control. This is also intended to move discussion away from an existing narrow and perhaps naive view that to replace the driver in a vehicle simply requires the removal all human elements from the three levels in Figure 2, achieving this via expanded sensor sets and information processing by artificial intelligence algorithms. It is born of the belief that what is already known about tyre mechanics, vehicle stability, direct yaw moment control, friction estimation, driveline control, brake system performance, driver modelling etc. etc. are important to the development of the new integrated technologies and will crucially influence their performance and safety.

This defines a convenient starting point for the main thrust of this review paper, which is organized as follows. In Section 2 we review some key developments that have led to the current generation of autonomous functions on road vehicles, with focus on those systems which first ventured into the tactical level of driving. Then in Section 3 we review another categorization of "levels" of driving automation, and based on this we consider the recent progress made at both the lower and higher levels. For intelligent vehicles to seriously enter the tactical driving domain requires high degree precision sensing of the environment; in Section 4 the issues of sensing and localization relative to maps - or directly relative to road boundaries or threats etc. - is considered. In Section 5 we review progress in establishing the safety of automated driving systems, noting that some raise safety concerns via possible unintended consequences, while others are specifically designed to protect drivers and others, e.g. via collision warning and prevention. Automation offer potential for precise motion control, and in Section 6 we review progress and opportunities for using optimization and optimal control techniques for benefits in energy, transport efficiency, individual time-saving (mainly at the largescale). Another design concept, away from optimal driving, is naturalistic driving aimed at creating automated driving systems that are 'less robotic' and perhaps more acceptable to vehicle occupants and other road users - Section 7. Then in Section 8 the focus is on the automation of limit handling, with applications for collision avoidance and other near-crash active safety systems. Finally, in Section 9 we focus on what is feasible and desirable for increased automation at all levels, including migrating towards full driving automation in the future. In particular we focus on which opportunities and challenges are most significant for researchers in vehicle dynamics and control.

\section{Evolution of the Intelligent Vehicle}

As described above, an initial electronic control evolution took place in the area of ABS and ESC for chassis systems; prior to this was the development of Electronic Engine Control (electronic fuel and mixture control, replacing the mechanical carburetor) which led to the further development of vehicle speed regulation in conventional cruise control. However, with no relevant environmental sensing in these systems, they all sit at the lower 
control level of driving of Figure 2. To contribute to the tactical driving the "Intelligent Vehicle" is directly aware of the driving environment and responds directly to it.

\subsection{Automated Speed Control}

Conventional Cruise Control (CCC) are popular features on cars, particular in countries where the roads are longer and straighter and destinations are farther apart, such as Australia and North America. CCC allows drivers to set a maximum speed at which they want to travel and the system controls the throttle and maintains the speed of the vehicles even on steep gradients. Despite the popularity of CCC there are relatively few studies that have examined the safety benefits/ disbenefits and acceptance by drivers [12], [13]. By adding a forward-pointing radar, a vehicle gains sufficient environmental information - range and range-rate of the preceding vehicle [14] - to adapt to traffic by reducing speed when there is a slower vehicle in front, otherwise tracking the driverselected set speed. This provided an early step into automating aspects of tactical driving in the form of adaptive cruise control systems (ACC). ACC was introduced in the market in 1995 by Mitsubishi in Japan [15] and rapidly gained acceptance. Today a growing list of car manufacturers offer ACC, including Cadillac, Daimler, Jaguar, Honda, BMW and Volvo; this is not an exhaustive list. For a more detailed description of ACC, including many references, see [16] and [17]. A number of studies have shown the trend of ACC driving to reduce tailgating as well as reducing workload for the driver. Early research was conducted at the University of Michigan and reported in [18] and similar findings were reported in [19]. On the other hand there is the potential for over-reliance on the automated ACC system, some authors have suggested potential pitfalls, for example due to misunderstanding of the ACC functionality leading to an increased collision risk [20], and also due to disengagement from the driving task leading to an increased response time [21],[13] as illustrated in Figure 3.
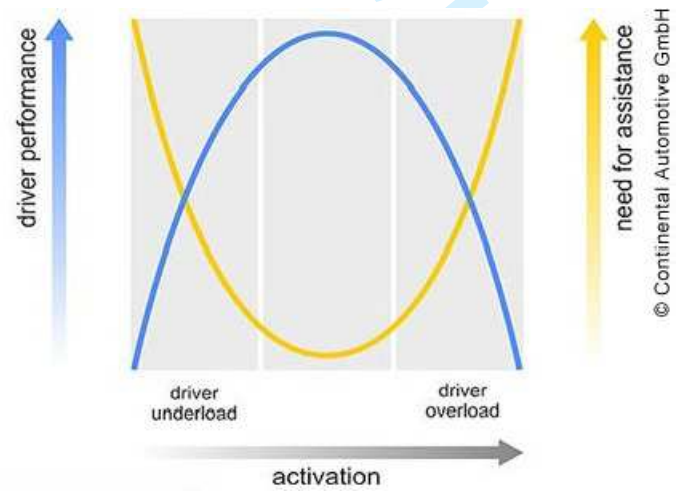

Figure 3. Driver performance and need for assistance depends on the activation of the driver (Picture: HAVEit project, Continental Automotive $\mathrm{GmbH}$ ).

While ACC is a comfort system, designed to assist the driver and reduce workload, it does directly relate to a form of conflict and crash i.e. forward collision. It is not directly designed to deal with such conflicts; if the car in front brakes suddenly and beyond the authority of the ACC, there is a warning issued and the driver is expected to take over speed control. Hence there exists a corresponding opportunity for accident prevention or mitigation in the form of Autonomous (or Automatic) Emergency Braking (AEB) ([22], $[23])$. Here the sensor and actuator requirements are essentially the same as for ACC, 
but the control algorithms are very different and control authority is increased: system activation occurs without any driver command, and high levels of deceleration result from that activation. Thus, as a result of the technical opportunity, collision avoidance and crash mitigation systems came onto the market as further developments of automatic speed control. In these systems, the car, or lorry, automatically applies its brakes to avoid a crash or reduce the severity of an anticipated impact. This is another example of existing actuators and sensors from ABS and ACC being co-opted for a new purpose, with the vehicles computer given authority to apply the brakes even when there is no command from the driver. The system's role is elevated from reducing the drivers workload to preventing crashes ([24]). In fact, it is part of the evolving new generation of autonomous safety functions in passenger cars and heavy vehicles.

\subsection{Automated and Shared Steering Control}

There is equal opportunity for automating directional control. Usually directional control comes from turning the steering wheel. Typical demonstrations of self-driving show the steering wheel moving by itself. As with speed automation, the key requirement is to sense the cars motion relative to the road, and/or relative to other road users, and then to apply path corrections to reduce or remove errors between the actual and intended paths. Since it is usual - though not universal - to have a direct mechanical connection between the steering wheel and the direction of the front wheels, there is a design challenge to best assist the driver with steering. One option is to use an Electric Power Assistance System (EPAS) to adjust the steering. This is already common in the power steering of more recent vehicles, where an electric motor replaces the hydraulics. When used in a more interactive or intelligent manner, the car will appear to want to steer of its own accord. Clearly, with hands on the wheel, the driver will perceive the car as having its own intention. Provided drivers are sympathetic to that intention, they will likely allow the steering action. EPAS is already used in self-parking systems where the steering action is fully automated - drivers remove their hands and simply control brake and accelerator during parallel parking. On the other hand, should unexpected steering torque be experienced during regular driving, this could disturb the driver and cause them to resist, which could have dangerous consequences.

There are other options for the car to control its direction, with different implications for the interaction with the driver. For example, with active front steer, an electric motor controls an offset between the steering wheel and the front road wheels [25]. This corrects the steering on the road without creating a corresponding movement of the steering wheel.

In another approach to automated steering, steer-by-wire removes the mechanical connection between the steering wheel and road wheels and so offers further scope to modify the interaction with the driver [26] (Figure 4). This type of electronic control can be used to steer the rear wheels as well as the front, giving more freedom to designers. Yet another option for directional control is to create a left/right bias in the brakes or driveline [27], [28], [29]; for example, by driving the right wheels harder than the left, or braking the left wheels more than the right, the net effect is to turn the vehicle to the left. All the approaches mentioned have their merits and implications in terms of cost, complexity, effect on the vehicles motion and, perhaps most importantly, their synergy with the human driver.

Whatever the mechanism, it is clear that sensors and actuators can provide an automated steering function. As with speed control, the system can function in a self-steering mode only if there is a reference direction/path to follow. This direction can be determined either by the vehicle alone, acting in autonomous mode, or, working in shared- 


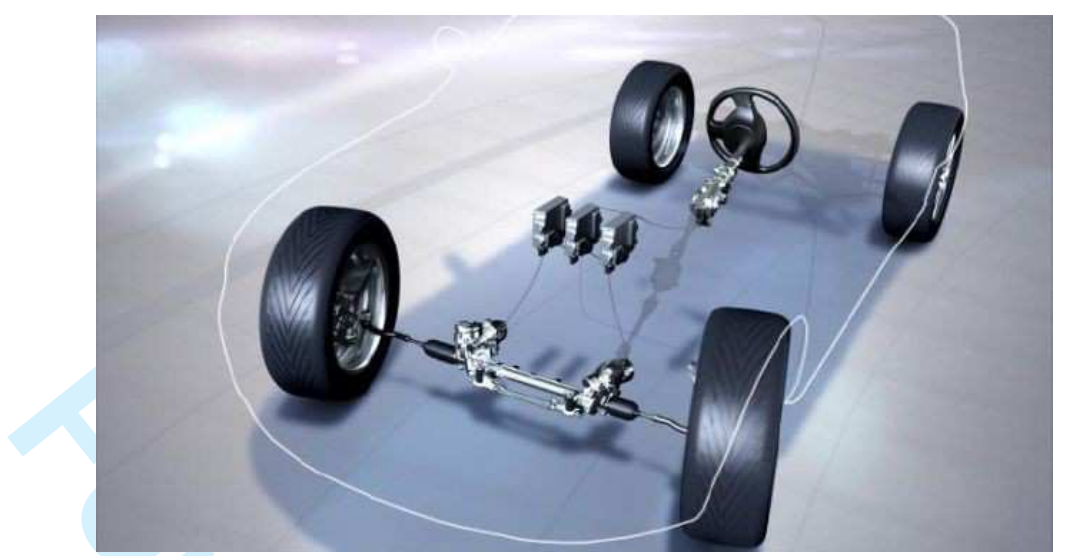

Figure 4. Steer-by-wire system with redundant electronic control units, a backup clutch system that would connect the steering wheel to the tires mechanically if a power failure occur (Picture: Nissan Motors).

control mode, as an adjustment to what the driver does with the steering wheel. In either case, the vehicle needs to know its position and motion relative to some map of the road and potentially to obstacles, including other vehicles.

In one form of shared steering control, lane-keeping assist or lane-keeping aid (LKA), now available in cars such as the Volvo V60, a camera determines the car's position relative to the lane markings. The electronic steering function then keeps the car in the lane [30]. The steering control is gentle enough to allow the driver to override the system for lane changing or to avoid an obstacle, in much the same way that ACC will defer to the driver for braking or acceleration [31]. In both cases, the responsibility for safety and legal liability stays with the driver.

LKA may also monitor whether the driver is holding the steering wheel. If the drivers remove their hands for more than a short time, the system will give a warning and then disconnect itself. Automated steering can also be a safety enhancement. For example, side-pointing radar can detect another vehicle in the blind spot in an adjacent lane so that the system will resist the drivers steering action if the driver starts to make an unsafe lane change. It is currently difficult to predict the actual safety benefits of such systems [32], even using so-called field operational tests.

\subsection{Semi-Autonomous Driving Systems}

We have seen how the intelligent vehicle with ACC and LKA has emerged from the initial electronic evolution of chassis and powertrain systems. A combination of ACC and LKA could potentially give us an autonomous driving system. However, both systems have limited capabilities: neither is designed to take full authority from the driver. It follows that we still require the driver to supervise: feet off the pedals, hands off the wheel, but eyes firmly on the road. And, importantly, the driver/supervisor should be mentally alert and primed and ready to take control at any instant. Several companies are developing such semi-autonomous systems with the motivation of further reducing the driver's workload. However, the shortfalls of boredom and distraction during a lowlevel supervision task are well known which is why lifeguards at swimming pools are rotated after a short time of supervision.

When the driver has to remain vigilant one doubts there is much genuine workload reduction. On the positive side, the driver can relax their arms and let the steering wheel 
move by itself. On the negative side, there is the new task of supervising and monitoring the automated system, and deciding if and when to take back control. The gain is a reduced physical demand, while the new burden is largely cognitive. Crucially, the semiautonomous vehicle does not alleviate the normal task of anticipating traffic hazards. Added to this are the dangers of overreliance on the system and being distracted by other tasks, potentially leading to new crash risks. This acts as an incentive for creating forever more ingenious systems which reduce crash risk, while at the same time allowing eyes off the road driving.

\section{Levels of Automation}

The large interest in on-road vehicle automation from a wide range of academic, industry and governmental communities has raised the need for a common terminology with a taxonomy and definitions. The new standard J3016 from SAE International simplify communication and facilitate collaboration within technical and policy domains [33]. According to the standard, the levels of driving automation can be divided into Conditional, High and Full Automation, see Figure 5. The standard does not provide complete definitions applicable to lower levels of automation (No Automation, Assisted, or Partial Automation). Active safety and driver assistance system that intervene to avoid and/or mitigate an emergency situation and then immediately disengage are also not included for the various levels of automation.

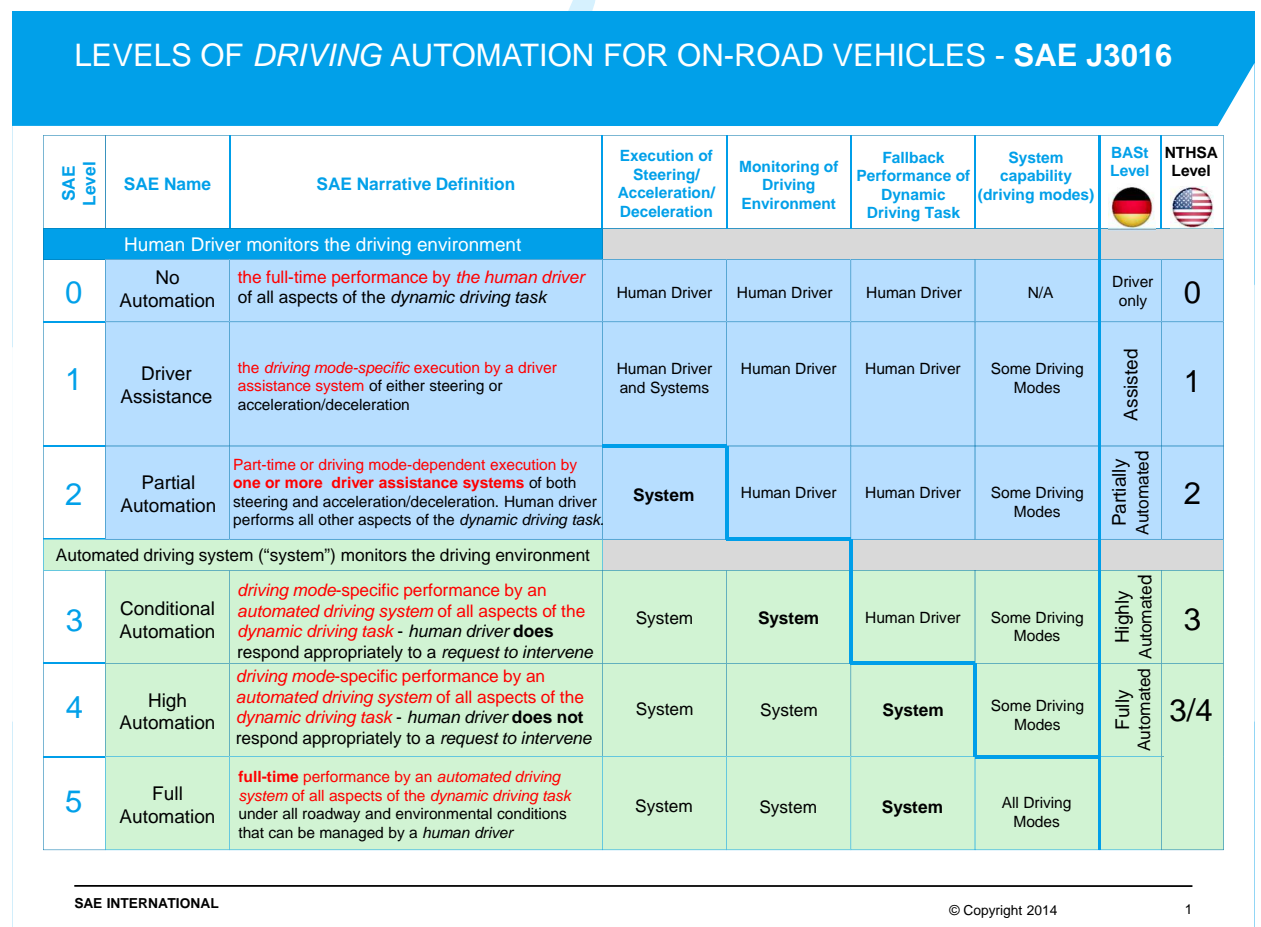

Figure 5. Summary of levels of driving automation for on-road vehicles (after SAE International [33]). 
Considering the level of driving, the human driver continues to perform tactical observation and planning for lower levels of automation. For example, using ACC, the driver is assisted with automated speed control but with steering still under the control of the driver, he or she needs to monitor all aspects of traffic dynamics and road geometry, including being alert to hazards. Going from Partial to Conditional Automation a transition time of several seconds is assumed, during which control can be handed back to the human driver; therefore aspects of tactical driving are shifted from the driver to the automated driving systems. For High Automation the capability is assumed for the automated systems to transition to a minimal risk condition in all situations, so the driver is no longer involved in tactical driving for modes that are automated in this way.

For Conditional Automation (Level 3) the system is expected to perform all modespecific aspects of the dynamic driving task with the expectation that the human driver will respond appropriately to a request to intervene. In cases where immediate release of the driving by the automated driving system could compromise vehicle performance, the system would be expected to delay its release - i.e. gradually relinquish control to ensure a smooth transition. For example, if the vehicle is engaged in a tight turning manoeuvre, the automated driving system should not release steering control instantaneously, but instead do so gradually as the driver indicates through steering input that he or she is fully re-engaged [33]. Needless to say, this puts new and increased demands on the driver interface, for example via active torque feedback in the steering system; potentially it also implies the need for sufficient machine intelligence to manage safety critical situations for several seconds at a time.

As pointed out earlier, it cannot be assumed that reducing the human driver's involvement in the dynamic driving tasks will necessarily reduce workload. This depends on the level of confidence the driver gives the system, and whether is always works seamlessly and reliably. The system also needs confirmation that the driver is sufficiently alert to recover control, so there may be a workload overhead for the driver in supervising, and an extra demand on the system to monitor and possibly alert the driver to stay engaged [33].

In the following we focus mainly on demands and current progress around Highly Automated Driving. However, we also briefly consider how conventional aspects of vehicle control, even at Level 0, make an effective contribution to the state of the art for driving automation.

\subsection{Conventional Automation}

A review of the conventional control problems of road vehicles - in particular the optimal control of vehicles and systems - was carried out by Sharp and Peng [34]. The major areas were deemed to be as follows, although the authors did state it may not be complete: (a) active and semi-active suspensions; (b) worst-case manoeuvring; (c) minimum time manoeuvring; (d) driver control modeling; (e) state estimation; (f) obstacle and collision avoidance; (g) integrated motion control; (h) spark-ignition engine control and (i) transmission clutch torque control.

Even with the present focus on automated driving, it should be clear that all are relevant to some extent. Of least immediate relevance are suspension control (a), powertrain control (h), (i) and perhaps (b) - worst case manoeuvring aimed at finding worst cases for vehicle systems to be tested under; for automated driving such cases are the more complex driving scenarios, for which a simple "worst case" analysis may not be credible. Prior research into minimum time manoeuvring (lap time optimization for race cars) is perhaps relevant, as it concerns "optimal driving" under friction limited conditions, 
so the research methods may be applicable in part in areas such as collision avoidance. Without going deeper into the specific areas, the conclusion is that most of 'basic' control methods of vehicle system dynamics are relevant to the automation of driving systems - we should beware of creating artificial separation between 'modern' and 'traditional' problems of automation and control.

We should also be wary of somewhat artificial boundaries between tactical and control layers: improving the precision of even basic manual control functions can have an effect on the way drivers interact with vehicles. A relevant example here is the EPAS system, which has been available in cars for some time. This has recently emerged as ElectroHydraulic Power Assisted Steering(EHPAS) for heavy trucks [35]. EPAS and EHPAS can not only improve the precision of vehicle control and the comfort for the driver, but also meet the higher requirements put on the steering system from Partial Automation and Conditional Automation. This comes from the substantial improvements in force feedback provided to the driver ([36], [37]). Basically, with the same philosophy, the improved precision of braking (electro-hydraulic, electro-mechanical, full electric control of torque), traction and torque vectoring systems [38] can also contribute in enabling control level functions to migrate vehicle capabilities up to the tactical level. Or, in other terms, without adequate precision at the control level, such migration towards 'dynamic intelligence' on the vehicle may be difficult or impossible to achieve.

There is another, perhaps unexpected, link between control-level technology and high level automation. For High and Full Automation the need for mechanical connections between driver and actuators (e.g. steering) becomes less relevant; in Level 4 (high) automation, there is no expectation for the driver to take over control in an emergency, so why impose the constraint of retaining mechanical connections? This immediately opens up the scope for a full by-wire automated car control similar to the by-wire approach of modern aircraft. As for an aircraft, the by-wire technology is designed to handle the major control functions using electronics only. The research and development challenge is to make use of system-level redundancy to prevent serious control degradation in the event of any single point failure. Certainly there are considerable safety implications associated with by-wire controls [39], but these are somewhat dwarfed by the other safety and reliability challenges introduced by Level 4 driving automation.

\subsection{Highly Automated Driving}

As mentioned, currently there are several commercial and academic groups working to develop automated vehicle platforms towards Level 4 capabilities. There have also been a number of larger-scale demonstration systems, plus some niche commercial applications that are operational and in service. Such systems, which expand beyond a single vehicle concept, make an attempt to reduce the demands of tactical driving and in many cases do this by simplifying the driving environment and hence reducing demands on the intelligent vehicles. In August 1997, a large research and development project ended with a live proof-of-concept demonstration of a prototype automated highway system, with 20 self-driving cars operating over a four-day trial period on dedicated lanes of Interstate 15 in San Diego, California [40],[1]. With speed and directional control both automated, the system used permanent magnets embedded in the road acting as a virtual guide-rail. Of course, manually driven vehicles were excluded from these dedicated lanes, and combined with the magnets we immediately see that the everyday demands of tactical driving are mostly removed.

Because platoons of vehicles are to be moving together, there is the possibility of an instability occurring; when a lead vehicle accelerates for example, the following vehicles 
may need to accelerate progressively harder to catch up, leading to a slinky effect or string instability. While this is not a truly unstable motion for a platoon of finite fixed length, it presents the possibility of unbounded response as the platoon length increases. This can be unnerving, and - for sufficiently long platoons - could lead to collisions whenever the lead vehicle brakes suddenly. Interestingly, the 1997 demonstration showed that automated vehicles can control platooning more precisely than human drivers; this was possible due to feedforward control from the lead vehicle, which used a wireless link to give prior warning of speed changes to all other members of the platoon. In this way, vehicle-to-vehicle (V2V) communication was used to improve performance of the automated system. With close spacing achieved at highway speeds, the demonstration showed potential advantages for increased traffic flow via reduced aerodynamic drag among the following vehicles.

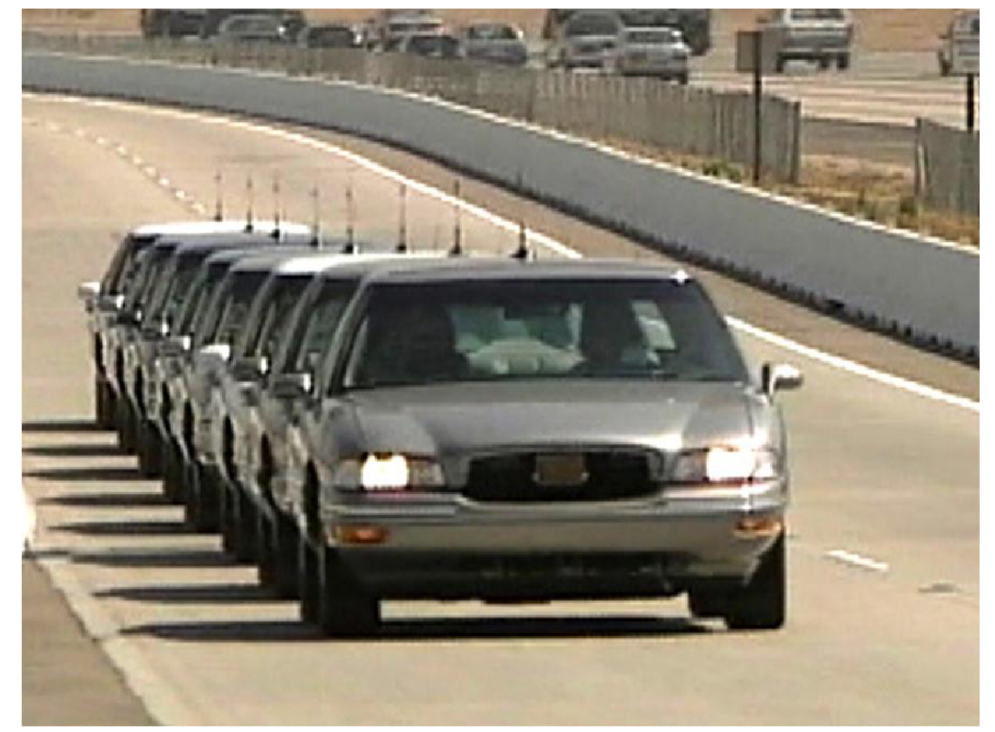

Figure 6. Live proof-of-concept demonstration of a prototype automated highway system, with self-driving cars operating over a four-day trial period on dedicated lanes of Interstate 15 in San Diego, California 1997 (Photo: California PATH Program).

Again, in a recent EU project, Safe Road Trains for the Environment (SARTRE) [41], led by Ricardo UK, a platoon approach was used. In this system, a heavy truck with a human driver at the wheel acts as platoon leader was followed by suitably equipped and highly automated passenger vehicles. With highway speeds and close spacing, this mother-duck/baby ducks scenario allowed drivers of following vehicles to enjoy eyes-offthe-road time. The approach is broadly feasible on existing highways as it simplifies the driving environment for each baby duck, mainly at the level of tactical driving. SARTRE is an interesting example of using a novel approach to deal with the complexity of the normal traffic environment.

A third and very different example of controlling the driving environment goes beyond the demonstrations of concept and further highlights the role of ensuring safety via a highly controlled infrastructure. Personal Rapid Transit (PRT), or podcar, is a public transport system featuring small automated vehicles operating on a network of specially built guide ways. Since 2011, PRT pods have ferried passengers between Heathrow Airports Terminal 5 and a nearby parking area [42]. With speeds up to 25 mph, self-driving electric cars run on dedicated tracks, with boundary fences, concrete barriers that sep- 


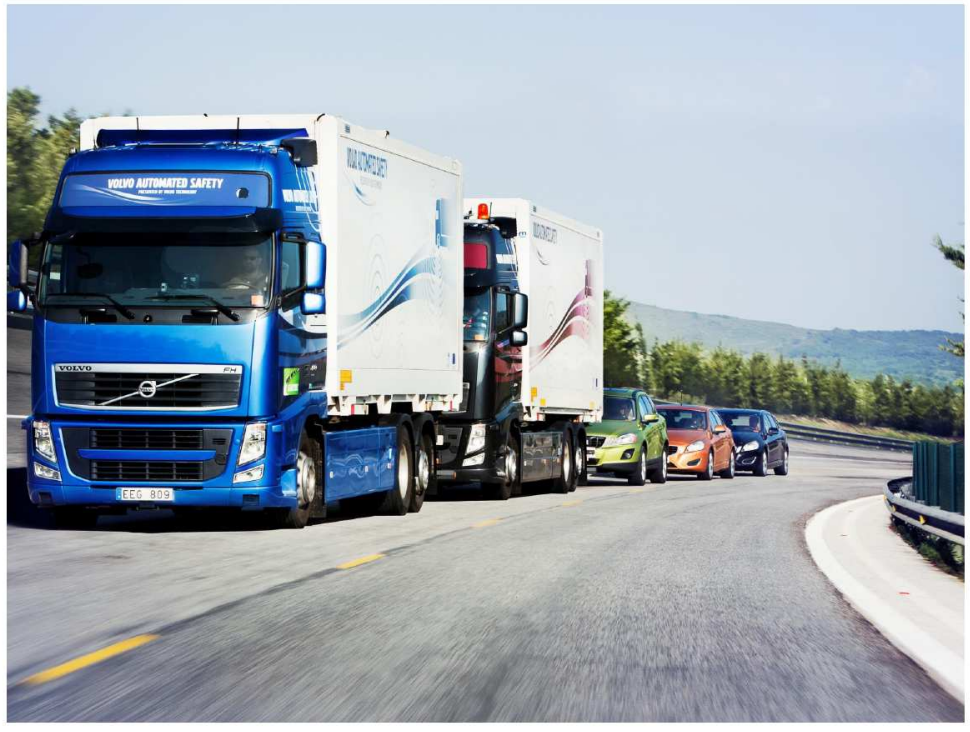

Figure 7. Safe Road Trains for the Environment (SARTRE) (Photo: The SARTRE Project).

arate adjacent lanes, CCTV and other real-time monitoring, plus the safety net of an independent collision avoidance system within the pods. The system, akin to a flexible rail system, is a long way from the freedom of general purpose self-driving cars, but it demonstrates real value to customers and is already economically viable. PRT systems require dedicated infrastructure which is both costly and challenging [43]. A different pod system is planned to ferry passengers between the rail station in Milton Keynes and nearby shopping centres [44]. Fully autonomous pods will be trialed in Milton Keynes in 2015 by the UK Transport Systems Catapult. Ultimately ferrying passengers between the railway station and nearby shopping centres, the pods will run at up to $24 \mathrm{kph}$ and be tested on a pavement route agreed with Milton Keynes Council to see how they interact with the public. It is also worth mentioning that a number of automatically guided bus systems have been deployed, such as the Phileas system in the Netherlands [45]; Phileas uses magnets in the road to refine the position of the bus, and there is also a human driver to supervise and intervene where necessary (Level 2 according to Figure 5).

Whether in platoons, niche commercial rail-like systems, or at the level of an individual highly-automated vehicle, there is a need for a consistent control architecture. A simplified architecture for a 'typical' autonomous vehicle guidance system is shown in Figure 9. The guidance system is mainly composed of four modules: a trajectory/mode generator, a trajectory/mode replanning, a low-level control system, and finally the vehicle and the real-time environmental model. In this approach an external control reference is used alongside a path-following algorithm, splitting the motion control into two parts. This is a common approach but it is not without its possible drawbacks - see in Section 8 . In any case, the trajectory generator and trajectory/mode replanning roughly equates to the tactical driving elements of Figure 2, while the low-level control block is obviously equivalent to the control level of Figure 2. For integrated systems of the type mentioned above there are additional layers required for the coordination and integration. And, as with the rail-like systems mentioned above, there is a further need for one or more layers of collision protection. Of course, the systems for both automatic driving and collision protection have an absolute need to directly sense and interpret the environment, and the collision protection system requires a threat evaluation function. We now consider 


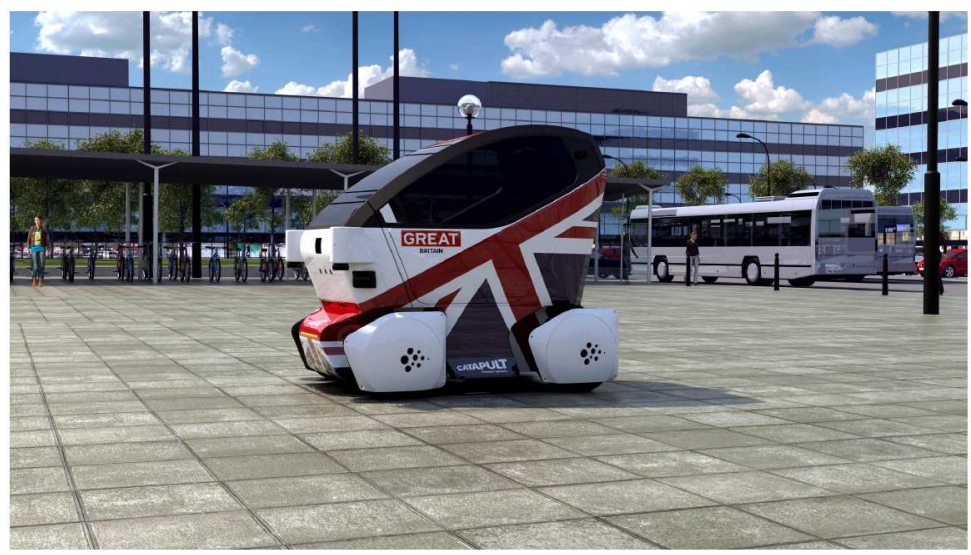

Figure 8. Milton Keynes Pods on pavement (Picture: The Transport Systems Catapult UK).

environmental sensors and their use.

\section{Environment Sensing and Motion Tracking}

At the most basic level an intelligent vehicle with automated driving capabilities needs to know where it is in the world, using some local coordinate system. It also needs to know the geometry of stationary features such as road boundaries and intersections, i.e. a local map. The map may be ad-hoc - created in real-time from sensors - as for example when an LKA system uses a suitably calibrated forward pointing camera to determine the locations of lane boundaries relative to the vehicle. Or a stored map may be used. In this case the precision of both the map and the vehicle location relative to the map are critical for motion planning and control. Currently there is a lack of publicly available maps of sufficient quality for direct use by automated driving systems, perhaps since

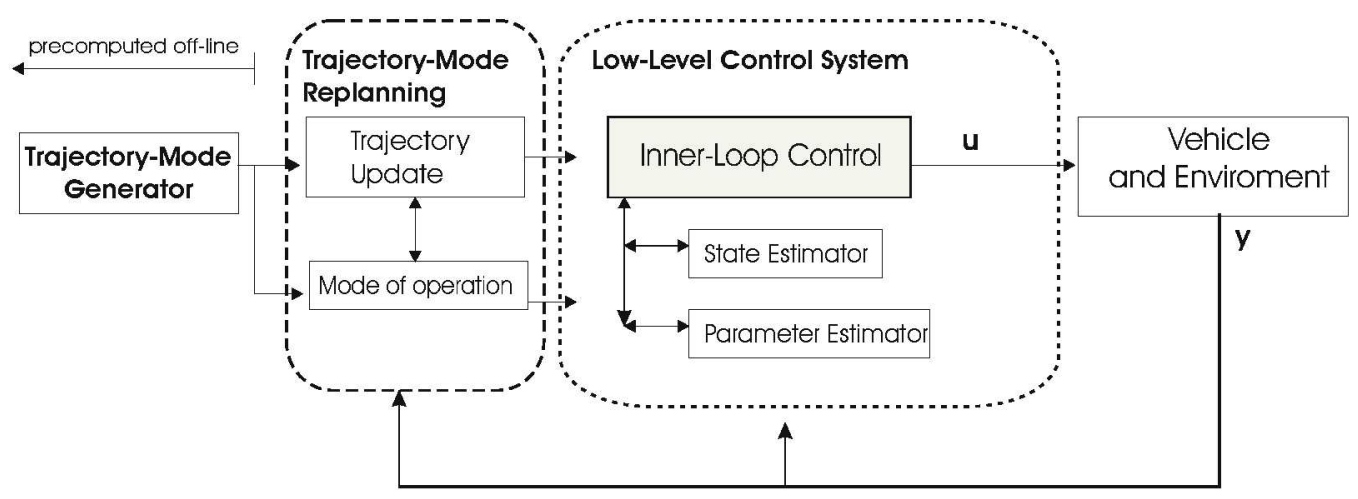

Figure 9. Simplified architecture for fully autonomous vehicle guidance system. (from Falcone [46]). 
the requirement for intelligent vehicle control is relatively new. This fact may have an important influence on the development of automated driving systems.

\subsection{Mapping and Localisation}

\subsubsection{Ad-hoc Mapping}

As a simple example, consider the position and mapping requirements of ACC. In the 'world of ACC', the ego vehicle drives in a single lane with limited curvature; the relevant elements of its environment are the lane markings and the vehicle in front (which may or may not be within range of the forward radar). Ego vehicle speed is estimated from the driveline, range and range-rate are deduced from a forward pointing radar, and road geometry is used to determine if a particular radar reflection comes from a vehicle in the same lane [47]; machine vision may also be used as part of this confirmation process. In this way a local map is created in an ad-hoc manner, and in particular it is not stored for later use. Although ACC (and LKA likewise) provide only a low level of driving automation, they share a number of the mapping and positioning requirements of other more highly automated systems. In particular, the control algorithms are based on a limited concept of the surroundings, in this case the world to the left or right of the current driving lane is conceptually outside the domain of ACC control and vehicles that 'cut in' from the left or right are recognized as new objects that appear into the ACC world. Of course the capability to pre-empt and accommodate cut-ins exists as a functional extension for ACC (hence expanding the relevant environment of the system).

Another example of ad-hoc local mapping comes from the MIT team that competed in the DARPA Urban Challenge [48]. In addition to the given 'route network definition file' (RNDF) and 'mission data file' (MDF) used for route planning, a large number of sensors were used, see Figure 10. These comprise odometry, inertial, GPS, LIDAR, radar and vision. Distance measurement from the vehicle (onboard odometry) can be from wheel or transmission speed; however this accumulates errors under even mild acceleration or braking and must be carefully calibrated for rolling radius, taking account of any changes in inflation pressure and vehicle loading. Two different GPS systems were used (one with an integrated inertial navigation system, INS), and three different ranging or imaging systems were also employed for tracking lanes and other vehicles as well as detecting drivable surfaces and obstacles. When the local map is connected to the world data from the RNDF file the result is an enhanced map, though again it appears that the map is not stored for subsequent missions; for the 'real world' of public highways it clearly makes sense to store and possibly share these kinds of data for reuse on future trips. It is worth noting that GPS was used as a reference to build local maps, but not directly for guidance, due to accuracy and availability concerns - see below.

From work by Paul Newman and his group in Oxford, it has been shown possible to use computer vision for building a map without any prior information [49]. Although this may have limited direct application to enhanced mapping of highways (accuracy levels are quoted as several metres extracted from a round trip of several kilometers) the power of the method is indeed worth considering for specialist mapping applications. The system uses stereo vision only and manages to 'glue together' successive locations of the vehicle into a comprehensive map. The system relies on static objects in the world being recognised in successive images, so that the change of pose (position and orientation) of the vehicle is found from the vision system and hence integrated into a time history of successive poses. And since the objects used for connecting the poses are an inherent part of the process, they can be fixed in the world. The system reduced drift by having 


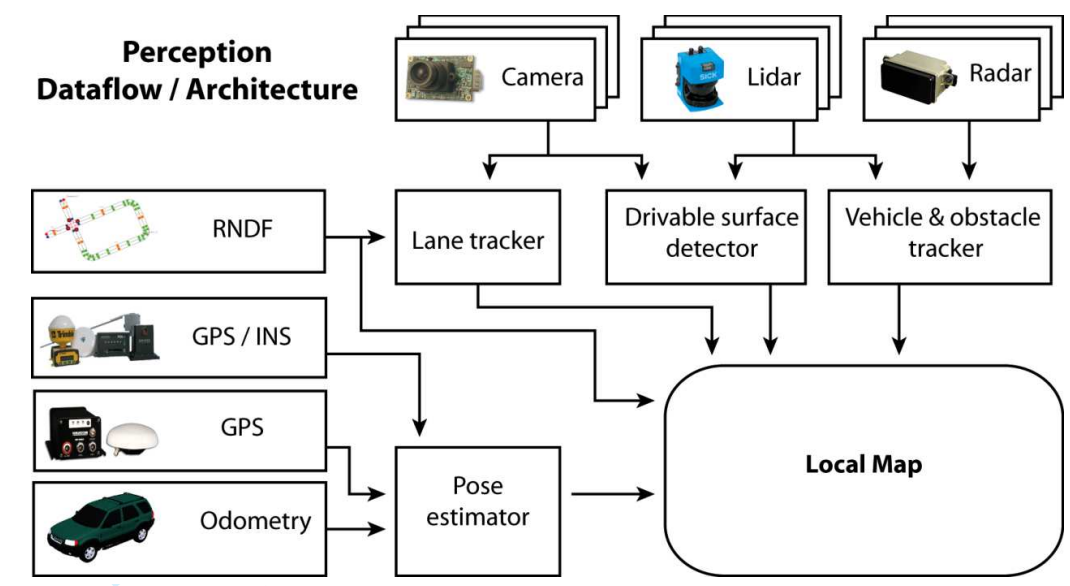

Figure 10. Hierarchical structure of the driving task including attention switching (from Leonard et.al. [48]).

the vehicle return to the start location and use loop closure as a constraint. It might be that this type of approach would be more accurate if it used a limited number of surveyed waypoints that could be recognized easily by the vision system. This would then represent a special type of map enhancement with the waypoints existing on a pre-defined low-resolution (but high accuracy) map.

Satellite positioning (GPS, or more generically and correctly Global Navigation Satellite Systems, GNSS) is ubiquitous but not always adequate for ad-hoc mapping nor for vehicle guidance. As mentioned, the MIT team from the DARPA Urban Challenge [48] did not rely on GPS for guidance, due to its relatively low accuracy and poor reliability of service, especially in an urban environment, where signals are blocked or suffer path reflections. While GPS is normally adequate for navigation systems, common experience shows that errors can easily exceed $100 \mathrm{~m}$. There are ways in which this baseline accuracy of GPS can be improved; for example Satellite-Based Augmentation Systems; Geostationary Navigation Overlay Service (EGNOS) or the Wide Area Augmentation System (WAAS) may be used to improve both the accuracy and integrity monitoring of GPS. However, even with such enhancement, absolute positioning accuracy is $\lesssim 3 \mathrm{~m}$ Assuming a sufficient number of satellites are visible, and in the absence of excessive multi-path errors (reflections from buildings etc.) RTK (real-time kinematic) differential corrections can be made to reduce errors to a few centimetres. But these systems are expensive and, while routinely used in professional mapping and surveying, they are not viable for commercial use on intelligent vehicles.

\subsubsection{Enhanced Digital Maps}

Humans don't need to learn the local geography in order to drive, and people can successfully drive in unfamiliar environments; but for real-world driving automation there are overwhelming advantages in using detailed stored digital maps to supplement what is available from published sources. Eventually such enhanced digital maps may be published, though a great deal of effort is required to establish this across the majority of the road network. Clearly it is wasteful and inefficient to build a new map every time the vehicle passes through a road segment, unless perhaps the level of detail is very low. Indeed, for the DARPA Grand Challenge, a critical success factor was the extent to which teams created enhanced maps prior to the actual drive [7], [50]. 
The requirement for map accuracy derives from the application, and in the case of automated driving functions the 'standard' requirement is lane-level accuracy. This is needed to support any plausible tactical capability, to determine which lane the ego vehicle is in (absolute error $\lesssim 1 \mathrm{~m}$ ) and hence plan for lane changes or take account of lane-specific traffic rules (e.g. right-turn only). This level of accuracy is also sufficient for other aspects of tactical driving, e.g. to plan deceleration on the approach to an intersection. Of course $1 \mathrm{~m}$ accuracy is not sufficient for the control level of driving; for example machine vision is still required to support lane-keeping systems, giving positional accuracy in the lane within a few centimeters.

Current publicly available road maps do not have this required accuracy; standard digital highway maps have an accuracy of only around 5 to 20 metres [51]. Furthermore they do not record the level of detail required - even to specify the number of lanes and whether the road has a central median is beyond the scope of existing digital maps. Hence some form of enhancement is needed via additional data gathering.

It is possible to automatically enhance existing low-resolution maps using data from dedicated test vehicles. An example is described in [52] using a survey vehicle and making use of publicly available maps from OpenStreetMap (OSM) [53]. At the core of this work is a commercial 'survey-grade' GPS system (Applanix POS-LV 220 inertial GPS navigation system) used to define the pose of the survey vehicle. The system incorporates two GPS receivers, inertial guidance and a rotary encoder for one of the non-driven road wheels. With additional sensors to capture road markings (camera and LIDAR) it was shown possible to produce map data with an error range $\pm 0.22 \mathrm{~m}$ and with mean absolute errors in the order of a few centimeters. This compares with a more standard survey system in [54] using the same type of GPS navigation; here - without any prior mapping - the stereo-vision system is capable of discerning features to within around 0.3 $\mathrm{m}$ (RMS) horizontally and $0.5 \mathrm{~m}$ vertically. Thus lane-level accuracy is available using only survey-grade GPS and optical/LIDAR imaging to create the required maps, without needing any fixed base station, and this remains true even when GPS coverage is limited [54].

A basic but important requirement for future use of stored enhanced maps is that they are maintained (reflecting updates to roads and lane layouts) and made available in a seamless way. Even outside of transportation applications, new mapping, data management and search techniques - in the form of Geographical Information Systems (GIS) has started to revolutionise the way maps are assembled [55].

Thus current maps are woefully inadequate for driving automation. Existing GIS data sets represent roads as a coarse series of nodes and shape points, which are connected by segments, and with no other salient features. In the future it should be expected that a great deal more data can be recorded and verified in available GIS data sets, including lane number, lane widths, median type and dimension, type and size of shoulder, quality of lane marking, presence and type of crash barrier, presence of rumble strip etc., all of which are relevant to driving automation.

\subsubsection{Localisation}

The position and orientation (pose) of an intelligent vehicle is needed in real-time (i.e. with limited and preferably constant time delay in processing). While the 'obvious' way to determine the position of an intelligent vehicle is via satellite navigation systems, we saw above that accuracy and availability are insufficient. If an accurate map exists, GPS corrections may be applied based on the assumption that the vehicle is on the road (map matching) though best-case positioning accuracy is no better than around $5 \mathrm{~m}$. Further 
improvement can be obtained from onboard odometry (distance measurements based on speed over ground or rotation of the road wheels) but lateral positioning relative to the road centre is not greatly improved. Another opportunity for real-time enhancement of positioning accuracy is to register fixed beacons or markers in the environment. These might be permanent magnets, visual markers readable by cameras, or they could be RFID (radio frequency identification) transponders. In [56] it is shown to be potentially feasible to achieve lane-level positioning using passive RFID tags in the road combined with electronic readers attached to the enabled vehicles. Position errors less than $1 \mathrm{~m}$ are predicted, though the study is limited to simulation.

It was mentioned previously [49] that stereo vision may be used to identify relative positions of fixed objects from frame to frame in an onboard visions system, hence identifying change of pose; but when there is considerable initial uncertainty in the reference object's location there is no guarantee that vehicle pose can be reliably estimated. It may be that a combination of lane markers, machine readable reference points, plus enhanced maps could provide the necessary reference for a vision system, but it seems clear that further research is needed on whether machine vision is sufficiently robust for the necessary localisation problem.

Another approach is to use DSRC (Dedicated Short-Range Communication, a secure form of wifi for highway applications) to enhance positioning. DSRC is expected to provide a variety of Vehicle to Infrastructure (V2I) and Vehicle to Vehicle (V2V) communication channels, as mentioned above for platooning. In [57] a V2I service is used to broadcast a differential correction to the DSRC-enabled vehicle ('connected vehicle'). In their experiments the additional service did indeed provide adequate enhancement but the reliance on GPS service remains troublesome. In [58] a rather novel approach is considered without GPS (other than to provide ground truth for evaluation). The system uses two fixed roadside DSRC base-stations within range of a DSRC-equipped vehicle (Figure 11). The base-stations broadcast their respective positions and the lane geometry as digital data. The wireless carrier frequencies differ based on doppler shift and vehicle location relative to the beacons. Using vehicle odometry to determine speed, the location of the vehicle can be determined. Success seems to depend on the distance between the base stations and their location relative to the vehicle, but the authors report accuracy that increases with vehicle speed and typically with RMS errors less than $0.5 \mathrm{~m}$ above very low speeds.

Much greater precision is possible using regularly spaced permanent magnets fixed in the centreline of the road [59]. In the work of the Partners for Advanced Transit and Highways (PATH) program at the University of California, vehicles sensed lateral position relative to these closely spaced magnetic markers, for which lateral offset directly 
fed into the steering control algorithm. Experiments showed that lateral tracking errors remained less than $10 \mathrm{~cm}$ under most conditions, and generally less than $15 \mathrm{~cm}$. Compared to other current approaches summarised above there are both advantages (high precision and availability of signal) and disadvantages (inflexible, does not support continuous localisation away from the chosen lane). Perhaps the clearest point of difference is that these markers were used directly for lateral control, rather than for lane-level positioning to support tactical aspects of driving automation. Current approaches rely much more heavily on GPS (when available) and vision systems to enhance the maps and also provide control level feedback (as in lane tracking).

It is worth mentioning that, just as GPS availability is a serious concern, so is the availability of other positioning systems. For a 'fleet' of embedded magnets, these would need to be maintained in a routine way if used on the highway; similarly, existing lane markings need to be repaired or replaced to support tracking by machine vision cameras. This is not always the case - for example, on certain road types and in adverse weather conditions, availability may be as low as $20 \%$ [32] though for highways it is closer to 90 $\%$, see Figure 13. Clearly, wet roads at night are a particular problem for machine vision systems.

\begin{tabular}{|l|l|r|r|r|r|}
\hline \multicolumn{2}{|c|}{} & \multicolumn{2}{l|}{ day } & \multicolumn{2}{l|}{ night } \\
\cline { 3 - 7 } \multicolumn{2}{|l|}{ clear } & \multicolumn{1}{l|}{ wet } & \multicolumn{1}{l|}{ clear } & \multicolumn{1}{l|}{ wet } \\
\hline Type A & rural & 90 & 90 & 90 & 20 \\
\hline & urban & 90 & 90 & 90 & 20 \\
\hline Type B & rural & 85 & 85 & 90 & na \\
\hline & urban & 70 & 70 & 65 & na \\
\hline Type C & rural & 50 & 50 & 50 & 20 \\
\hline & urban & 40 & 40 & 40 & 20 \\
\hline
\end{tabular}

Figure 12. Percentage availability of lane departure warning camera system used in South-East Michigan; Type A: Multi-lane divided, Type B: Multi-lane undivided, Type C: Two-lane undivided] (from Gordon et. al [32]).

Overall the tasks of mapping, map enhancement and localization of the ego vehicle relative to a local coordinate system remains a challenging area. Accurate information of this type is critical to all forms of automated driving control, though of course it is not sufficient. As noted, humans are capable of driving in the absence of such maps, particularly helped by familiarity with common patterns of road design. Of course accurate mapping and localisation is only sufficient for automated control if there are no other objects sharing the road surface along the planned path.

\subsection{Object Detection and Threat Assessment}

Sensor types for object detection and collision avoidance overlap with those used for guidance in driving automation. Additional types include short-range proximity radar and ultrasonic sensors, also for short range proximity detection. Unlike vision, LIDAR and scanning ACC radar, these proximity sensors have no way to recognize or classify objects, other than by context (e.g. because driver has switched on a parking aid, reflections indicate barriers or other vehicles etc.).

Autonomous systems for collision protection are being developed irrespective of any wider agenda to develop highly automated vehicles. An example is the Volvo City Safety 
[60]; the system monitors threat vehicles ahead via a laser sensor and brakes without driver intervention when an imminent crash situation is detected. This particular system operates specifically at short range and low speeds, and is from the general class of Automatic Emergency Braking (AEB) systems - Section 2.1.

There are clear overlapping demands between vehicle guidance and obstacle detection in the longitudinal direction. For normal driving, ACC radar or LIDAR detect vehicles in front, with the same or similar requirement as for AEB [22]. In both cases there is a need for a threat assessment (and intervention) algorithm, based on speeds and range, but the details will be different, particularly for the threshold levels to trigger a response. Thresholds for AEB activation are based on metrics similar to Time To Collision (TTC) or Last Time To Brake (LTTB) [61]. TTC uses range $R$ and range-rate $\dot{R}$, and is defined as the time to collision assuming constant speed of both vehicles, i.e. $T T C=R / \dot{R}$. LTTB uses more detailed information, including estimated surface friction, but in either case there will be uncertainty over any deceleration of the lead vehicle and AEB may not be expected to always prevent collision; but in all cases the relative speed at impact will be reduced by the intervention.

More capable collision protection systems require more sophisticated sensing and sensor interpretation, including multi-sensor fusion algorithms. For example the Volvo "Collision Warning with Full Auto Brake and Pedestrian Detection" (CWAB-PD) uses radar for increased range and monovision cameras for validating the type of object [62], [61] and hence can deal with a wider range of forward collision situations.

Threat assessment from the lateral direction is more specific to collision protection. A number of 'blind spot warning' systems have been on the market for some time; when there is another vehicle nearby in the adjacent lane a warning light illuminates to alert the driver that a lane change would not be safe. Alternatively a haptic signal may be used via the steering wheel [63]. These systems are activated by radar or cameras, which may also be used to trigger active steering resistance or path correction in case of an unsafe lane departure.

There are various other collision prevention systems for specific crash types, for example warning and intervention to avoid hitting a pedestrian when reversing a vehicle. In [64] a number of these systems, all using ultrasonic sensors, were evaluated. In some cases their effectiveness is questioned, mainly due to short range limitations, and especially those using sensors developed as parking aid systems; these detection systems sacrificed detection distance and height in order to suppress false or nuisance alarms. Again, while

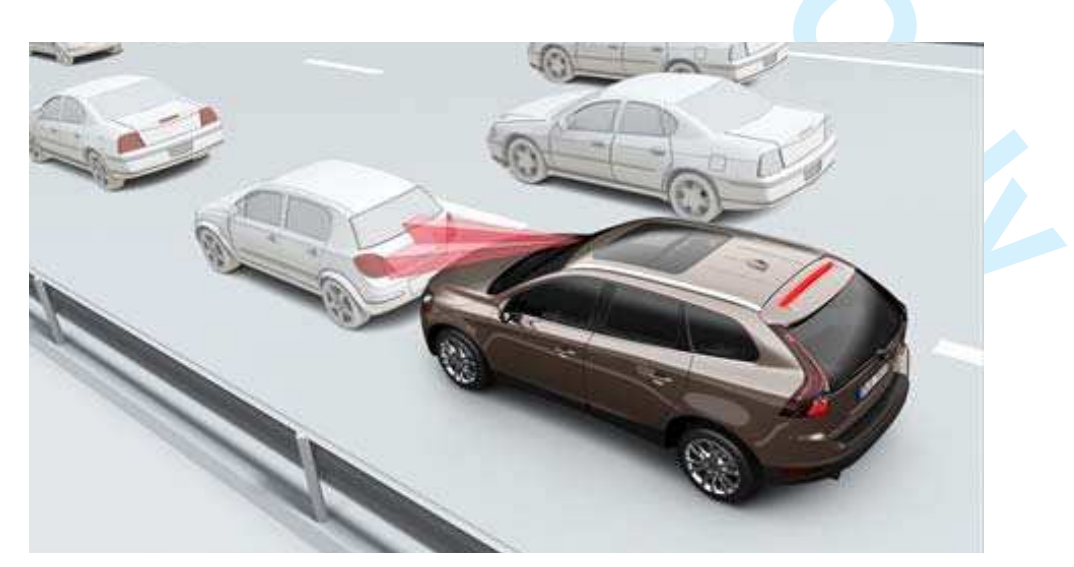

Figure 13. Automatic braking to avoid low-speed frontal crashes (Volvo City Safety system - from Distner et. al $[60])$. 
guidance and protection involve similar functionalities, their precise requirements are different and the systems should normally be developed independently.

Collision protection is often based on assumptions of deterministic intent of the threat object (e.g. constant speed or deceleration of a lead vehicle). However this is not necessarily reasonable or useful in the area of preventing car-to-vulnerable road user (VRU) accidents. In [65] there is an attempt to characterise, in a non-deterministic model, the likelihood of a pedestrian entering the road based on various factors: car speed and its distance to the point of a potential collision, pedestrian distance to the road, plus pedestrian speed and head orientation. Such a predictive algorithm could be worthwhile in reducing crash risk by alerting the driver or any AEB system. On the other hand it is not likely that a Level 4 automated vehicle would entirely rely on such a system, and yet a deterministic model would clearly be inadequate. This and similar crash modes may in the future define the environmental limits on the safe use of highly automated vehicles.

\section{Safety and the Role of the Driver}

Safety is perhaps the biggest single concern when considering the automation of driving. Each level of Figure 5 brings its own safety concerns, and in particular the question of 'what happens if something goes wrong'. It is hard to believe that a mother would send her child to school in a Level 5 self-driving car without severe trepidation, unless there were a trusted human being as part of a 'backup plan'. And the driver of a Level 4 platoon vehicle would surely be sensitive to whether the lead vehicle has a human driver (as in SARTRE) or not. It appears that the topic of risk and safety of highly automated vehicles is wide-open, given that predictive safety evaluation of road vehicles is at a very early stage, and there is little real-world experience to draw on.

At Level 0 there is of course a lot of experience, and it is easy to underestimate the safety performance of manually driven vehicles. According to US traffic statistics in 2012 [66], out of roughly three trillion vehicle miles travelled, there were a total of 5.6 million police-reported traffic accidents, 30,800 of them involving a fatality. This equates to roughly one crash per 500,000 miles and one fatal crash per 100 million miles. This compares to the currently quoted million-plus miles of accident-free (supervised) travel for Googles self-driving cars, where the development team selects roads and timing, intervenes when necessary, and presumably does so with high attention levels. For humans, tactical driving requires experience and attention. It is also the level that is most demanding for highly automated cars. It seems infeasible to replicate all aspects of human driving at the tactical level, at least not without artificially intelligent computers that are beyond anything currently proven. We might formulate a Turing driving test on this topic: if a self-driving car behaves in all traffic conditions such that other road users cannot tell any difference from a human driver, then we might assume that we have a safe and acceptable system. Of course the traffic conditions should include complex and dynamic scenarios.

Example complex scenarios are considered in [67] - see Figure 14. That work relates to highly-capable driver assistance systems: the driver support function (so-called 'codriver' in this case) needs to anticipate the intended manoeuvre and cannot do so unless the driver's goal is known (in this case either $\mathrm{a}-\mathrm{b}, \mathrm{a}-\mathrm{c}$, or $\mathrm{d}$ ). Each goal/manoeuvre has risks associated with it, and the co-driver must correctly anticipate in order to monitor traffic and reduce risk. This is an immensely difficult problem for which the support system could potentially increase risk due to unpredictable human-machine interactions.

This example illustrates that risk and safety prediction is highly challenging and not 


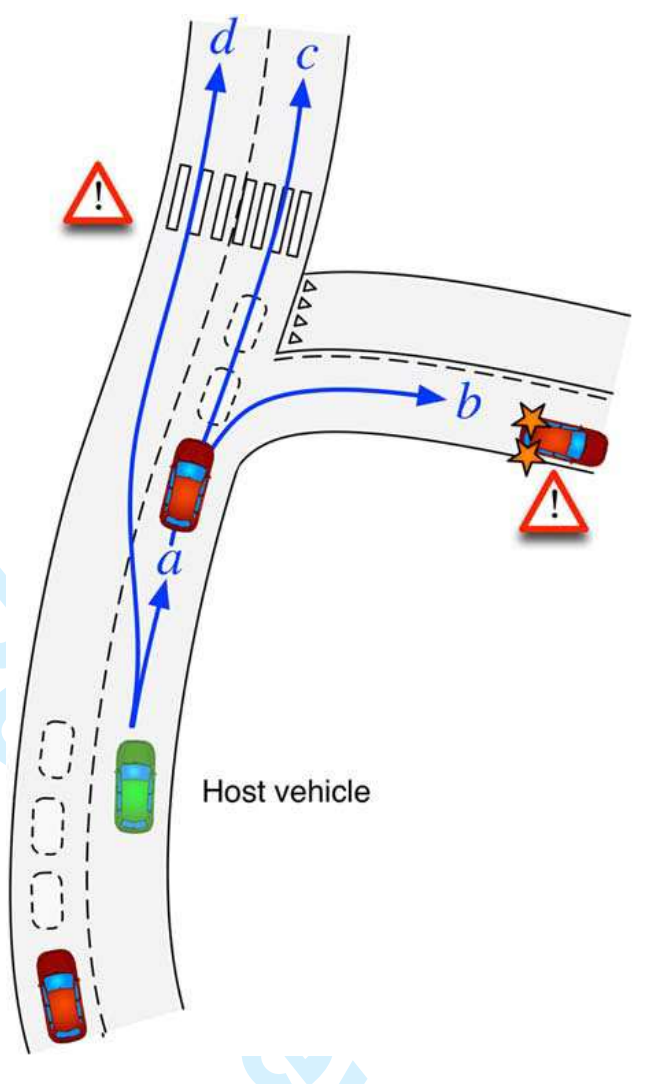

Figure 14. Complex highway scenario with multiple feasible outcomes (from Da et. al [67]).

just for highly automated vehicles. The HAVEit project [68] also considers the concept of driver support from a co-driver, including aspects of adaptation to risk and flexible levels of automation (see also Figure 3). But again the work does not provide predictive measures of crash risk.

For active safety systems there have been a number of studies assessing the effects on crash risk. When modalities are relatively simple it is possible to assess crash benefits by fusing a wide range of data sources, combining these via Monte-Carlo simulation - e.g. for Lane Departure Warning systems [32]. Real-world statistical analysis can be used for systems that operate very close to potential crashes. For AEB systems, it was reported in [69] that there is a significant reduction in crash numbers for the relevant 'striking rear end' crash type; in $50 \mathrm{kph}$ speed areas the reduction was $54-57 \%$, while over all speed limits the reduction was $35-41 \%$. The work was based on Swedish police-reported injury crashes between 2010 and 2014. A similar trend was reported in [70] for the Volvo City Safety system: the number of claims for property damage liability were $16 \%$ less for (equipped) S60s than for other (non-equipped) mid-size luxury cars.

Given that we cannot yet put human-like driving intelligence into automated driving systems, there are two complementary ways forward towards achieving high levels of automation. First, we can make best use of the available safety net of collision prevention systems, such as AEB. Over time, using crash data and improved scientific analysis, we can further evaluate and verify the safety benefits of such systems. These 'safety 
nets', developed to protect from errors by human drivers, can also provide protection during various levels of automated driving; thus driving safety benefits can be extended and validated in real-world studies. Secondly, we can simplify and regulate the driving environment to the point where crash risk can be evaluated in an objective way ahead of deployment.

\section{Optimal Driving Control}

Human driving may be far from optimal [71] - for example control time-delays are long compared with automated systems, and drivers' attention may be intermittently switched away from the driving task. Steering control typically has low bandwidth and maintaining lateral lane position is not necessarily a challenging control task, whether for a human or a machine. Under normal conditions, human driving is effective, and one might question whether automated driving systems should aim for optimality in any particular sense, even though a number of studies into synthetic driving systems (driver models) use optimal control methods at their core [34]. One might go further and seek to design automated speed and steering control systems that perform in naturalistic ways - sufficiently akin to human driving so as to be acceptable to vehicle occupants and to other road users. This is contrary to the idea of imposing a rigorous control regime positioning the vehicle as precisely in the lane centre as possible and maintaining precise headway - where such behavior may appear alien and distracting to other road users.

This concern suggests that research may be worthwhile into understanding how automated driving control should be capable of mimicking human behavior, at least in certain driving scenarios. But research is also needed to explore the benefits of precision and optimality when automating different aspects of the driving process - to improve safety during normal driving, to provide effective protection in safety-critical situations, and more generally to improve "softer" aspects of vehicle system dynamics, such as comfort and fuel economy, as well as reduced carbon emissions. Travel-time efficiency is also worth considering in this context.

The very concept of highly automated (self-driving) vehicles implies time saving benefits to drivers - being able to focus on other activities while automated systems perform all tactical driving and control functions on the vehicle. This is true, whether or not the stated aim is for safety improvement, fuel saving etc. Further, during normal driving scenarios, these systems have the potential to fuse real-time environmental information (e.g. traffic and topography) with data about the vehicle status (e.g. state of charge of the battery) to implement efficient control strategies that are beyond the interest and capability of human drivers.

One example of automated driving optimisation that requires such data fusion is the "pulse and glide" strategy for longitudinal control, aimed at minimising fuel consumption [72]. This expands on the simple idea that running an internal combustion engine close to its most efficient operating point will improve fuel economy. In the absence of a continuously variable transmission, a way to achieve this is to run the engine intermittently at a higher power in order to reach the efficient operating point. Even allowing for transient reductions in fuel economy, the pulse and glide strategy may be capable of producing around $20 \%$ improvement in fuel economy. But this is only possible if the intermittent acceleration and deceleration does not disturb traffic flow or cause other drivers to react. While the control concept may well suffer from obvious problems, particularly the discomfort of passengers [72], the fact that local vehicle optimal control can benefit from being sensitive to the interaction with the vehicle environment is interesting 
and probably quite general. The example can be described as having a 'weak' connection to the vehicle environment. Similar weak connections exist around traffic signal control and cooperative ACC (CACC - see below) which aim to smooth traffic flow, increase highway capacity and reduce transportation energy consumption with particular emphasis on vehicle communications technologies, either between vehicles - V2V, or between vehicles and infrastructure - V2I [73].

In other concepts, the traffic and physical infrastructure may require a much stronger connection.

\subsection{The Automated Highway}

Optimization of strategic driving in response to prevailing traffic conditions (real-time route planning) is a well-known problem in traffic network analysis [74] and since this problem is very much aimed at the upper level in Figure 2, there is little relevance to the optimal automation of driving processes, except for any resulting interactions between network level "control" and individual vehicle control. Referring again to Figure 2, until now we have considered the functions to be localized within the host vehicle, even though contributions from both human driver and automated driving systems may be involved. From the alternative perspective of "Automated Highway Systems" (AHS) or "Intelligent Transportation Systems" (ITS), there may be scope for the highway system to take over both tactical and strategic functions. Of course we always expect the low level control of vehicle systems to be handled locally.

Within infrastructure-centric control, common applications relate to traffic flow improvement via static infrastructure components, especially traffic signals [75], [76], [77]. Recent interest in highly automated vehicles has led naturally to traffic flow optimization studies that assume direct control of automated vehicles, and this requires a switch towards granting responsibility for tactical driving to a centralised transportation control system.

A conceptual example of such centralized control is the Integrated Roadway/Adaptive Cruise Control System (IRAC) [78] which assumes infrastructure-based controls for speed distributions along highway lanes as well as ramp-metering, i.e. traffic signal control of vehicles entering the highway. This study was based on traffic simulation, assuming the use of DSRC communication, and predicts improvements to traffic flow. More extreme example of centralized traffic control are presented in [79] where the authors propose a coordinated traffic control scenarios, where lane-changes are executed in the path of cooperating oncoming vehicles. Such scenarios are somewhat futuristic in terms of the level and scope of control that is required and raise questions over the effect of any singlepoint of failure - when one vehicle fails to cooperate there is near-certain collision. The optimization problem is limited to a local group of vehicles (around 40) and is applied at the level of discrete decision making. While the full idea is somewhat implausible (e.g. the number of collisions predicted are not vanishingly small) they do raise the potential for a new approach where a "smart infrastructure" provides an additional layer of tactical support, reducing the tactical driving demands on the individual vehicles.

\subsection{Platoon Control}

Travel efficiency was also the primary motivation behind the use of precisely controlled platoons as developed and demonstrated by the California PATH program described in Section 3.2 [40], [1]. Platoons are clusters of vehicles that synchronise their motion to 


\section{March 27, 2015 \\ SOA paper v11 \\ Vehicle System Dynamics}

form a closely-moving pack, acting as a single multi-vehicle 'object' on the highway. In the PATH project the assumption was that such a platoon will move on a dedicated lane within a tightly controlled AHS environment.

Several concepts from platoon dynamics, particularly the need to use wireless communication to suppress longitudinal disturbances (string instability), have led to research and development in the area of Cooperative ACC (CACC) where it is assumed that lateral control comes from the human driver but a self-organizing platoon emerges from local headway control (as for ACC) with the addition of cooperative elements which are mediated by wireless communication. One may think of CACC as a type of 'optimised' ACC system, even though optimal control methods are not necessarily used in the controller design. Experiments with this type of system took place during the 'Grand Cooperative Driving Challenge' (GCDC) where several vehicles were expected to cooperate in order to perform cooperative adaptive cruise control (CACC), [80]. While a number of practical and technical problems were revealed in this work, the basic feasibility of CACC is not in doubt. In [81] the authors summarise recent studies in CACC, noting that in most systems the control laws are based either on classical control theory or Model Predictive Control. Their own experiments made use of four Nissan vehicles which were equipped with LIDAR-based ACC plus a 5.9-GHz DSRC communication system; in their case the control algorithm was designed using standard classical control methods. Figure 15 shows the effect of braking from the lead vehicle, which decelerates at $0.1 \mathrm{~g}$. In the upper plot (standard ACC) there are clear response delays as the vehicles further behind successively respond to the braking event from the front vehicle. There is a visible amplification in the acceleration response of vehicles following further behind, and this is the characteristic symptom of 'string instability'; indeed the fourth car brakes at $0.3 \mathrm{~g}$. For CACC (lower plot) the acceleration is no longer amplified - there is improved gap control and the system appears to be string-stable (at least in and around the test conditions). While such results have been known for a long time [59], the results clearly demonstrate the practical advantage of using wireless communication when ad-hoc platooning occurs among several ACC vehicles.

Further research will be needed however to show how similar performance can be achieved using distributed control among heterogeneous groups of ACC vehicles. One possible approach to address this challenge is to adopt a control architecture that makes use of infrastructure components to provide a coordinating function. We discuss this further in Section 6.3.

Platoons have also been proposed for fuel and carbon saving. The German national project KONVOI focused from 2005 to 2009 on the topic of platooning, in which a series of heavy trucks follow a leader under fully automatic control, the small gaps intended to improve aerodynamics and reduce fuel use. V2V communication enables close spacing of vehicles, also leading to a reduction of occupied road area and an improvement in traffic flow. In fact the European projects PROMOTE CHAUFFEUR I and II were the first research projects in the EU on this topic, and they focused mainly on technical feasibility; the project KONVOI studied the impact (driver acceptance, traffic flow and environment) as well as the legal and economic implication of platoons [82]. The KONVOI system was the first platoon system worldwide, which was tested in real traffic. The trucks were equipped with a V2V and V2I communication system, a mono camera as well as LIDAR and radar sensors. On the basis of the real traffic drives it could be shown, that a safe operation of platoons is possible. Researchers claimed that the trucks on the test track achieved some fuel-consumption savings even when they were driving at the 10-m gap between trucks; however, there was no fuel-consumption savings in the tests on the public highway because the trucks had to vary their speeds to respond to traffic conditions and 

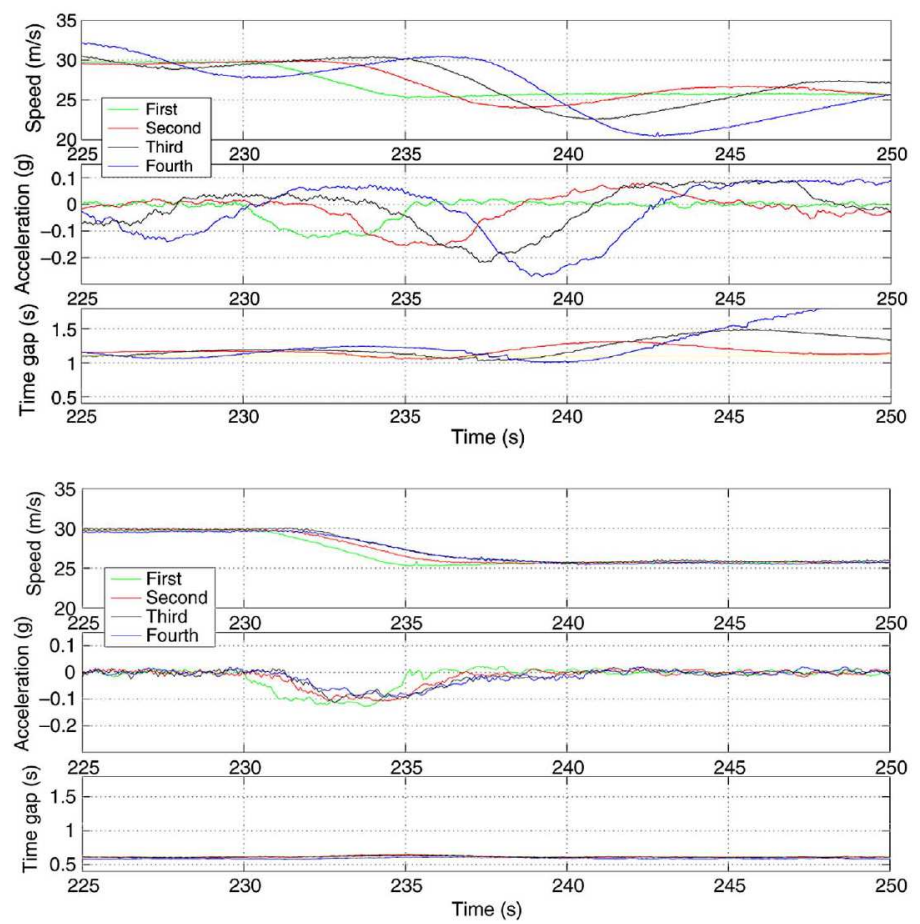

Figure 15. Real-world comparison of ACC (upper plot) and CACC (lower plot) responses due to braking of the lead vehicle. For CACC wireless communication enables a coordinated and string-stable response (from Milanes et. al [81]).

other vehicles on the road [82]. As previously mentioned, the European project SARTRE [41] explored vehicle platooning with a view to improving fuel economy; a manually driven truck is in the lead, while trucks or light vehicles following under automated control. The study included tests on public highways [83] and results showed fuel saving results of up to $15 \%$, see Figure 16.

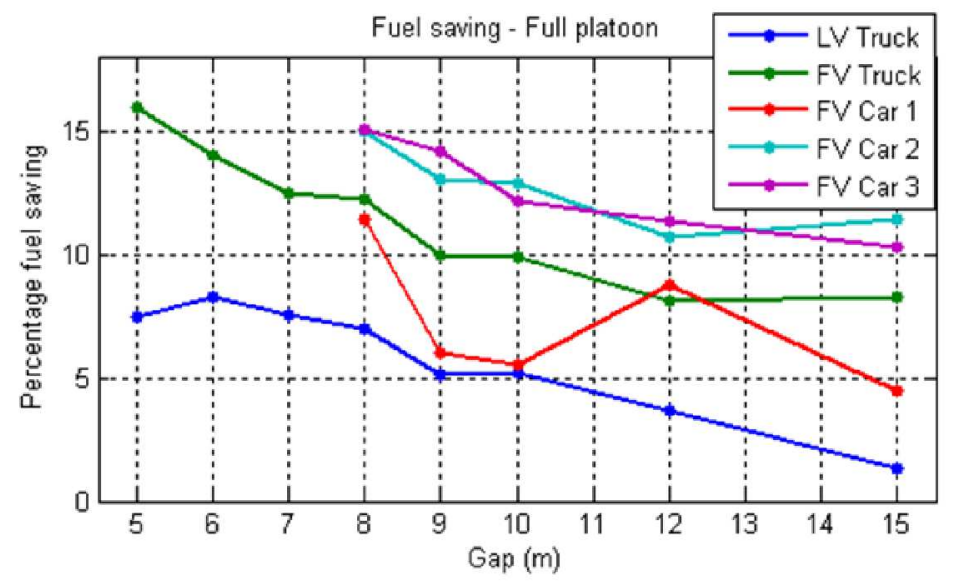

Figure 16. Fuel saving results from the SARTRE project: effect of inter-vehicle gap on fuel consumption reduction (from Chan [83]). 


\subsection{Tactical Support from Smart Infrastructure}

In the above it is seen to be important to reduce the demands on tactical driving to enable higher levels of vehicle driving automation. Information from maps and from real-time infrastructure communication systems can improve efficiency and safety. For example, road preview can be useful when controlling hybrid powertrains. In [84] using 5 to 15 seconds of preview control for traffic conditions can lead to useful fuel saving of 1-2\%, which is also similar to the topographic preview analysis of [85]. While these may be only modest savings, in city driving or at higher levels of automation, it can be expected that infrastructure support would have greater benefits on journey times and fuel efficiency.

In Figure 17 we propose the concept of tactical support from the infrastructure. One example for this is connectivity to traffic light controller which adjust their patterns to upstream traffic flows, and communicate this to DSRC equipped vehicles [86]. Another case relates to Level 3 driving where infrastructure surveillance (e.g. on motorways) could help guarantee greater time horizons for safe automated driving - to confirm that the traffic flow ahead is stable for some distance and that there are no intrusions by animals or other hazards. The tactical support function might operate as a kind of "ground traffic control' in communication with the automated driving system but not (as conceived here) with the human driver. According to this concept, a smart infrastructure provides tactical support to reduce the required intelligence for tactical driving, reducing the demands for on-board AI systems.

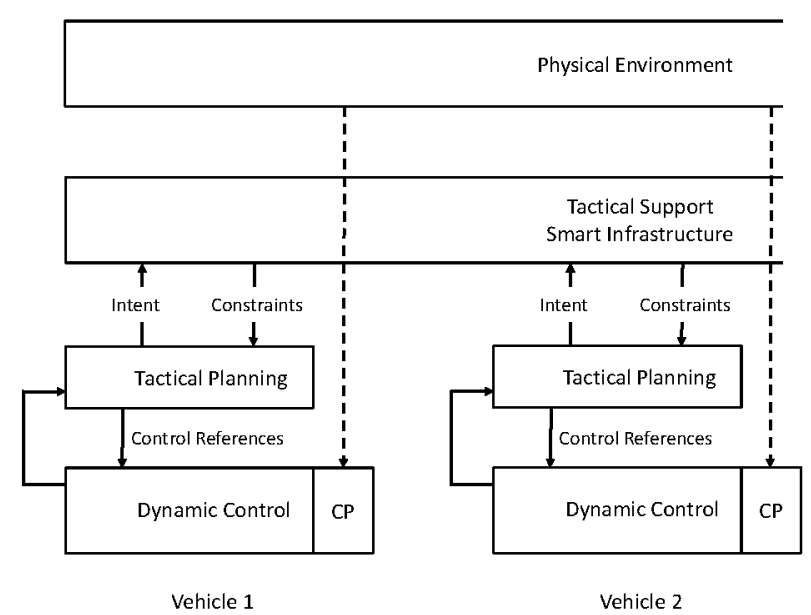

Figure 17. Tactical support from the infrastructure: an enhanced control architecture designed to support automated driving functions at all levels of automation. CP denotes Collision Protection, responding directly to the physical environment.

\subsection{Lateral control optimization}

For lane-keeping and lane changing the control demands are modest provided there is sufficient positioning accuracy within the lane(s) and that lane-level map positioning is achieved. As mentioned in Section 4, vision systems may be used to determine vehicle 
position relative to lane boundaries; in particular the previewed path error and local yaw angle error are needed; this is then sufficient to enable satisfactory lane tracking [87].

An alternative approach, avoiding the use of vision systems, comes from the California PATH program which adopted a magnetic road reference system (permanent magnetic markers - Section 4). Without preview the (linear) control scheme is prone to instability, since with the "look down" characteristics of a front-bumper mounted magnetometer, the sensor-to-response dynamics have poorly damped open-loop poles and zeros, which worsen at higher speeds - hence even a small feedback gain generates instability in the root-locus plot [1]; in more colloquial terms, it is not possible to drive at speed by looking at the road beneath the car. To overcome this problem it was found sufficient to include a second rear-bumper mounted magnetometer, and use the combination of sensor measurements (plus vehicle inertial sensors) to estimate path curvature. This allows the lateral controller to provide "virtual preview" and hence a more acceptable controller. In this approach, preview is only effective up to a few car-lengths due to sensor noise and variations in path curvature [1].

In [88] another enhancement is included, this time to use tracking of a vehicle ahead to supplement one of the magnetometers if the second one is lost. LIDAR was assumed in this work, but a vision system could also be used.

Optimal control methods can also be used for lane-keeping and simple manoeuvres such as lane-changing. In either case it is standard to assume a target path and then track towards that path. The control problem is not difficult, at least as long as the relevant vehicle positioning and map data (or equivalent) is available to compute previewed path errors. Again, one may treat the control problem as one of "synthetic driver modeling" and many optimal and operational methods are available [89], [71], [90].

The challenge of defining a reference path - as well as the motivation to avoid this step - is even greater in the case of collision avoidance relative to a moving vehicle [91]. In this work the scenario is relatively simple - a lane change - and the motion of other vehicles are presumed to be fully known. It does however include cases where the other vehicle is accelerating or moving in a curved path, so the approach is both general and informative. The optimization uses a cost function in the form of a weighted sum of squares $J=\frac{1}{2} \int_{0}^{t_{f}}\left(y^{2}+w \dot{\delta}^{2}\right) d t$ which penalizes control action (steering angle velocity $\dot{\delta})$ and deviation from the pre-defined reference path $y$. The authors use formal optimal control (Pontryagin Minimum Principle) and a linear bicycle model. While this provides a reference for comparison of more implementable control algorithms, the path definition is outside of the optimization and the use of a linear vehicle model is not always plausible.

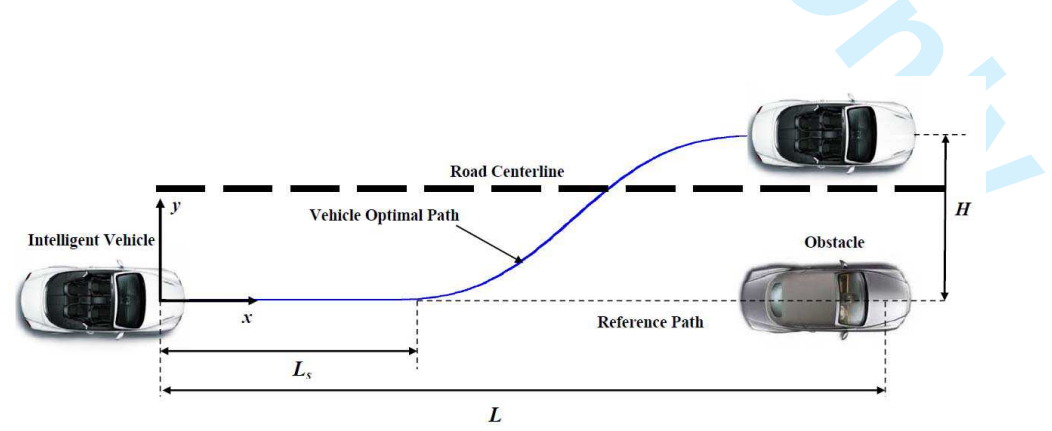

Figure 18. Path planning for collision avoidance relative to a moving obstacle (from Mashadi [91]). 


\section{Naturalistic Driving Control}

There is a possible need for automated driving to exhibit naturalistic properties, e.g. to be 'comfortable', 'smooth' and 'natural'. Certainly, drivers and occupants are aware of the actions of other vehicles and are sensitive to sudden changes in their behaviour. From an early driving study with ACC [18] it was reported that drivers are sensitive to small and sudden deceleration events when speed is automated - the tendency is to look forward to see the source of the event. Of course, for collision protection systems, there is no expectation to have naturalistic and smooth dynamic behaviour.

Ride comfort in road vehicles is normally associated with vertical road inputs in the form of road unevenness transmitted through the suspension [92]. Accelerations arising from cornering and braking are normally under the control of a human driver, and hence any resulting discomfort is not normally seen as part of the vehicle dynamics. But in cases where no human driver is immediately responsible for speed and/or steering control it may be that ride comfort will become a more important consideration in the longitudinal and lateral degrees of freedom. Existing experience from ACC and LKA should inform about the levels of acceleration and jerk that are normally acceptable, but it will be interesting to know whether passengers are indeed less forgiving of more fully automated driving systems.

If the design direction for automated driving systems is to emulate certain types of human driving, then there is much existing research to draw upon. The main characteristics of human driving were reviewed by MacAdam in [71]; there are many limitations in human drivers! Firstly, human driver suffer from time delays: reaction times for visualbased response are at least around $200 \mathrm{~ms}$, sometimes considerably more; auditory and tactile response times may be shorter, at around $140 \mathrm{~ms}$, though increasing as the stimulus reduces to near detection thresholds. Secondly there are visual and other sensitivity limits; according to several authors (see in [71]) driving is "90\% visual"; though vague, the statement broadly implies that, although other sources of information are relevant, the most critical information comes from human vision. For a "naturalistic driving machine" this does not of course imply using cameras and machine vision as the primary input for control - rather it emphasizes the need that humans have for preview information - to compensate for their inherent response delays and provide a safety margin for collision avoidance.

Adequate preview is also needed to achieve smooth steering control [71] irrespective of time delay. The dynamic response of human drivers can be characterised by bandwidth or, more specifically, by the crossover frequency. It has been shown that within some frequency range, the forward-path transfer function for steering approximates to the form $C(s) G(s)=\frac{\omega_{c} e^{-s T}}{s}$. Here the input is the error in lateral lane deviation and the output is vehicle lane position (in response to steering control from the driver). The crossover frequency is defined by the condition that the forward-path transfer function has unit gain; with the 'crossover model' form shown, the amplitude reduces at a rate of $20 \mathrm{~dB}$ per decade, helping to ensure the relative stability of the closed-loop system. A typical crossover frequency for an attentive driver is around $\omega_{c} \simeq 4$ radians/s, a little over $0.5 \mathrm{~Hz}$ [71]. One would expect that an acceptable lane keeping controller should have a similar frequency bandwidth, even though the stability margins can be greater, give a reduced time delay.

The literature has many examples which attempt to model human steering and speed control behaviour [71]. A common approach is to formulate a control model (for lateral or longitudinal control as appropriate) and perform parameter estimation to represent actual driving behaviour [90]. Commonly used control formulations are classical control 
[87], linear preview optimal control [93], [94] and Model Predictive Control [95], [96].

Data for parameter estimation and model validation is normally recorded from driving simulators or vehicle driving studies. More recently such research performed using data from naturalistic driving studies [97], [98]; in [98] a longitudinal driver model is formulated to include a range of noise signals and time delays designed to represent functional errors arising from the complexity of human behaviour - even though such complex behaviours are not directly represented in the model. One interesting aspect of this work is that the model can provide basic information about the effects of driver behaviour on crash outcomes; in [98] this was done using Monte-Carlo simulation, with random processes represented via a pseudo-random number generator.

A somewhat different approach is to look for detailed patterns in human driving, again as observed in naturalistic driving studies; here the drivers use instrumented vehicles to perform normal driving tasks, typically driving the same car for several weeks (or months or even longer). In [99] the authors propose a candidate error function that may be relevant to lateral control in highway driving, particularly for low-workload conditions and when there is no special reason for the driver to adhere to the lane center. This approach allows for driver models to be created based on real-world patterns of behaviour extracted from driving data. Validation is then needed, but it is a more stringent approach and has already yielded some interesting results. In [100] the authors determine that for steering control in lane-keeping, the driver tends to apply short correcting pulses that can be related to visually perceived tracking errors. A candidate model is presented and some validation and verification is made. However the authors show that there is much future work to be done in this area. Interestingly in [101] a similar pulse extraction is found, although the authors did not implement a computational model based on the findings.

As new generations of vehicles enjoy more sophisticated interfaces and control-level driver support (Section 3.1), there may be a need for further research into what becomes naturalistic with these new technical features.

\section{Automatic Control at the Limits of Friction}

In Section 6 the focus was on optimised and optimal automatic control for normal driving. Under more dynamic conditions, such as in collision avoidance, the need for optimal control is even more obvious - obtaining the best possible outcome given the limits of friction and available actuators. Vehicle control is challenging at the limits of handling but automated systems have the potential to safely negotiate difficult driving situations, where the human driver has neither the skills nor the same full range of actuators at his or her disposal.

As described previously, the architecture of Figure 9 shows the main elements of a 'typical' autonomous vehicle guidance system; in this approach an external control reference is used alongside a path-following algorithm, splitting the motion control into two parts [102].

In a similar but further developed approach for shared steering control, a mediator is included as an internal part of the control algorithm that explicitly considers vehicle stability and boundary constraints as it attempts to track a precomputed trajectory; such a mediator can choose to violate short term stability constraints in order to avoid a collision [103], [104] and [105]. In [105] experiments are conducted with X1, a rearwheel drive electric vehicle, shown in Figure 19. An integrated GPS-INS system provides vehicle states in real-time, and a ruggedized i7 computer outputs controller commands 
at $100 \mathrm{~Hz}$ to the vehicles drive-by-wire system.

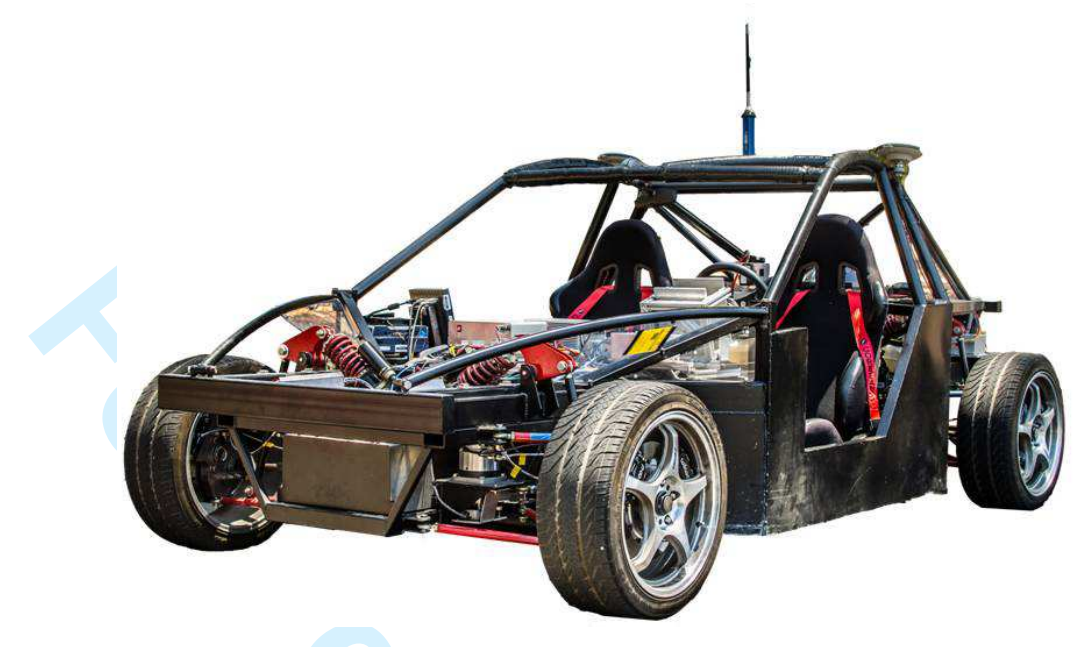

Figure 19. Steer-by-wire test vehicle X1 from Dynamic Design Lab, Stanford (from Funke [105]).

There is something of a conundrum regarding path planning (followed by path tracking). If a planned path is updated at each major computational time step, it cannot have any fundamental significance other than to simplify the control optimization problem to encode and represent the local constraints or objectives, for example to avoid collisions based on current vehicle states. On the other hand, if the planned path is not updated at each major time step then it surely has some fundamental significance - even given a set of possible initial vehicle states (including position) it is always the preferred path of motion for this initial set, even if it is not feasible! Except where there is a physical or other penalty for deviating from that path then surely this is incorrect - a more fundamental optimal control solution would not generate such a path at all. Of course it is common to make simplifying assumptions when solving complex problems, but especially when friction limits are approached, the wasteful nature of tracking a somewhat arbitrary reference path should be clear. And in either of the above cases the fundamental need for a pre-planned path is not at all clear.

In [106] a completely different approach was taken - a control strategy was developed around the pre-definition of a vector field: a spatially distributed set of target velocities defined in the plane of the road, this being used as a reference for desired CG motion. In Figure 20 such a field is shown in the form of its integral curves (the field is tangent to the flow lines shown). In this case there is no reference path, but there is a stable trajectory that a (particle) would follow, if for example the road looped around in a closed circuit. And another path (in this case the center-line) was used in the construction of the vector field (a previewed point on the centreline was used to define the direction of the field). The method was applied to a double lane-change manoeuvre [107] in which the vector field was pre-optimised for the road geometry (but not any vehicle states), and results compared well with a full application of general nonlinear optimal control methods (Pontryagin Minimum Principle).

Further recent work has shown that a path reference is unnecessary for friction-limited optimal control problems, e.g. for understeer compensation using independent four-wheel braking [108] and for path control during a potential multiple event accident using automated braking and steering [109]. In the first case a mass centre acceleration vector 
reference is used; in the second case the two-point boundary value problem was simplified using Quasi-Linear Optimal Control (QLOC), and it was found that optimal path control is feasible even while a high degree of yaw instability persists after an initial collision. Another example, where particle-based optimisation was used in preference to a target path, is in a collision avoidance scenario [110].

This is not to say that path planning is never necessary - for example in [111] a discrete optimization process is invoked to decide the optimum (discretised) path - but this type of problem is more relevant to robots moving in unstructured environments than to vehicles moving on highways. Some discrete decision making is necessary (and is not discussed here), and for low-speed manoeuvring it is perhaps always sensible to consider path planning as an important part of the optimal motion problem. But for friction-limited handling dynamics it appears there are often superior approaches available, for example to minimize off-tracking during understeer compensation, or minimizing expectation of harm during an automated safety-critical manoeuvre [112].

\section{Conclusion and Future Developments}

The motivation for this review paper was to understand new challenges for vehicle system dynamics in the era when there are unparalleled increases in the variety and level of automation in road vehicles, particularly the automation of driving itself. We have seen two perspectives in the march towards increasing automation: the 'ABS perspective' with automation gradually evolving on the vehicle; and the 'DARPA perspective' which employs large arrays of sensors, combined with artificial intelligence, to replace the human driver and replicate the widest possible range of human driving activities. The reality for highway vehicles is likely to be a combination of the two, building new 'intelligent 
vehicle' capabilities on a foundation of well-established vehicle dynamic control practice.

One clear idea emerging from the foregoing is the need to simplify the driving environment and hence reduce the tactical driving requirements for highly automated driving modes, e.g. Level 4 where the driver is not required to recover control in the event of some fault or disruption.

Another idea that emerges is that full automation (Level 5) implies a system that performs more like a personalized rapid transit system - i.e. more like a flexible rail transport system but without the physical rails. This seems to imply the introduction of a centralised command and control centre to operate and monitor the system.

Level 3 driving automation (the driver is required to recover control) requires a time window for the human to recover situation awareness and control capability, and again a structured and predictable environment is needed to guarantee reasonable time windows - at least 2-3 seconds, preferably longer. In fact the separation between Levels 3 and 4 hinges on whether the event "unresponsive driver" is handled deterministically by the system (level 4 has a fully operational fail-safe mode) or stochastically (Level 3 would employ a risk reduction technique such as gradual stopping with hazard lights showing).

All levels of driving automation benefit from collision protection, and lessons learned from supporting human drivers (Level $0+$ active safety) will naturally support the evolution of driving automation. And with Level 4 automation being seriously contemplated (no human needed for fail-safe operation) the need to handle on-board system failures (e.g. steering motor faulty) must be fully handled without using driver mechanical controls, at least during the available automated driving modes. Hence electrical and electronic system redundancy should be sufficient to handle such conditions, and in that case why insist on retaining mechanical connections to the driver at all? The same system-level redundancy for control can operate when there is a fault during manual driving; the need to retain a mechanical link between the driver and the road wheels (or brake cylinders) is no longer clear. Hence it might be that the push towards high levels of automation both enables and is enabled by a move toward a fully drive-by-wire vehicle design.

Another, somewhat surprising, conclusion from this review is that mapping and localization, conceptually very simple problems, remain a challenge for driving automation. In the absence of a 'magic fix' in the near future, the emphasis is likely to be on developing high levels of automation in limited geographical areas, where enhanced maps can be created and maintained in an adequate manner. Enhanced infrastructure ("smart roads") would then develop hand-in-hand with this, likely including highway and traffic monitoring to reduce risk and exclude unexpected hazards.

In summary, the grand challenges for artificial intelligence - entirely replacing the human driver - is likely too great to be solved in the next few decades, except where the immediate driving environment can be simplified. This may be a fully revamped infrastructure (in local regions) or it could be achieved by novel means (like the SARTRE road train concept). And the overriding challenge will be to ensure human safety for the new systems, knowing that most existing highway safety evaluation is based on retrospective studies.

\section{References}

[1] Rajamani R, Tan HS, Law BK, Zhang WB. Demonstration of integrated longitudinal and lateral control for the operation of automated vehicles in platoons. Control Systems Technology, IEEE Transactions on. 2000;8(4):695-708.

[2] Kiencke U, Nielsen L. Automotive Control Systems. Springer; 2005. 
[3] van Zanten A. Bosch ESP systems: 5 years of experience. SAE 2000-01-1633. 2000;.

[4] van Zanten A. Evolution of electronic control systems for improving the vehicle dynamic behavior. In: Proceedings of the 6th International Symposium on Advanced Vehicle Control; 2002. p. 1-9.

[5] Thrun S, Montemerlo M, Dahlkamp H, Stavens D, Aron A, Diebel J, Fong P, Gale J, Halpenny M, Hoffmann G, Lau K, Oakley C, Palatucci M, Pratt V, Stang P, Strohband S, Dupont C, Jendrossek LE, Koelen C, Markey C, Rummel C, Niekerk J, Jensen E, Alessandrini P, Bradski G, Davies B, Ettinger S, Kaehler A, Nefian A, Mahoney P. Stanley: The Robot That Won the DARPA Grand Challenge. The 2005 DARPA Grand Challenge, Springer Tracts in Advanced Robotics. 2007; $36: 1$.

[6] Buehler M, Iagnemma K, Singh S. The darpa urban challenge: autonomous vehicles in city traffic. Vol. 56. Springer Science \& Business Media; 2009.

[7] National Academy of Engineering. Frontiers of Engineering: Reports on Leading-Edge Engineering from the 2014 Symposium. Washington, DC: The National Academies Press; 2015.

[8] Michon JA. A critical view of driver behavior models: what do we know, what should we do? Springer; 1986.

[9] Shinar D, Tractinsky N, Compton R. Effects of practice, age, and task demands, on interference from a phone task while driving. Accident Analysis \& Prevention. 2005;37(2):315-326.

[10] Young K, Regan M, Hammer M. Driver distraction: A review of the literature. Distracted driving Sydney, NSW: Australasian College of Road Safety. 2007;:379-405.

[11] Wilson FA, Stimpson JP. Trends in fatalities from distracted driving in the united states, 1999 to 2008. American Journal of Public Health. 2010;100(11):2213-2219.

[12] Regan M, Young K. Use of Manual Speed Alerting and Cruise Control Devices by Drivers in New South Wales. Accident Research Centre, Monash University; 2004. Report no.:

[13] Vollrath M, Schleicher S, Gelau C. The influence of Cruise Control and Adaptive Cruise Control on driving behaviour-A driving simulator study. Accident Analysis \& Prevention. 2011;43(3).

[14] Fancher P, Bareket Z. Evaluating Headway Control Using Range Versus Range-Rate Relationships. Vehicle System Dynamics. 1994;23(1):575-596.

[15] Watanabe T, Kishimoto N, Hayafune K, Yamada K. Development of an Intelligent Cruise Control System. In: Proceedings of the World Congress on Intelligent Transport Systems; 1995. p. 12291235.

[16] Rajamani R. Vehicle Dynamics and Control. Springer; 2006.

[17] Mastinu G, Ploechl M, editors. Road and Off-Road Vehicle System Dynamics Handbook. CRC Press; 2014.

[18] Fancher P, Ervin R, Sayer J, Hagan M, Bogard S, Bareket Z, Mefford M, Haugen J. Intelligent cruise control field operational test. Final report. University of Michigan Transportation Research Institute Report; 1997. Report No.: UMTRI-97-11.

[19] Rakha H, Hankey J, Patterson A, Van Aerde M. Field Evaluation of Safety Impacts of Adaptive Cruise Control. ITS Journal. 2001;6:225-259.

[20] Inagaki T. Adaptive Automation: Sharing and Trading of Control; 2003. Chapter Chapter 8 of the Handbook of Cognitive Task Design; p. 147-169.

[21] Seppelt B, Lees M, Lee J. Driver Distraction and Reliance: Adaptive Cruise Control in the Context of Sensor Reliability and Algorithm Limits. In: Proceedings of the 3rd International Driving Symposium on Human Factors in Driver Assessment, Training, and Vehicle Design.; June 27-30. Rockport, Maine; 2005.

[22] Grover C, Knight I, Okoro F, Simmons I, Couper G, Massie P, Smith B. Automated Emergency Braking Systems: Technical requirements, costs and benefits. EC; 2008. Report no.:.

[23] Distner M, Bengtsson M, Broberg T, Jakobsson L. City Safety - A System Addressing Rear-End Collisions at Low Speeds. In: ESV 2009; 2009.

[24] Isaksson-Hellman I, Lindman M. The Effect of a Low-Speed Automatic Brake System Estimated From Real Life Data. In: ???; 2012.

[25] Ackermann J, Bunte T, Odenthal D. Advantages Of Active Steering For Vehicle Dynamics Control. $1999 ;$

[26] Chai Y, Kimura T, Igarashi K. Contribution for Vehicle Dynamics with a Steering System which Controls Tire Angles and Steering Force Independently. In: 12th International Symposium on Advanced Vehicle Control (AVED'14); September 22-26; 2014.

[27] Shibahata Y, Shimada K, Tomari T. Improvement of Vehicle Maneuverability by Direct Yaw Moment Control. Vehicle System Dynamics. 1993;

[28] Hancock MJ, Williams RA, Fina E, Best MC. Yaw motion control via active differentials. Trans Inst Meas Control. 2007 Jun;29(2):137-158. 
[29] Tomari T, Mori A, Shibahata Y. Development of SH-AWD Based on DYC (Direct Yaw Control) Concept. In: The 8th International Symposium on Advanced Vehicle Control; 2006. p. 611-616.

[30] Pohl J, Ekmark J. Development of a haptic intervention system for unintended lane departure. 2003;.

[31] Katzourakis D, Olsson C, Nedad L, Lidberg M. Driver Steering Override Strategies for Steering Based Active Safety Systems. In: FAST-zero 13, Second International Symposium on Future Active Safety Technology Toward zero traffic accidents. Nagoya, Japan; 2013.

[32] Gordon T, Sardar H, Blower D, Ljung Aust M, Bareket Z, Barnes M, Blankespoor A, IsakssonHellman I, Ivarsson J, Juhas B, et al. Advanced crash avoidance technologies (acat) program-final report of the volvo-ford-umtri project: safety impact methodology for lane departure warningmethod development and estimation of benefits; 2010. Report no.:

[33] Taxonomy and Definitions for Terms Related to On-Road Motor Vehicle Automated Driving Systems. 2014 January.

[34] Sharp RS, Peng H. Vehicle dynamics applications of optimal control theory. Vehicle System Dynamics. 2011;49(7):1073-1111.

[35] Tagesson K. Truck Steering System and Driver Interaction: Dissertation for Licentiate [dissertation]. Chalmers University of Technology; 2014.

[36] Pfeffer P. Interaction of Vehicle and Steering System Regarding On-Centre Handling [dissertation]. University of Bath; 2006.

[37] Ciarla V, Cahouet V, Canudas de Wit C, Quaine F. Genesis of booster curves in electric power assistance steering systems. In: The 15th International IEEE Conference on Intelligent Transportation Systems; 2012. p. 1345-1350.

[38] Klomp M. DRIVE MECHANISM FOR SELECTIVELY SWITCHING A DRIVE BETWEEN PROPULSION AND TORQUE VECTORING. 2011.

[39] Stanton NA, Marsden P. From fly-by-wire to drive-by-wire: Safety implications of automation in vehicles. Safety Science. 1996;24(1):35 - 49.

[40] Shladover S, Desoer C, Hedrick K, Tomizuka M, Walrand J, Zhang WB, McMahon D, Peng H, Sheikholeslam S, McKeown N. Automatic Vehicle Control Developments in the PATH Program. IEEE Transactions on Vehicular Technology. 1991 February;40(1):114-130.

[41] Bergenhem C, Huang Q, Benmimoun A, Robinson T. Challenges of Platooning on Public Motorways. In: 17th World Congress and Exhibition on Intelligent Transport Systems and Services (ITS 2010); 2010. p. 1-12.

[42] Dodson S. Welcome to the transport of tomorrow. The Guardian. 2007 October 10;.

[43] Why Has Masdar Personal Rapid Transit (PRT) Been Scaled Back? In: Conference on Personal Rapid Transit; September 21-23rd; 2010.

[44] Vincent J. Milton keynes introducing driverless public transport pods by 2017. The Independent. 2013 November 6;.

[45] Shladover SE, et al. Lane assist systems for bus rapid transit, volume i: Technology assessment. Publication RTA 65A0160, US Department of Transportation, Washington, DC. 2007; .

[46] Falcone P, Borrelli F, Tseng E, Hrovat D. On Low Complexity Predictive Approaches to Control of Autonomous Vehicles. Springer-Verlag; 2010. Chapter Chapter 13; p. 195-210.

[47] Tsang SH, Hall PS, Hoare EG, Clarke NJ. Advance path measurement for automotive radar applications. Intelligent Transportation Systems, IEEE Transactions on. 2006;7(3):273-281.

[48] Leonard J, Barrett D, How J, Teller S, Antone M, Campbell S, Epstein A, Fiore G, Fletcher L, Frazzoli E, et al. Team mit urban challenge technical report. 2007;

[49] Mei C, Sibley G, Cummins M, Newman P, Reid I. Rslam: A system for large-scale mapping in constant-time using stereo. International journal of computer vision. 2011;94(2):198-214.

[50] Urmson C, Ragusa C, Ray D, Anhalt J, Bartz D, Galatali T, Gutierrez A, Johnston J, Harbaugh S, Messner W, et al. A robust approach to high-speed navigation for unrehearsed desert terrain. Journal of Field Robotics. 2006;23(8):467-508.

[51] Toledo-Moreo R, Bétaille D, Peyret F. Lane-level integrity provision for navigation and map matching with gnss, dead reckoning, and enhanced maps. Intelligent Transportation Systems, IEEE Transactions on. 2010;11(1):100-112.

[52] Joshi A, James M. Generation of accurate lane-level maps from coarse prior maps and lidar. Intelligent Transportation Systems Magazine, IEEE. 2015;7(1):19-29.

[53] Haklay M, Weber P. Openstreetmap: User-generated street maps. Pervasive Computing, IEEE. $2008 ; 7(4): 12-18$.

[54] Graefe G, Caspary W, Heister H, Klemm J, Sever M. The road data acquisition system mosesdetermination and accuracy of trajectory data gained with the applanix pos/lv. In: Proceedings, 
The Third International Mobile Mapping Symposium, Cairo, Egypt, January. Citeseer; 2001. p. $3-5$.

[55] Krygier J, Wood D. Making maps, second edition: A visual guide to map design for gis. Guilford Publications; 2011; Available from: https://books.google.co.uk/books?id=jr2fZEitOnkC.

[56] Cheng W, Cheng X, Song M, Chen B, Zhao WW. On the design and deployment of rfid assisted navigation systems for vanets. Parallel and Distributed Systems, IEEE Transactions on. 2012; 23(7):1267-1274.

[57] Misener JA, Sengupta R, Ahern K, Datta Gupta S, Dickey S, Kuhn T, Lian T, Manasseh C, Nelson D, Rezai S, et al. Vii california: Development and deployment proof of concept and groupenabled mobility and safety (gems); 2010. Report no.:.

[58] Alam N, Balaei AT, Dempster AG. An instantaneous lane-level positioning using dsrc carrier frequency offset. Intelligent Transportation Systems, IEEE Transactions on. 2012;13(4):1566-1575.

[59] Shladover S. Review of the state of the development of development of advanced vehicle control systems (AVCS). Vehicle System Dynamics. 1995;(24):551-595.

[60] Distner M, Bengtsson M, Broberg T, Jakobsson L. City safetya system addressing rear-end collisions at low speeds. In: Proc. 21st International Technical Conference on the Enhanced Safety of Vehicles; 09-0371; 2009.

[61] Hamdane H, Serre T, Anderson R, Masson C, Yerpez J. Description of pedestrian crashes in accordance with characteristics of active safety systems. In: IRCOBI Conference Proceedings; 2014.

[62] Coelingh E, Eidehall A, Bengtsson M. Collision warning with full auto brake and pedestrian detection-a practical example of automatic emergency braking. In: Intelligent Transportation Systems (ITSC), 2010 13th International IEEE Conference on. IEEE; 2010. p. 155-160.

[63] Chun J, Lee I, Park G, Seo J, Choi S, Han SH. Efficacy of haptic blind spot warnings applied through a steering wheel or a seatbelt. Transportation Research Part F: Traffic Psychology and Behaviour. 2013;21:231-241.

[64] Glazduri V. An investigation of the potential safety benefits of vehicle backup proximity sensors. In: Proceedings of the International Technical Conference on Enhanced Safety Vehicles; Vol. 1; 2005. p. 2.

[65] Habibovic A. Analyzing real-world data to promote development of active safety systems that reduce car-to-vulnerable road user accidents. Chalmers University of Technology; 2012.

[66] Dept of Transportation (US) NHTSAN. Traffic Safety Facts 2012.; 2012. Report no.:.

[67] Da Lio M, Biral F, Bertolazzi E, Galvani M, Bosetti P, Windridge D, Saroldi A, Tango F. Artificial co-drivers as a universal enabling technology for future intelligent vehicles and transportation systems. IEEE Transactions on Intelligent Transportation Systems. 2015;16(1):244-263.

[68] Flemisch F, Nashashibi F, Rauch N, Schieben A, Glaser S, Temme G, Resende P, Vanholme B, Löper C, Thomaidis G, et al. Towards highly automated driving: Intermediate report on the haveitjoint system. In: 3rd European Road Transport Research Arena, TRA 2010; 2010.

[69] Rizzi M, Kullgren A, Tingvall C. The injury crash reduction of low-speed autonomous emergency braking (aeb) on passenger cars. In: IRCOBI Conference Proceedings; 2014.

[70] More good news about crash avoidance: Volvo city safety reduces crashes. ????;

[71] Macadam CC. Understanding and modeling the human driver. Vehicle System Dynamics. 2003; 40(1-3):101-134.

[72] Li SE, Peng H, Li K, Wang J. Minimum fuel control strategy in automated car-following scenarios. Vehicular Technology, IEEE Transactions on. 2012;61(3):998-1007.

[73] Suthaputchakun C, Sun Z, Dianati M. Applications of vehicular communications for reducing fuel consumption and co 2 emission: the state of the art and research challenges. Communications Magazine, IEEE. 2012;50(12):108-115.

[74] Guzolek J, Koch E. Real-time route planning in road networks. In: Vehicle Navigation and Information Systems Conference, 1989. Conference Record. IEEE; 1989. p. 165-169.

[75] Brockfeld E, Barlovic R, Schadschneider A, Schreckenberg M. Optimizing traffic lights in a cellular automaton model for city traffic. Physical Review E. 2001;64(5):056132.

[76] Xu Y, Zhang Y, Liu M. Multiagent based decentralized traffic light control for large urban transportation system. Mathematical Problems in Engineering. 2014;2014.

[77] Garcia-Nieto J, Olivera AC, Alba E. Optimal cycle program of traffic lights with particle swarm optimization. Evolutionary Computation, IEEE Transactions on. 2013;17(6):823-839.

[78] Ioannou P, Wang Y, Chang H. Integrated roadway/adaptive cruise control system: Safety, performance, environmental and near term deployment considerations. California PATH Program, Institute of Transportation Studies, University of California at Berkeley; 2007.

[79] Reghelin R, Arruda L. Optimizing travel time of intelligent vehicles in any highway configuration. 
Advances in Transportation Studies. 2014;(32).

[80] Pérez J, Milanés V, Godoy J, Villagra J, Onieva E. Cooperative controllers for highways based on human experience. Expert Systems with Applications. 2013;40(4):1024-1033.

[81] Milanés V, Shladover SE, Spring J, Nowakowski C, Kawazoe H, Nakamura M. Cooperative adaptive cruise control in real traffic situations. Intelligent Transportation Systems, IEEE Transactions on. 2014;15(1):296-305.

[82] US Department of Transportation. Recent International Activity in Cooperative VehicleHighway Automation Systems. 2012;.

[83] Chan E. Sartre automated platooning vehicles. In: Transport Research Arena (TRA) 5th Conference: Transport Solutions from Research to Deployment; 2014.

[84] Kim TS, Manzie C, Sharma R. Model predictive control of velocity and torque split in a parallel hybrid vehicle. In: Systems, Man and Cybernetics, 2009. SMC 2009. IEEE International Conference on. IEEE; 2009. p. 2014-2019.

[85] Johannesson L, Egardt B. A novel algorithm for predictive control of parallel hybrid powertrains based on dynamic programming. In: Advances in Automotive Control; Vol. 5; 2007. p. 343-350.

[86] Ferreira M, Fernandes R, Conceição H, Viriyasitavat W, Tonguz OK. Self-organized traffic control. In: Proceedings of the seventh ACM international workshop on VehiculAr InterNETworking. ACM; 2010. p. 85-90.

[87] Weir DH, McRuer DT. Dynamics of driver vehicle steering control. Automatica. 1970;6(1):87-98.

[88] Lu G, Tomizuka M. Vehicle following as backup control schemes for magnet-magnetometer-based lateral guidance. Control Systems Technology, IEEE Transactions on. 2005;13(2):274-285.

[89] Guo K, Guan H. Modelling of driver/vehicle directional control system. Vehicle System Dynamics. 1993;22(3-4):141-184.

[90] Plöchl M, Edelmann J. Driver models in automobile dynamics application. Vehicle System Dynamics. 2007;45(7-8):699-741.

[91] Mashadi B, Majidi M. Global optimal path planning of an autonomous vehicle for overtaking a moving obstacle. Latin American Journal of Solids and Structures. 2014;11(14):2555-2572.

[92] Griffin MJ. Discomfort from feeling vehicle vibration. Vehicle System Dynamics. 2007;45(7-8):679698.

[93] MacAdam CC. An optimal preview control for linear systems. Journal of dynamic systems, measurement, and control. 1980;102(3):188-190.

[94] Odhams A, Cole D. Application of linear preview control to modelling human steering control. Proceedings of the Institution of Mechanical Engineers, Part D: Journal of Automobile Engineering. 2009;223(7):835-853.

[95] Prokop G. Modeling human vehicle driving by model predictive online optimization. Vehicle System Dynamics. 2001;35(1):19-53.

[96] Keen SD, Cole DJ. Steering control using model predictive control and multiple internal models. In: Proceedings of the 8th International Symposium on Automotive Control (AVEC 2006); 2006. p. 599-604.

[97] Lee K, Peng H. Identification and verification of a longitudinal human driving model for collision warning and avoidance systems. International journal of vehicle autonomous systems. 2004;2(1):317.

[98] Yang HH, Peng H. Development and evaluation of collision warning/collision avoidance algorithms using an errable driver model. Vehicle System Dynamics. 2010;48(S1):525-535.

[99] Gordon T, Blankespoor A, Barnes B, Blower D, Green P, Kostyniuk L. Yaw rate error-a dynamic measure of lane keeping control performance for the retrospective analysis of naturalistic driving data. In: 21st International Technical Conference on the Enhanced Safety of Vehicles, Stuttgart, Germany; 2009. p. 09-0326.

[100] Gordon T, Srinivasan K. Modeling human lane keeping control in highway driving with validation by naturalistic data. In: Systems, Man and Cybernetics (SMC), 2014 IEEE International Conference on. IEEE; 2014. p. 2507-2512.

[101] Benderius O, Markkula G. Evidence for a fundamental property of steering. In: Proceedings of the Human Factors and Ergonomics Society Annual Meeting; Vol. 58. SAGE Publications; 2014. p. 884-888.

[102] Falcone P, Borrelli F, Asgari J, Tseng H, Hrovat D. Predictive Active Steering Control for Autonomous Vehicle Systems. IEEE Transactions on Control Systems Technology. 2007 May; 15(3):566-579.

[103] Beal C. Applications of Model Predictive Control to Vehicle Dynamics for Active Safety and Stability [dissertation]. Stanford University; 2011. 
[104] Erlien S, Susumu F, Gerdes C. Safe Driving Envelopes for Shared Control of Ground Vehicles. In: 7th IFAC Symposium on Advances in Automotive Control; 2010.

[105] Funke J, Brown M, Erlien S, Gerdes J. Prioritizing Collision Avoidance and Vehicle Stabilization for Autonomous Vehicles. To be submitted. 2015;.

[106] Gordon T, Best MC, Dixon P. An automated driver based on convergent vector fields. Proceedings of the Institution of Mechanical Engineers, Part D: Journal of Automobile Engineering. 2002; 216(4):329-347.

[107] Gordon T, Best MC. On the synthesis of driver inputs for the simulation of closed-loop handling manoeuvres. International Journal of Vehicle Design. 2006;40(1):52-76.

[108] Klomp M, Lidberg M, Gordon T. On optimal recovery from terminal understeer. Proceedings of the Institution of Mechanical Engineers, Part D: Journal of Automobile Engineering. 2014;.

[109] Yang D, Gordon TJ, Jacobson B, Jonasson M. Quasi-Linear Optimal Path Controller Applied to Post Impact Vehicle Dynamics. submitted for publication in IEEE Transactions on Intelligent Transportation Systems. 2011; .

[110] Moshchuk N, Chen SK, Zagorski C, Chatterjee A. Optimal braking and steering control for active safety. In: Intelligent Transportation Systems (ITSC), 2012 15th International IEEE Conference on. IEEE; 2012. p. 1741-1746.

[111] Rashid AT, Ali AA, Frasca M, Fortuna L. Path planning with obstacle avoidance based on visibility binary tree algorithm. Robotics and Autonomous Systems. 2013;61(12):1440-1449.

[112] Yang D. Vehicle dynamics control after impacts in multiple-event accidents: doctoral dissertation. Chalmers University of Technology; 2013. 